\title{
ROUGHNESS SENSITIVITY COMPARISONS \\ OF WIND TURBINE BLADE SECTIONS
}

\author{
A Dissertation \\ by \\ BENJAMIN JACOB WILCOX
}

\begin{abstract}
Submitted to the Office of Graduate and Professional Studies of Texas A\&M University

in partial fulfillment of the requirements for the degree of

DOCTOR OF PHILOSOPHY
\end{abstract}

\begin{abstract}
Chair of Committee, Edward White
Committee Members, Moble Benedict

Diego Donzis

Partha Mukherjee

Head of Department, Rodney Bowersox
\end{abstract}

December 2016

Major Subject: Aerospace Engineering

Copyright 2016 Benjamin Jacob Wilcox 


\begin{abstract}
One explanation for wind turbine power degradation is insect roughness. Historical studies on insect-induced power degradation have used simulation methods which are either unrepresentative of actual insect roughness or too costly or time-consuming to be applied to wide-scale testing. Furthermore, the role of airfoil geometry in determining the relations between insect impingement locations and roughness sensitivity has not been studied.

To link the effects of airfoil geometry, insect impingement locations, and roughness sensitivity, a simulation code was written to determine representative insect collection patterns for different airfoil shapes. Insect collection pattern data was then used to simulate roughness on an NREL S814 airfoil that was tested in a wind tunnel at Reynolds numbers between $1.6 \times 10^{6}$ and $4.0 \times 10^{6}$. Results are compared to previous tests of a NACA $63_{3}-418$ airfoil.

Increasing roughness height and density results in decreased maximum lift, lift curve slope, and lift-to-drag ratio. Increasing roughness height, density, or Reynolds number results in earlier bypass transition, with critical roughness Reynolds numbers lying within the historical range. Increased roughness sensitivity on the $25 \%$ thick NREL S814 is observed compared to the $18 \%$ thick NACA $63_{3}-418$.

Blade-element-momentum analysis was used to calculate annual energy production losses of $4.9 \%$ and $6.8 \%$ for a NACA $63_{3}-418$ turbine and an NREL S814 turbine, respectively, operating with $200 \mu \mathrm{m}$ roughness. These compare well to historical field measurements.
\end{abstract}


To my family,

for years of loving support. 


\section{ACKNOWLEDGEMENTS}

I would first like to thank Sandia National Laboratories under contract number 1209202 with David Maniaci as technical monitor for providing the funds and resources to complete this project. I would also like to acknowledge Chris Langel and Dr. C.P. van Dam whose computational work on the project was invaluable.

This work would not have been possible without years of support from countless individuals at Texas A\&M. The employees of the Oran W. Nicks Low Speed Wind Tunnel deserve special recognition: Zahir Udovicic for a beautiful wind tunnel model; Ric Warren and Jon Kochan for leading model installation and tunnel operations; and Doug Kutz, John Stanford, and Lisa Brown for providing tunnel control and data analysis. I'd also like to thank my coworkers at the Klebanoff-Saric Wind Tunnel: Jason Monshke and Matt Kuester for teaching me how to be an experimentalist; Jeffrey Lestico, Krista Cratty, Alex Berger, and Robert Long for their help in conducting the tests and analyzing results; and Robert Ehrmann for laying the foundations for the project and guiding me in its early stages. Finally, I'd like to acknowledge my committee members for their insight and especially my advisor, Dr. Edward White, for molding me into the researcher that I am today.

I also owe great thanks to my friends and family members: to my parents who have loved and encouraged me in all my endeavors; to my sisters who have been role models for me since day one; to my brother who has always been there when I needed him; to my friends who have shown me the beauty and joy in the world; and lastly to my Lord and Savior, Jesus Christ, whose love sustains me. 


\section{TABLE OF CONTENTS}

Page

ABSTRACT ................................

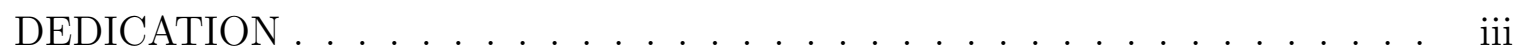

ACKNOWLEDGEMENTS ............................ iv

TABLE OF CONTENTS . . . . . . . . . . . . . . . . . . v v

LIST OF FIGURES . . . . . . . . . . . . . . . . . . . . vii

LIST OF TABLES ........................ . . xi

CHAPTER I: INTRODUCTION . . . . . . . . . . . . . . . . 1

I.A. Turbine Control Schemes . . . . . . . . . . . . 2

I.B. Insect-Induced Performance Degradation . . . . . . . . 5

I.C. Historical Airfoil Design . . . . . . . . . . . . . 9

I.D. Roughness Effects on Boundary-Layer Transition . . . 12

I.E. Insect Impingement Modeling . . . . . . . . . . . . . . 15

I.F. Research Objectives . . . . . . . . . . . . . . 17

CHAPTER II: INSECT IMPINGEMENT ANALYSIS . . . . . . . . . . . . . . 19

II.A. Trajectory Modeling . . . . . . . . . . . . . . . . . 19

II.B. Insect Population Modeling . . . . . . . . . . . . . . 23

II.C. Blade Modeling . . . . . . . . . . . . . . . . . . . 24

II.D. Insect Collection Parameters . . . . . . . . . . . . 24

II.E. Airfoil Selection . . . . . . . . . . . . . . . 27

II.F. Insect Distribution Results . . . . . . . . . . . . . 31

CHAPTER III: WIND TUNNEL TESTING . . . . . . . . . . . . . . . . 37

III.A. Wind Tunnel Facilities . . . . . . . . . . . . . . . 37

III.B. Model Construction . . . . . . . . . . . . . . . . 42

III.C. Roughness Modeling . . . . . . . . . . . . . . 45

III.D. Testing Procedure . . . . . . . . . . . . . . . . 49 
III.E. Test Instrumentation . . . . . . . . . . . . . . 50

III.F. Lift, Pitching Moment, and Drag Measurements . . . . 52

III.G. Wind Tunnel Corrections . . . . . . . . . . . . 57

III.H. Transition Location Measurements . . . . . . . . . . 60

III.I. Lift, Drag, and Pitching Moment Results . . . . . . . 68

III.J. Transition Location Results . . . . . . . . . . . . . 78

CHAPTER IV: ANNUAL ENERGY PRODUCTION ANALYSIS . . . . . . . 89

IV.A. Blade-Element-Momentum Theory . . . . . . . . . . 89

IV.B. Blade-Element-Momentum Corrections . . . . . . . . . 93

IV.C. Blade-Element-Momentum Simulation . . . . . . . . 95

IV.D. Annual Energy Production Calculations . . . . . . . . 98

IV.E. Annual Energy Production Results . . . . . . . . . . . 99

CHAPTER V: CONCLUSIONS \& FUTURE WORK . . . . . . . . . . . . . . 104

REFERENCES . . . . . . . . . . . . . . . . . . 108

APPENDIX A: AIRFOIL COORDINATES . . . . . . . . . . . . . . 115

APPENDIX B: FLOW UNIFORMITY SUMMARY . . . . . . . . . . . . . 116

APPENDIX C: PRESSURE TAP COORDINATES . . . . . . . . . . . . . 120

APPENDIX D: LIFT, PITCHING MOMENT, AND DRAG SUMMARY … 121

APPENDIX E: PERFORMANCE LOSS SUMMARY … . . . . . . . . . . 129

APPENDIX F: TRANSITION LOCATION SUMMARY . . . . . . . . . . 132

APPENDIX G: CRITICAL REYNOLDS NUMBER SUMMARY . . . . . . . . 136

APPENDIX H: TURBINE SIMULATION GEOMETRY . . . . . . . . . . 138 


\section{LIST OF FIGURES}

FIGURE

Page

I.1 Example turbine power curve. . . . . . . . . . . . . . . . . 3

I.2 Example insect roughness images. . . . . . . . . . . . . . . . . . 5

II.1 Example insect trajectory plots. . . . . . . . . . . . . . . . . . . 22

II.2 Collection efficiency definition diagram. . . . . . . . . . . . 26

II.3 Wrap distance definition diagram. . . . . . . . . . . . . . . . . . 26

II.4 Airfoil collection efficiency comparisons. . . . . . . . . . . . . . . . 29

II.5 Airfoil pressure coefficient comparisons. . . . . . . . . . . . . . 30

II.6 Airfoil profiles. . . . . . . . . . . . . . . . . . . . . . . 31

II.7 NACA $63_{3}-418$ and NREL S814 collection efficiency diagrams. . . . . 32

II.8 Collection efficiency and impact velocity comparison. . . . . . . . . . 33

II.9 Impact velocity distribution for the NACA $63_{3}-418$ turbine. . . . . . 35

II.10 Impact velocity distribution for the NREL S814 turbine. . . . . . . . 35

II.11 Collection efficiency distribution for the NACA $63_{3}-418$ turbine. . . . 36

II.12 Collection efficiency distribution for the NREL S814 turbine. . . . . . 36

III.1 Drawing of wind tunnel test section. . . . . . . . . . . . . . . . . . . 39

III.2 Hotwire temperature compensation plots. . . . . . . . . . . . . . 41

III.3 Hotwire calibration plots. . . . . . . . . . . . . . . . . . . . . 42

III.4 Flow uniformity plot at $R e_{c}=3.2 \times 10^{6} \ldots \ldots \ldots$. . . . . . 43

III.5 Drawing of NREL S814 wind tunnel model. . . . . . . . . . . . . . . 44

III.6 Roughness region comparison. . . . . . . . . . . . . . . . . 47 
III.7 Insect roughness patterns. . . . . . . . . . . . . . . . . . . . . . . . 48

III.8 Example pressure coefficient plots. . . . . . . . . . . . . . . . . 53

III.9 Example wake plots. . . . . . . . . . . . . . . . . . . . 55

III.10 Example infrared images. . . . . . . . . . . . . . . . . . . . 62

III.11 Example pixel intensity plots. . . . . . . . . . . . . . . . . . . 64

III.12 Example transition detection algorithm output image. . . . . . . . . 65

III.13 $R e_{k}$ variation with surface location plot. . . . . . . . . . . . 66

III.14 $R e_{k, \max }$ variation with angle of attack plot. . . . . . . . . 67

III.15 Lift variation with angle of attack for varying roughness height and density on the NACA $63_{3}-418$ at $R e_{c}=3.2 \times 10^{6}$. . . . . . . 69

III.16 Pitching moment variation with angle of attack for varying roughness height and density on the NACA $63_{3}-418$ at $R e_{c}=$ $3.2 \times 10^{6} \ldots \ldots \ldots \ldots \ldots 70 \ldots \ldots$. . . . . . . . . . . . . . . . . . . .

III.17 Drag variation with angle of attack for varying roughness height and density on the NACA $63_{3}-418$ at $R e_{c}=3.2 \times 10^{6} \ldots$. . . 70

III.18 Lift variation with angle of attack for varying roughness height and density on the NREL S814 at $R e_{c}=3.2 \times 10^{6}$. . . . . . . . . 73

III.19 Pitching moment variation with angle of attack for varying roughness height and density on the NREL S814 at $R e_{c}=3.2 \times 10^{6} . \quad 74$

III.20 Drag variation with angle of attack for varying roughness height and density on the NREL S814 at $R e_{c}=3.2 \times 10^{6}$. . . . . . 74

III.21 Lift variation with angle of attack (zoomed version) for varying roughness height and density on the NREL S814 at $R e_{c}=3.2 \times 10^{6} .75$ 
III.22 Pitching moment variation with angle of attack (zoomed version) for varying roughness height and density on the NREL S814 at $R e_{c}=3.2 \times 10^{6}$. . . . . . . . . . . . . . . . . . 75

III.23 Lift variation with angle of attack for varying Reynolds number on the NREL S814 clean configuration. . . . . . . . . . . . . . . 79

III.24 Drag variation with angle of attack for varying Reynolds number on the NREL S814 clean configuration. . . . . . . . . . . . . . . 79

III.25 Lift variation with angle of attack for varying Reynolds number on the NREL S814 $100 \mu \mathrm{m} 03 \%$ configuration. . . . . . . . . . . 80

III.26 Drag variation with angle of attack for varying Reynolds number on the NREL S814 $100 \mu \mathrm{m} 03 \%$ configuration. . . . . . . . . . . 80

III.27 Upper surface transition location variation with angle of attack for varying roughness height and density on the NACA $63_{3^{-}}$ 418 at $R e_{c}=3.2 \times 10^{6}$. . . . . . . . . . . . . . . . . . . . 82

III.28 Upper surface transition location variation with angle of attack for varying roughness height and density on the NREL S814 at $R e_{c}=3.2 \times 10^{6}$. . . . . . . . . . . . . . . . . . . . 84

III.29 Lower surface transition location variation with angle of attack for varying roughness height and density on the NREL S814 at $R e_{c}=3.2 \times 10^{6} \ldots \ldots \ldots$. . . . . . . . . . . . . . 84

III.30 Upper surface transition location variation with angle of attack for varying Reynolds number on the NREL S814 clean configuration. ................. 8

III.31 Lower surface transition location variation with angle of attack for varying Reynolds number on the NREL S814 clean configuration. . . . . . . . . . . . . . . 87

III.32 Upper surface transition location variation with angle of attack for varying Reynolds number on the NREL S814 $100 \mu \mathrm{m} \mathrm{03 \%}$ configuration. . . . . . . . . . . . . . . . . . 88 
FIGURE

III.33 Lower surface transition location variation with angle of attack for varying Reynolds number on the NREL S814 $100 \mu \mathrm{m} \mathrm{03 \%}$

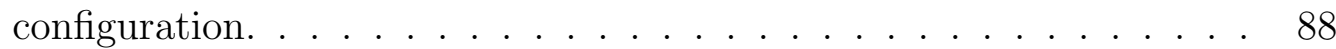

IV.1 Momentum theory control volume. . . . . . . . . . . . . . . . 90

IV.2 Turbine section velocity and force components. . . . . . . . . . . . 92

IV.3 Example $360^{\circ}$ lift and drag polars. . . . . . . . . . . . . . 97

IV.4 Map of U.S. wind resource. . . . . . . . . . . . . . . . . . 99

IV.5 Power degradation plots. . . . . . . . . . . . . . . . 100

IV.6 Rayleigh wind distribution plots with clean power curve overlay. . . . 101 


\section{LIST OF TABLES}

TABLE

Page

III.1 Performance losses on the NACA $63_{3}-418$ at $R e_{c}=3.2 \times 10^{6} . \quad \ldots \quad 72$

III.2 Performance losses on the NREL S814 at $R e_{c}=3.2 \times 10^{6} \ldots \ldots 77$

III.3 Critical roughness Reynolds numbers on the NACA $63_{3}-418$ upper surface at $R e_{c}=3.2 \times 10^{6}$. . . . . . . . . . . . . . 83

III.4 Critical roughness Reynolds numbers on the NREL S814 upper surface at $R e_{c}=3.2 \times 10^{6}$. . . . . . . . . . . . . . . . . . 85

III.5 Critical roughness Reynolds numbers on the NREL S814 lower surface at $R e_{c}=3.2 \times 10^{6} \ldots \ldots$. . . . . . . . . . . . . . . 85

IV.1 AEP losses due to roughness on the NACA $63_{3}-418$ turbine. . . . . . 103

IV.2 AEP losses due to roughness on the NREL S814 turbine. . . . . . . 103

IV.3 AEP losses due to roughness on the combined NACA $63_{3}-418$ and NREL S814 turbine. . . . . . . . . . . . . . . . . . 103 


\section{CHAPTER I}

\section{INTRODUCTION}

Wind farm operators routinely cite lower-than-predicted energy production. Much of this problem stems from an inadequate understanding of the wind resource. Boccard found that capacity factors, ratios of average turbine power to nameplate power, have been overestimated by as much as $66 \%$ in Europe during the past decade [1]. Changes in annual wind potential, non-optimal farm siting, and lower-than-expected turbine availability all contribute to this issue. An aerodynamic explanation for the discrepancy is differences between actual and expected power curves of turbines. Power curves, graphs of turbine power output versus wind speed, are used by turbine manufacturers to guarantee the performance of their products to wind farm developers and financiers. These curves are often based on performance measurements of turbines which may not be typical of actual field performance [2]. Accurate power curve predictions are essential to wind farm developers and operators who use these curves to forecast power performance.

A common cause of power curve degradation is blade roughness due to erosion, paint chipping, or insect deposits. Each harms blade performance by decreasing the maximum lift and lift-curve slope, and by increasing drag [3]. Various techniques have been used to simulate blade roughness both experimentally and computationally, but accurately quantifying these effects has proved difficult. A particularly troublesome difficulty is the significant variability in location and distribution of roughness on different blades. These factors contribute to the overall amount of power degradation that occurs, yet few studies exist which examine them. This dissertation aims to 
correct this by providing a set of tools which can be used by blade designers to generate expected roughness locations for insect roughness. Additionally, wind tunnel tests for two turbine airfoils and a variety of realistic insect roughness configurations were conducted. These are used to quantify the effects of airfoil shape and roughness severity on turbine power and annual energy production (AEP).

\section{I.A. Turbine Control Schemes}

Turbines were historically designed to maintain rated power through stalling of outboard blade sections. For a constant rotor rotation speed, the apparent angle of attack of the blades increases as wind velocity increases. At high wind velocities, this leads to stalling of the blade tip which moves inboard as velocity is further increased. The main benefit of this control scheme is its mechanical simplicity which led it to be utilized as the form of power control on nearly all early turbines. In contrast, modern turbines usually control power through pitch-control mechanisms which actively decreases the blade's angle of attack once rated power is reached. Typical control mechanisms consist of hydraulic systems which are connected to an electronic power output sensor, although control using stepped electrical motors is becoming common. The pitch-control scheme sacrifices mechanical simplicity for aerodynamic efficiency and the ability to maintain rated power nearly exactly once wind speeds are sufficient. A final control scheme called active-stall exists which is a combination of pitch-control and stall-control. Instead of decreasing angle of attack to limit maintain rated power, active-stall turbines increase angle of attack to induce stall, but their operation is otherwise identical to pitch-control machines. 
Turbines also include constant-speed and variable-speed operating modes. For constant-speed turbines, the rotor rotational speed is constant and often selected to match the frequency of the power grid in which it operates. This reduces electrical complexity but does not allow the turbine to operate at its most aerodynamically efficient rotational speed. Variable-speed rotors control rotational speed by varying the generator torque demanded from the rotor. This allows the rotor to operate at its optimal speed but requires additional sensing and frequency conversion mechanisms. Pitch control and speed control are independent, so any combination of pitch and speed control is possible.

Typical wind turbine power curves such as that shown in Figure I.1 are separated into four regions. Region I contains all wind speeds below the cut-in wind speed, the speed necessary for startup to begin. Turbines are not operated in Region I because generator and drivetrain losses prevent the turbine from producing power in this region.

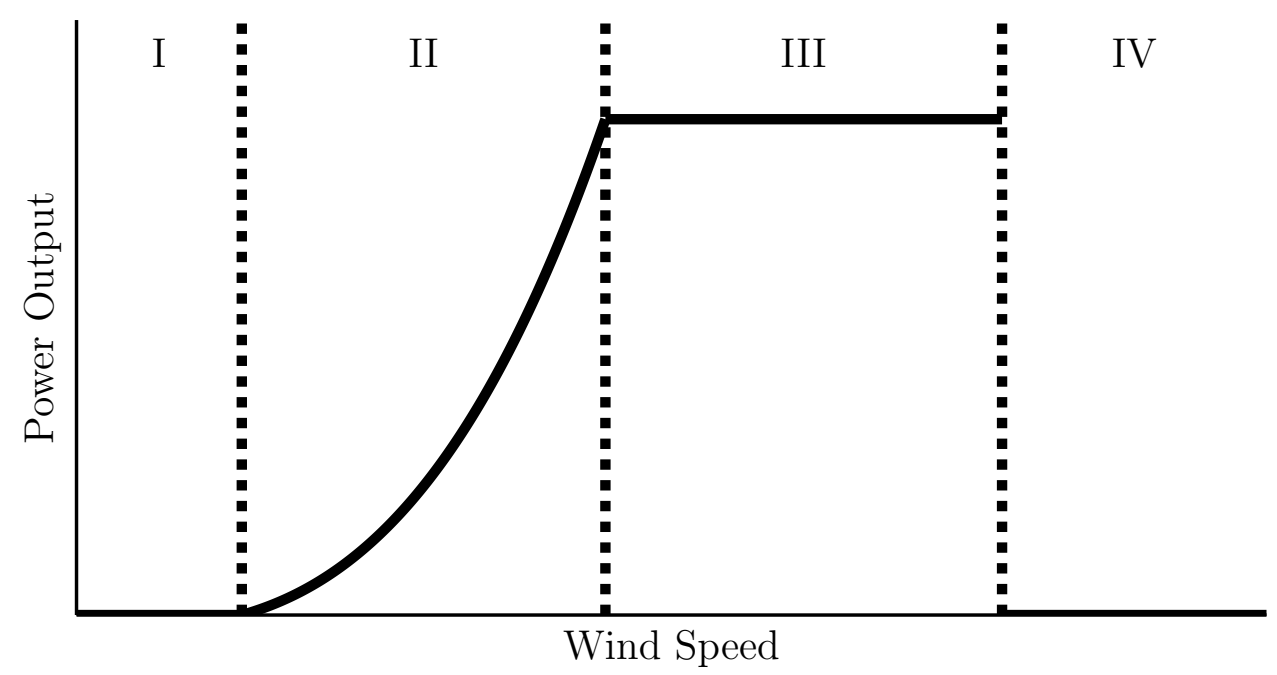

Figure I.1: Example turbine power curve. 
In Region II, power varies cubically with wind speed in order to optimize energy capture. Both control strategies (speed and pitch) may be used in this region, although variable-speed operation typically makes pitch-control unnecessary once a constant, optimal pitch angle is set. The theoretical maximum power output for any turbine in this region is given by the Betz limit. Viscous losses and other non-ideal effects decrease the power output from this ideal value, but the goal of the control system is to approach this value as closely as possible.

Region III occurs above the rated wind speed. Rated power is maintained in this region to limit electrical and mechanical loads. Pitch-controlled turbines accomplish this by decreasing blade pitch. Typically, proportional-integral-derivative (PID) controllers are integrated with the pitch control mechanisms for this purpose. For stall-controlled turbines, outboard blade stalling limits power in Region III. Blade twist is commonly employed to provide a gradual stall over the span, but the complex aerodynamic nature of stall makes exactly maintaining rated power difficult.

The final region, Region IV, occurs above the cut-out wind speed. Operation in this region is completely halted and blade braking is employed in order to prevent damage to the turbine.

Power losses due to accumulated blade roughness occur on both pitch-controlled and stall-controlled turbines but are typically much larger on stall-controlled turbines. The primary reason is that pitch-controlled turbines (as well as active-stall turbines) can maintain rated power in Region III even in the presence of roughness, leading to only modest power losses of around 5\% [4]. Passive stall-controlled tur- 
bines, on the other hand, can exhibit power losses up to $25 \%$ in Region III because stall behavior is heavily influenced by roughness [5].

\section{I.B. Insect-Induced Performance Degradation}

A common cause of roughness on wind turbines is insect impingement upon the blade surface. At low flow velocities or large impingement angles, the insects may skim across the blade surface and leave little residue behind. However, for high flow velocities and impact paths perpendicular to the blade surface, the insect carcasses can remain on the surface and create excrescences, as shown in Figure I.2. These excrescences cause deleterious effects on blade section performance including loss of lift and increased drag. Unlike erosion or paint chip roughness, insect roughness degrades blade performance almost immediately once the blade enters field operation.
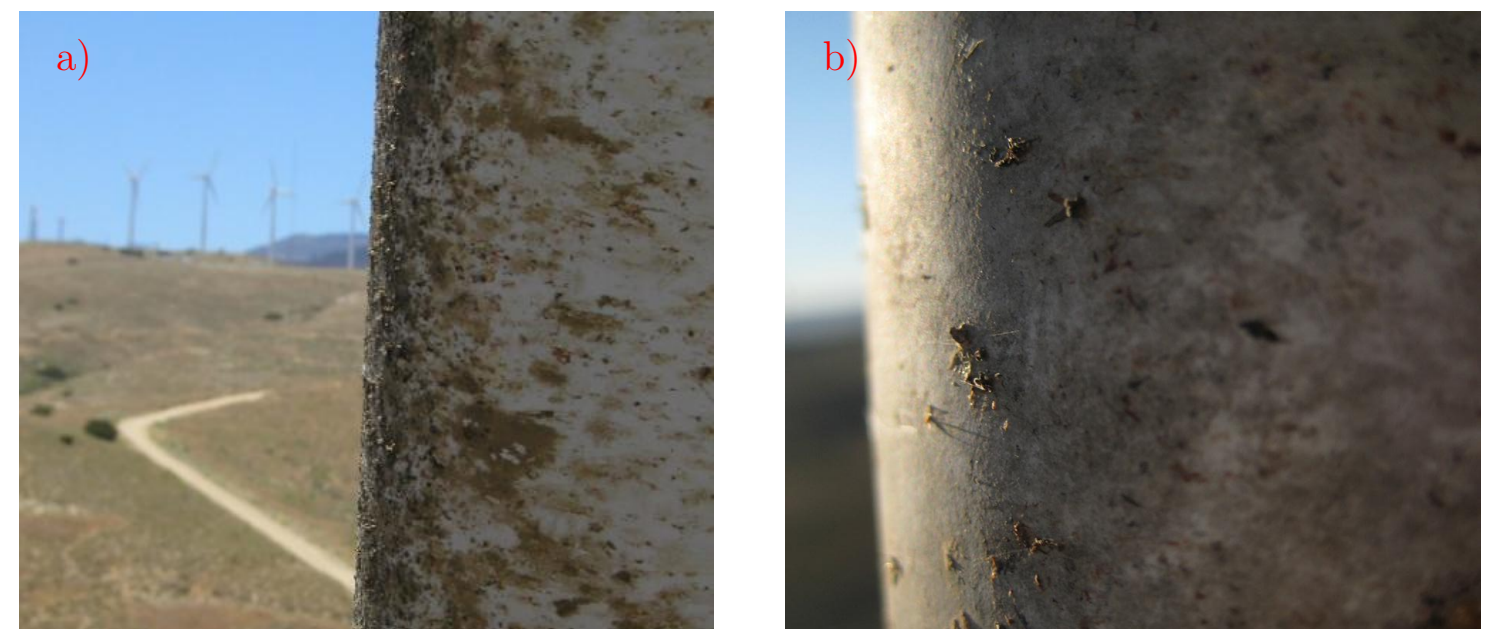

Figure I.2: Example insect roughness images. Images correspond to (a) heavy contamination [6] and (b) minimal contamination [7]. 
Most studies on insect-induced power degradation have been conducted on stallregulated turbines. Moroz and Eggleston observed power reductions of nearly $20 \%$ on a Bonus $120(120 \mathrm{~kW})$ wind turbine after just 15 days of field operation in San Gorgonio, California [8]. The Solar Energy Research Institute (now called the National Renewable Energy Laboratories, NREL) found similar results with 20-30\% losses in AEP on stall-regulated, $65 \mathrm{~kW}$ turbines [9]. A more recent study by Corten again revealed the insect roughness problem with a $25 \%$ loss in AEP for a $700 \mathrm{~kW}$ turbine [5].

Fewer studies exist showing performance losses on pitch-regulated turbines. Modern turbine manufacturers are hesitant to publish such data, and field measurements are difficult to obtain for research groups. Standish conducted wind tunnel tests of an $18 \%$ airfoil with clean, zig-zag trip tape, and a variety of grit roughness configurations [4]. He then used an aeroelastic solver called XBlade to predict the resulting AEP loss for each roughness configuration. His results revealed AEP losses of approximately $10 \%$ for a modern, pitch-regulated turbine, but he noted that this loss is likely more severe than what would be encountered in field operation. He suggested a more likely AEP loss of $5 \%$ as most blade roughness is less severe than his tested roughness.

Ehrmann provided the first set of field measurements showing roughness-induced power losses on a megawatt-scale, pitch-regulated turbine [7]. He analyzed four years of data and found approximately $4 \%$ power loss at speeds below rated wind speed. Interestingly, this power loss was only observed during dry months of the year, and no long-term decay was evident. This suggests that the losses he observed were 
due primarily to insect roughness which is washed away during the rainy months. Spruce's field measurements of a $1500 \mathrm{~kW}$, active-stall turbine reveal similar behavior [6]. He recorded power output over a four-month period and found that power losses were heavily correlated with rainfall. For severely contaminated blades caused by dry conditions, he measured power losses resulting in up to $13 \%$ loss in AEP.

Many studies have also been conducted showing the effect of insect roughness on airfoil performance. Boermans \& Selen attached sheets of self-adhesive matted polyester films on the wings of sailplanes and used these to collect insects in-flight [10]. The insect-covered sheets were tested on wind tunnel models of sailplane airfoils to give accurate measurements of actual insect contamination. An artificial bug pattern consisting of small squares of duct tape of $330 \mu \mathrm{m}$ thickness placed on the leadingedge was also tested but failed to reproduce the actual insect results except at high lift coefficients. At low angles of attack, the drag polar of the insect-covered airfoils converged to the clean airfoil polars. The height of Boermans \& Selen's insects was not measured, but Coleman shows that $330 \mu \mathrm{m}$ is a low estimate for the height of excrescences left by fruit flies or house flies [11].

Moroz \& Eggleston used similar adhesive strips to capture insects on $\mathrm{kW}$-scale turbines [8]. The insects from these strips were scanned using laser profilometry and a mold of the insect patterns was created. The maximum insect height they measured varied for different turbines between 750 and $1,370 \mu \mathrm{m}$, similar to the largest flies measured by Coleman [11]. Their insect moulds were tested on a NACA 4415 blade and compared to grit roughness results on the same blade. Their results, like those of Boermans \& Selen, show that actual insect roughness is well-represented by grit 
only at large angles of attack. At low angles of attack, the drag of the insect mold roughness was similar to that of the clean airfoil.

White et al. tested a NACA $63_{3}-418$ airfoil under clean, tripped, low- $k$ and high- $k$ roughness configurations ( $k$ here refers to roughness height) and at Reynold numbers of $1.6 \times 10^{6}$ and $3.0 \times 10^{6}$. Results were compared to "standard roughness" results given by Abbott \& von Doenhoff [12]. The airfoil was manufactured with a removable leading-edge which could be replaced with rapid-prototyped leading-edges representing each roughness configuration. The maximum roughness height for the low- $k$ and high- $k$ configurations was 70 and $1,200 \mu \mathrm{m}$, respectively. Results were similar to those of Boerman \& Selens and Moroz \& Eggleston for the low- $k$ roughness. For high- $k$ roughness, drag was nearly twice that of "standard roughness" and even exceeded that of the tripped configuration.

Soltani tested a $660 \mathrm{~kW}$ turbine airfoil using $500 \mu \mathrm{m}$ roughness [13]. His approach differed from that of previous researchers in that he applied roughness over the entire upper surface rather than just at the leading-edge. He observed a $35 \%$ decrease in maximum lift coefficient due to this roughness. Neither trip strip nor standard grit-roughness approaches were able to reproduce this result. This suggests that roughness distribution is a factor in determining performance behavior. Certainly, there is ample evidence that standard roughness testing techniques do not capture the full behavior of insect roughness. 


\section{I.C. Historical Airfoil Design}

Early wind turbine blades used airfoils which were originally designed for aircraft such as the NACA 44XX, NACA 23XX, NACA 63XXX, and NASA LS(1) series. These airfoils all suffered large performance losses from leading-edge contamination as well as problems with excessive power in high winds and low power-to-thrust ratios leading to wind farm array losses [14]. In 1984, NREL began work on a series of airfoils designed specifically for use on wind turbine blades. Their goals for these airfoils were better power regulation, decreased sensitivity to roughness, greater annual energy output, and higher power-to-thrust ratios [9]. Initial design work was done using the Eppler Airfoil Design code [15]. The code combines a panel method with a integral boundary layer solver in a viscous/inviscid iteration (VII) scheme. To simulate roughness, transition was forced at the leading-edge of the airfoils. Research conducted since this time has shown that forced transition models are unrepresentative of insect roughness [3] [16] [17]. Nevertheless, the NREL turbine blades showed vast improvements over the NACA and NASA blades in atmospheric tests. NREL blades were installed on turbines side-by-side conventional blades and showed 10-30\% improvement in AEP [18] [19] for dirty blades. This marked a substantial improvement over previous blade designs, but a $20 \%$ decrease in power due to roughness was still evident over the turbines' lifetimes [9].

Wind tunnel tests of the NREL blades have focused on using grit roughness to simulate field performance. The mid-series airfoils were tested at Ohio State University using a molded insect pattern taken from turbine field operation. The pattern was cut into a steel sheet with holes large enough for a single grit-roughness 
element. An element of standard \#40 lapidary grit was placed in each hole and transferred to the model using double-tack tape. The grit size used corresponds to a height-to-chord ratio (typically written as $k / c$, where $k$ is the roughness height and $c$ is the airfoil chord length) of 0.0019 . A $25 \%$ reduction in maximum lift and $60 \%$ increase in minimum drag was found for the NREL S814 airfoil at a Reynolds number of $1.2 \times 10^{6}[20]$. Tests of the NREL S815 model using the same techniques showed a $25 \%$ reduction in lift and $30 \%$ increase in drag [21].

Later tests of the thicker NREL blades were conducted by Somers in the Delft University of Technology (DUT) Low Speed Laboratory with the goal of verifying predictions made by the Eppler Airfoil Design code [22]. The use of the Delft facilities allowed larger Reynolds numbers (up to $3.0 \times 10^{6}$ ) to be reached with turbulence levels around $0.04 \%$. Somers tested multiple roughness types including severe grit roughness and fixed-transition configurations. The severe grit roughness height varied between 380 and $640 \mu \mathrm{m}$ in height and was applied on a $51 \mathrm{~mm}$ wide strip at $2 \%$ chord and $10 \%$ on the model's upper and lower surfaces, respectively. For the fixed-transition case, a strip of grit roughness was also used but was sized only large enough to trip the boundary layer to turbulence. Maximum lift was decreased by $8 \%$ for the transition-fixed case and $23 \%$ for the severe roughness case compared to the clean configuration. Minimum drag increased by $50 \%$ for the transition-fixed case and $60 \%$ for the severe roughness case. The clean and transition-fixed results were replicated using the Eppler Airfoil Design code, but attempts were not made to match the severe roughness case. 
Turbine size has increased tremendously in the past decade. Megawatt-scale turbines with rotor diameters of over $100 \mathrm{~m}$ are becoming increasingly common, especially for offshore applications. With these larger turbines comes the need for thicker blades which can handle the increasing structural loads. Airfoils of $30-40 \%$ thickness are now necessary for the root sections of blades where structural loads are largest. In the 1980 s and early 1990s turbines commonly used NACA 4-series and 6-series airfoils whose thickness was linearly increased to provide the required stiffness. Unforeseen effects such as premature transition and increased roughness sensitivity severely degraded the performance of these turbines. With this in mind, researchers at Delft University began designing new turbine blades which were wellsuited for megawatt-scale turbines [23]. Initial design of the airfoils was accomplished using RFOIL, a modified version of the VII panel method code XFOIL [24]. RFOIL includes turbine rotational effects which can be used to predict transition and stall on rotating blades and was verified using wind tunnel results [25].

Additional wind tunnel tests of these airfoils were conducted specifically to examine the roughness sensitivity of large-thickness airfoils [25]. A variety of mid-tolarge thickness airfoils were tested. However, both roughness type (grit or zigzag trip tape) and test facilities varied between tests, making direct comparisons between airfoils difficult. Even so, it was clear that for airfoils up to $30 \%$ thickness, the most roughness-insensitive airfoils had a thin upper surface but a thick, "S"-shaped lower surface. This design maintains low pressure-gradients on the upper surface while still providing sufficient aft-loading of the blades to reduce structural loads. The absence of large, adverse pressure gradients on the upper surface was found to 
be crucial in preventing premature roughness-induced separation. For 35-40\% thick airfoils, roughness insensitivity could not be achieved while maintaining structural requirements.

Further roughness testing was done on the DU 97-W-300 airfoil at the cryogenic tunnel of the DNW in Cologne, Germany. Roughness was simulated using two methods: Carborundum \#60 grit roughness (average grain size of $250 \mu \mathrm{m}$ ) applied over the top $40 \mathrm{~mm}$ of the upper and lower surfaces, and $400 \mu \mathrm{m}$ zigzag trip tape applied at $5 \%$ and $10 \%$ chord on the upper and lower surfaces, respectively. Testing was conducted at Reynolds numbers between $1 \times 10^{6}$ and $10 \times 10^{6}$. The loss in lift and increase in drag of the Carborundum roughness was found to be nearly double that of the trip tape. However, the applied testing methods introduced considerable scatter in the data which calls the validity of these results into question.

Blade designers have acknowledged roughness-sensitivity as an issue for decades. A great deal of test data exists, but inconsistent roughness simulation techniques make comparison between different tests difficult. Recent trends toward increased airfoil thicknesses and Reynolds numbers further reduce the usefulness of early airfoil tests. Though much work has been done in reducing roughness sensitivity, it is clear that there is still a need for improved design methods and roughness simulation techniques.

\section{I.D. Roughness Effects on Boundary-Layer Transition}

The effect of roughness on boundary layer development is not well-understood, in part due to the large number of parameters which affect boundary layers. Surface 
roughness, pressure gradients, freestream conditions (velocity, viscosity, and turbulence intensity), thermal gradients, and surface vibrations all affect boundary layer development and are difficult to control precisely. Modifying these parameters can lead to different boundary layer transition mechanisms which change how a flow moves from laminar to turbulent. The most commonly studied transition mechanism on $2 \mathrm{D}$ wing sections is the growth of Tollmien-Schlichting (TS) waves in the boundary layer. TS waves enter a boundary layer at regions of large streamwise surface variation, such as the leading-edge, through a process known as receptivity. Once inside the boundary layer, TS waves can grow and eventually cause the boundary layer to break down into turbulence. Roughness affects the transition path by introducing additional receptivity sites, modifying the behavior of already-present TS waves, and in some cases even bypassing the TS mechanism completely.

Bypass mechanisms were studied extensively by Morkovin [26] but are still not well-understood. Instead, correlations relating roughness and flow parameters have historically been used to determine whether or not a flow will transition through a bypass. Nondimensional quantities such as $k / \delta_{k}^{*}$ and $k / \theta_{k}$ are most common. These relate $k$, the roughness height, to the typical boundary layer length scales, $\delta^{*}$ (displacement thickness) and $\theta$ (momentum thickness). The subscript $k$ indicates that values should be evaluated at the location of the roughness. Dryden compiled transition data from a number of researchers and successfully collapsed data for zeropressure gradient flows by using $k / \delta_{k}^{*}$ as the correlation parameter [27]. However, this method was unable to collapse data for flows with pressure gradients. 
Smith and Clutter suggest that neither $k / \delta^{*}$ nor $k / \theta$ are appropriate parameters since their use suggests a critical roughness height of zero at the leading edge [28]. They prefer the use of a roughness Reynolds number, first proposed by Schiller [29]. The roughness Reynolds number is given as $R e_{k}=u_{k} k / \nu_{k}$, where $k$ is the roughness height, $u_{k}$ is the velocity of an equivalent undisturbed boundary layer at this height and location, and $\nu_{k}$ is the corresponding kinematic viscosity at this location. The critical $R e_{k}$ is the value for which bypass occurs. The effect of pressure gradient is at least partially captured in the definition of $R e_{k}$ through the $u_{k}$ term, so critical $R e_{k}$ is expected to be insensitive to pressure gradient [30].

Roughness is generally broken down into three categories: 2D roughness (steps or strips), isolated or arrayed 3D roughness, and distributed 3D roughness. Boundary layers with 2D roughness show gradual, forward movement of the transition front as $R e_{k}$ increases. This behavior begins when $R e_{k}$ is between 40 and 260 and continues until transition occurs at the roughness element [28]. Isolated 3D roughness such as insect roughness shows "more critical" behavior, with the transition front moving forward rapidly once a critical roughness Reynolds number is reached. Tani found that the critical roughness Reynolds number on isolated, cylindrical roughness is proportional to $(k / d)^{2 / 5}$, where $k$ is the roughness height and $d$ is the roughness diameter [30]. Typical proportionality constants for this relationship lie between 600 and 900 such that $R e_{k, \text { crit }}=A \cdot(k / d)^{2 / 5}$ where $A=600-900$. Transition relationships for distributed roughness are less well-defined due to difficulties in defining a "typical" surface. Experiments by Downs suggest that the mechanism for supercritical distributed roughness is similar to bypass observed for isolated roughness [31]. 
Downs was not able to fully analyze the transition mechanism for subcritical distributed roughness but suggests that it may be associated with TS wave growth.

\section{I.E. Insect Impingement Modeling}

Transition behavior and performance losses depend not only on the height of roughness but also on its location. The first research into quantifying insect impingement locations was done in a series of wind tunnel tests by Coleman in 1959 [11]. In the tests, Coleman discharged live fruit flies from a tube into a wind tunnel containing a NACA 6-series airfoil. A flexible copper strip was stretched over the airfoil surface and used to collect the insects. This strip was removed from the airfoil after testing, and the resulting impingement patterns were examined under a microscope. The excrescence heights recorded by Coleman were related to Schiller's critical $R e_{k}$ correlations and used to predict which insects would most likely cause bypass transition [29]. Coleman's results suggest that insect remains spread over the surface such that excrescence height is independent of the size of the initial insects over most of the surface. The only region where this is not true is very near the leading edge where whole insects remain. He also conjectured that only the roughness near the leading edge would likely be large enough to cause aerodynamic losses.

Coleman's research provides an accurate method of testing the effects of insect contamination; however, wide-scale wind tunnel testing with live insects is typically impractical. Flight testing has proven to be a simpler method of studying impingement patterns. Initial tests by Johnson found that flight conditions can partially erode collected insects and that flight through rainclouds can wash wing surfaces 
clean [32]. This mirrors the results of turbine performance improving after rainfall [7]. Boermans and Selen conducted flight research which includes accumulation data such as frequency of impact and chordwise impingement limits for different sailplanes [10]. They found that the majority of insects are collected during takeoff and landing while angle of attack and freestream velocity are varying. This makes correlating the impingement patterns with these flight parameters difficult. Furthermore, the flow conditions experienced on wings are typically much different than the flow conditions found on wind turbines, so these results are unlikely to be of much value in determining turbine impingement patterns. Unfortunately, very little insect impingement field data is currently available for turbines.

Maresh \& Bragg [33] examined the role that airfoil geometry and flow conditions play in determining impingement patterns using computational methods. Their code solves the equations of motion for insect particles in a 2D airfoil flowfield and uses this to determine expected impingement locations. To accomplish this, the insect properties are first cast into a nondimensional mass parameter, $K$, with relates the insect inertia to pressure forces in the flowfield. The mass parameter $K$ was first defined by Bragg for aircraft icing simulations and is used in more recent icing simulations such as NASA Glenn's LEWICE code [34] . LEWICE couples a two-dimensional panel method with the Lagrangean particle tracking techniques used by Maresh \& Bragg to determine the water droplet impingement patterns on airfoils [35]. The similar size and mass parameters of water droplets and insects make tools such as LEWICE well-suited for adapting into insect simulations. 


\section{I.F. Research Objectives}

Although a variety of studies have addressed these issues, there remains a lack of knowledge about interactions between insect distribution, boundary layer state, and performance losses on megawatt-scale, pitch-regulated turbines. Performance losses for pitch-regulated turbines are expected to be much less severe than on stallregulated turbines, but it is still unknown how increases in rotor size affects performance. Particularly, there is evidence that increased blade thickness leads to increased roughness sensitivity. Large-thickness blades are now commonly employed on inboard and mid-span blade sections in order to combat structural loads. Furthermore, most design work has focused on making airfoil performance insensitive to boundary layer tripping. Common tripping methods such as trip tape have been shown to be unrepresentative of insect roughness, and nearly all studies neglect the effect that insect distribution has on performance.

This work addresses these issues as follows. First, a code is written which will determine insect impingement patterns on two turbine airfoils of varying thicknesses. This code is used to determine realistic roughness locations for wind tunnel testing of the two airfoils. One of these airfoils, a NACA $63_{3}-418$, has already been tested in previous work by Ehrmann [7]. A wind tunnel model of the other airfoil, an NREL S814, is designed and tested under a variety of realistic roughness configurations at Reynolds numbers from $1.6 \times 10^{6}$ to $4.0 \times 10^{6}$. Lift, drag, and pitching moment coefficients are collected for each configuration and Reynolds number. Transition behavior is analyzed using infrared (IR) thermography. A blade-element-momentum (BEM) code is written and used to predict losses in AEP for both airfoils and each 
roughness configuration. AEP losses and transition behavior of the two airfoils are compared.

This research benefits turbine designers and future researchers in the following ways. First, the work will provide turbine manufacturers with better understanding of the role of airfoil geometry on performance losses. This will allow manufacturers to produce more realistic power curves which will lower the risk for wind farm financiers. Second, the transition and airfoil performance data from the tests will be made available to researchers worldwide. This data is necessary for validation of computational fluid dynamics (CFD) models and performance estimation codes. In particular, the test is conducted in close cooperation with researchers from UC Davis who are developing a roughness-correlation-based CFD model for predicting airfoil performance losses. These codes will lead to better airfoil designs for minimizing roughness losses. Finally, data from the test will be used to verify empirical transition models under a variety of Reynold number, pressure gradient, and roughness conditions. 


\section{CHAPTER II}

\section{INSECT IMPINGEMENT ANALYSIS*}

An airfoil's susceptibility to insect-induced losses is governed by two factors: the stability of the boundary layer in the presence of roughness and the propensity of the airfoil to collect insects. The shape and operating conditions of the airfoil are important in determining the locations which insects are likely to collect. This section describes the simulation code, termed LEWBUG due to its similarity with NASA's LEWICE ice-accretion code, which is used to calculate insect impingement locations. LEWBUG was used to generate expected insect collection locations on several airfoils. Insect collection results guided the airfoil selection process and were used to modify the locations of roughness on the wind tunnel models to account for insect collection differences due to airfoil geometry.

\section{II.A. Trajectory Modeling}

The LEWBUG code is comprised of two main modules: a 2D, inviscid HessSmith panel method which is used to solve for the flow field around the airfoils and a Lagrangean particle tracking module which calculates the trajectories of insects inside this flow field. The panel method module code was programmed in $\mathrm{C}++$ and follows a procedure first used by Smith \& Hess [36]. First, the airfoil is discretized into $N$ panels $(N=400$ in this case) with half of the panels on the leading edge of the airfoil from $x / c=0.0$ to $x / c=0.3$ and half of the panels on the remainder

*Part of this chapter is reprinted with permission from "Computational analysis of insect impingement patterns on wind turbine blades" by B. Wilcox \& E. White, 2015. Wind Energy, Vol. 19, pp. 483-495, Copyright 2015 by John Wiley \& Sons, Ltd. 
of the airfoil. Each panel contains its own unknown source distribution as well as an unknown vorticity distribution which is constant over all panels. The potential functions of these panels are added to the potential function of the freestream flow to determine the full potential function of the flow. Determining the values for the source and vortex strengths requires the solution of $N+1$ equations, with $N$ of these equations determined by satisfaction of the no-penetration boundary condition at each panel's midpoint. The final equation comes from satisfying the Kutta condition at the airfoil's trailing edge. These equations are assembled into a $N \times N$ matrix and solved for the source and vorticity strengths. Lift coefficient is determined from the calculated vorticity strength using the Kutta-Joukowski theorem. Velocity can also be determined at any point in the flowfield by integrating the full potential flow function at that point.

The insect trajectories are computed next by using Lagrangean particle tracking in the body-fixed airfoil frame. The insects are assumed to be initially stationary with respect to the freestream velocity. Thus, the insect's initial velocity in the body-fixed frame can be written as the vector sum of the wind velocity and blade's rotational velocity. This initial velocity is prescribed to the insects at a location three chord lengths upstream of the airfoil's leading edge. As the insects near the blade, local changes in wind velocity induce drag forces which alter the insect's trajectory. The effect of these forces is determined by the insects' equations of motion, written in non-dimensional form using the insects' mass parameter $K$ : 


$$
\begin{aligned}
& \left.\ddot{\bar{x}}=-C_{D} / K \cdot\left((\dot{\bar{x}}-\cos \alpha)^{2}+(\dot{\bar{y}}-\sin \alpha)^{2}\right)\right) \cos \gamma \\
& \left.\ddot{\bar{y}}=-C_{D} / K \cdot\left((\dot{\bar{x}}-\cos \alpha)^{2}+(\dot{\bar{y}}-\sin \alpha)^{2}\right)\right) \sin \gamma
\end{aligned}
$$

where

$$
K=2 m /\left(\rho S_{r e f} c\right) .
$$

In these equations, $C_{D}$ is the insect drag coefficient, and $\bar{x}$ and $\bar{y}$ are defined parallel and perpendicular to the airfoil chord line, respectively. The airfoil angle of attack is given by $\alpha$ and the angle between the insect's velocity and the relative wind velocity is given by $\gamma$. The mass parameter $K$ is defined using the insect mass $m$, the density of air $\rho$, a particle reference area $S_{\text {ref }}$ (the same reference area used to calculate drag coefficient), and the airfoil chord length $c$. Insects with low $K$ values follow streamlines closely, and $K \rightarrow 0$ represents a limiting case where insects follow streamlines exactly. Insects with high $K$ values are less affected by changes in the surrounding flow field and tend to follow relatively straight, ballistic paths. Figure II.1 demonstrates particle trajectories for various values of $K$.

The insects in this study are modeled as particles, so their orientation cannot be known. We also assume that the insects do not consciously react to changes in relative wind velocity near the blade. These assumptions allow lift forces to be neglected in Equations 2.1 and 2.2. An average drag is also used which is independent of the insect's orientation. Because orientation is not considered, the insects are essentially represented as spherical.

For a large range of Reynolds numbers (approximately 4,000 to 200,000), the drag coefficient of a sphere is approximately constant. It is therefore appropriate to 

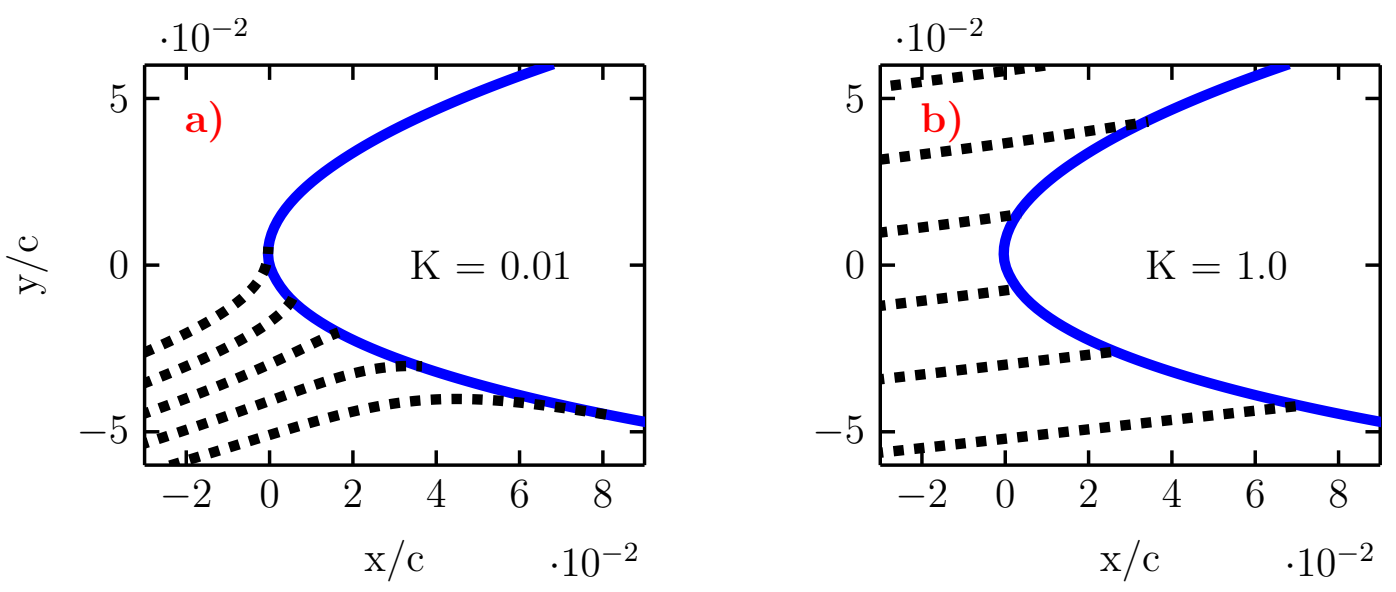

Figure II.1: Example insect trajectory plots. Plots correspond to (a) low- $K$ and (b) high- $K$ particles.

assume that the drag coefficient of an insect will be constant over a similar range. Reynolds numbers are computed using the insect length scale and difference in the insect's velocity and wind velocity, both of which are small values. This makes it unlikely that Reynolds number will exceed the upper limit of the constant drag model, so constant drag is used in the entire high Reynolds number regime. In the low Reynolds number regime, the constant-drag assumption no longer applies. Instead, a modified Stokes' Law is used in which drag coefficient varies with the inverse of Reynolds number. A piecewise model is used to transition between the two models in the different flow regimes:

$$
C_{D}= \begin{cases}C_{D, r e f} R e_{r e f} / R e & : R e<R e_{r e f}=4000 \\ C_{D, r e f} & : R e \geq R e_{r e f}=4000\end{cases}
$$

Flow field data and discretized forms of Equations 2.1 and 2.2 are used with this drag model to compute the insect trajectories from initial conditions. 


\section{II.B. Insect Population Modeling}

Solving the insect equations of motion requires knowledge of the insects' masses, references areas, and drag coefficients. Experimentally determining these parameters was beyond the scope of this work. However, many resources exist which contain this data. Researchers have used many methods of calculating insect drag including direct force measurements, correlations between maximum metabolic power and flight speed, and theoretical calculations [37]. Much of this work has been compiled by Vogel [38].

In order to accurately simulate expected impingement patterns on wind turbines, insects which are common to wind farms should be selected. The selected species should also be representative of the insect population as a whole. Common species of insects vary heavily between wind farms with geographical location. However, research has shown that individual shape differences between different species in the same insect group only marginally affects the insects' impingement patterns [11]. Differences between species can therefore be characterized almost entirely by mass parameter $K$. The insect order Diptera contains the majority of the small insects which contribute to blade contamination. The housefly, a species for which experimental data is readily available, is contained within this order and was determined to well represent the total insect population. Most importantly, this fly was used by Coleman in his experiments [11] which makes it ideal for validation of the LEWBUG code. To take advantage of this, the housefly was chosen to calculate impingement patterns in this study. 


\section{II.C. Blade Modeling}

The NREL 5 MW offshore reference turbine is commonly used to test new blade designs [39]. The turbine is variable-speed, pitch-controlled, and is rated for $5 \mathrm{MW}$ at $11.4 \mathrm{~m} / \mathrm{s}$. It has a $90 \mathrm{~m}$ hub height, $126 \mathrm{~m}$ rotor diameter, and cut-in and cut-out velocities of $3 \mathrm{~m} / \mathrm{s}$ and $25 \mathrm{~m} / \mathrm{s}$, respectively. The blade is linearly tapered from approximately $4.5 \mathrm{~m}$ near the hub to $1.5 \mathrm{~m}$ at the tip. The greatest power output for the NREL 5 MW reference turbine occurs for a tip-speed-ratio (ratio of tip rotational speed to wind speed) of 7.55. This turbine is used throughout this study for determining insect impingement patterns and AEP losses.

Comparisons of insect impingement patterns on different airfoils can be made at constant angle of attack, constant lift coefficient, or even constant lift-to-drag ratio. Of these parameters, lift coefficient generally has the greatest effect on total power output. For this reason, airfoils in this study are compared at constant lift coefficients so that power is approximately constant between comparisons. Ehrmann originally simulated his airfoil at an angle of attack of $6^{\circ}$, resulting in a lift coefficient of 1.1. This operating condition is near the airfoil's maximum lift and is likely too high for modern, variable speed turbines. However, it is used here to facilitate direct comparisons between airfoils.

\section{II.D. Insect Collection Parameters}

Originally used in aircraft icing experiments, collection efficiency is a measure of the likelihood and locations of particle impingement on an airfoil surface. The collection efficiency relates the mass flux of particles in the freestream to the mass flux 
which impinges upon the airfoil surface through Equation 2.5. In these simulations, collection efficiency is a measure of insect mass collection per unit time and unit blade surface area.

$$
\phi_{m}=\beta(A P C) U .
$$

Here, $\phi_{m}$ is the mass flux impinging upon the airfoil surface for a unit span, $\beta$ is the collection efficiency, $A P C$ is the "atmospheric particle content," and $U$ is the effective wind velocity combining freestream and blade rotational velocities. "Atmospheric particle content" is defined as the particle mass per unit volume in the freestream and represents the density of insects in the atmosphere. The collection efficiency also describes the movement of insect trajectories relative to one another. Two trajectories can be thought to form a tube of particles between them. As the distance between the trajectories widens, the tube widens and insects are spread over a larger distance. The collection efficiency describes the widening of this particle tube in differential form through $\beta=d y_{0} / d s$, where $d y_{0}$ is the distance perpendicular to effective wind velocity between two particles far upstream and $d s$ is their separation on the airfoil surface. Figure II.2 illustrates this distance.

The wrap distance $s$ is defined as a curvilinear distance along the airfoil surface. The wrap distance is defined starting at the airfoil's leading edge and increasing toward the upper surface. Upper surface coordinates are given by positive values of $s$ and lower surface coordinates are given by negative values. Figure II.3 shows these coordinates.

An additional parameter which is used to classify mass impingement is the insects' impact velocity, the insect velocity normal to the blade surface at the point 


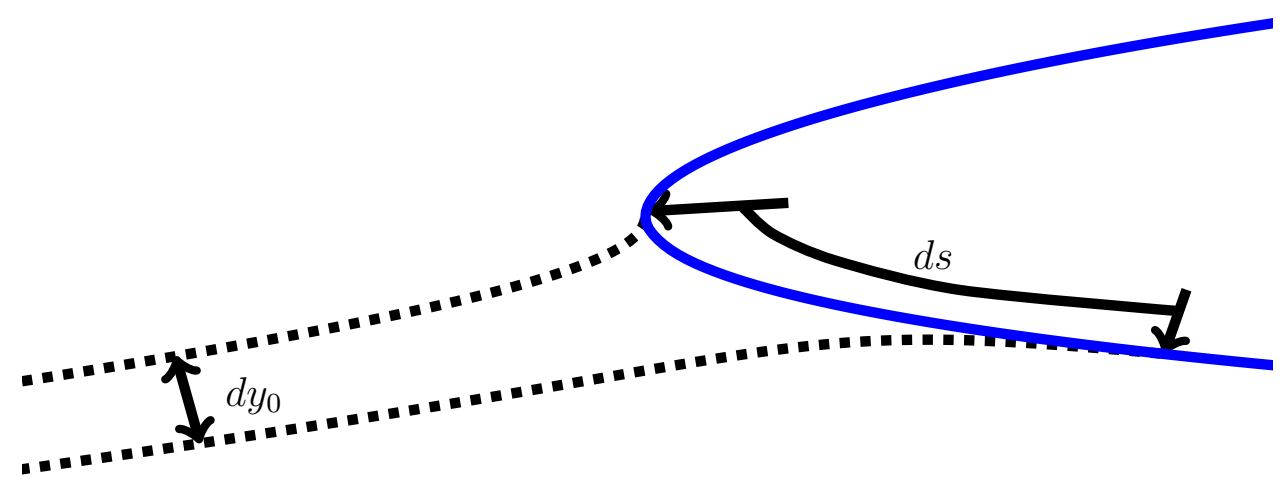

Figure II.2: Collection efficiency definition diagram.

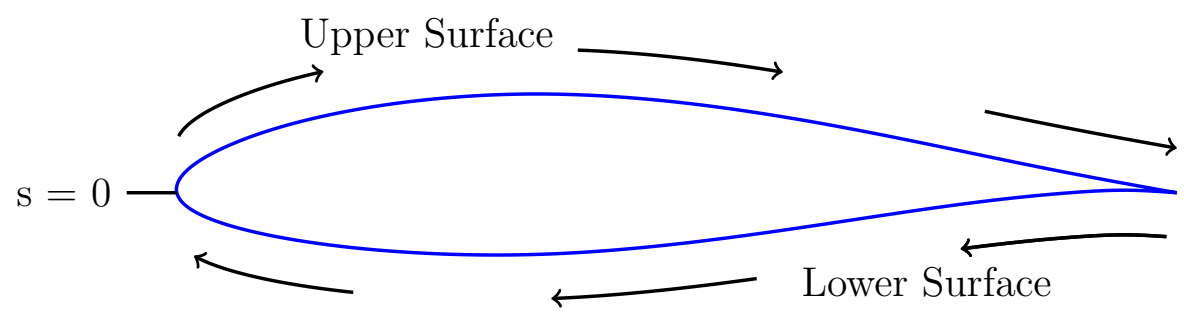

Figure II.3: Wrap distance definition diagram.

of contact. Coleman shows that this velocity is directly related to the formation of excrescences on the airfoil surface [11]. He defines a "rupture velocity" as the minimum impact velocity for which an excrescence will be created. Insects with impact velocities larger than their rupture velocity are likely to adhere to the surface. Furthermore, larger impingement angles typically correspond to larger excrescence heights since insects which hit a surface more directly are less likely to smear over the surface. Krishnan observed that insects which impact the surface at angles near $90^{\circ}$ typically leave in-tact exoskeletons on the surface whereas those that impact at lower angles leave behind only a hemolymph residue [40]. Impact velocity is a dimensional quantity, however, which can obscure comparisons between different 
operating conditions. To combat this, both impact velocity and collection efficiency are utilized here.

\section{II.E. Airfoil Selection}

Preliminary testing of the NACA $63_{3}-418$ was underway during the development of LEWBUG. Once completed, the first task of LEWBUG was to to guide selection of a thicker airfoil to act as a counterpart to the relatively thin NACA $63_{3}-418$. Comparing insect distribution patterns was crucial for selecting this airfoil. A variety of other requirements were also considered in the selection process:

1. The airfoil must be notably thicker than the $18 \%$ thick NACA $63_{3}-418$ used by Ehrmann [7].

2. The airfoil must exhibit markedly different insect collection patterns than the NACA $63_{3}-418$.

3. The airfoil must be relevant to modern wind turbines.

4. The airfoil profile must be available in open literature.

5. Performance data for the airfoil should be available in open literature for test verification.

6. The airfoil shape should be useful for validation of CFD software.

Based on these requirements, the following group of airfoils was chosen for consideration: the NACA $63_{4}-421$ (21\% thick), NREL S814 (24\% thick), DU 91-W2-250 (25\% thick), and DU 97-W-300 (30\% thick). Wind tunnel data is available for all of 
these airfoils [12] [22] [41] [17] [23], but data for the DU airfoils may be more difficult to access.

To compare differences in insect collection patterns, each airfoil's collection efficiency was computed using LEWBUG at a location corresponding to $80 \%$ span on the NREL 5 MW reference turbine. Insects are most likely to fly during times when wind speed is low but are most likely to rupture at high speeds, so a moderate wind speed of $8 \mathrm{~m} / \mathrm{s}$ was used.

Figure II.4 compares the collection efficiency of the possible airfoil choices with that of the NACA $63_{3}-418$ for a lift coefficient of 0.6 . The collection efficiency diagrams reveals that the thicker airfoils have a larger extent of high collection efficiency on the lower surface, but upper surface collection efficiency is similar between all airfoils. This is because turbine airfoils typically have increased thickness on the lower surface but only marginally thicker upper surfaces. The NACA $63_{4}-421$ is the only airfoil whose collection efficiency diagram is not markedly different from those of the NACA $63_{3}-418$. This is likely due to the small thickness variation between the two airfoils.

Pressure distributions of the airfoils for a lift coefficient of 0.6 were compared using XFOIL [24]. The pressure coefficient, $c_{p}$, plots are shown in Figure II.5. The NREL S814 and DU 97-W-300 plots both show long regions of nearly constant pressure gradient on the lower surface. These regions provide an ideal location for researchers at UC Davis to validate their CFD model before moving on to the highly varying pressure distributions on the upper surface. However, the very large pressure 


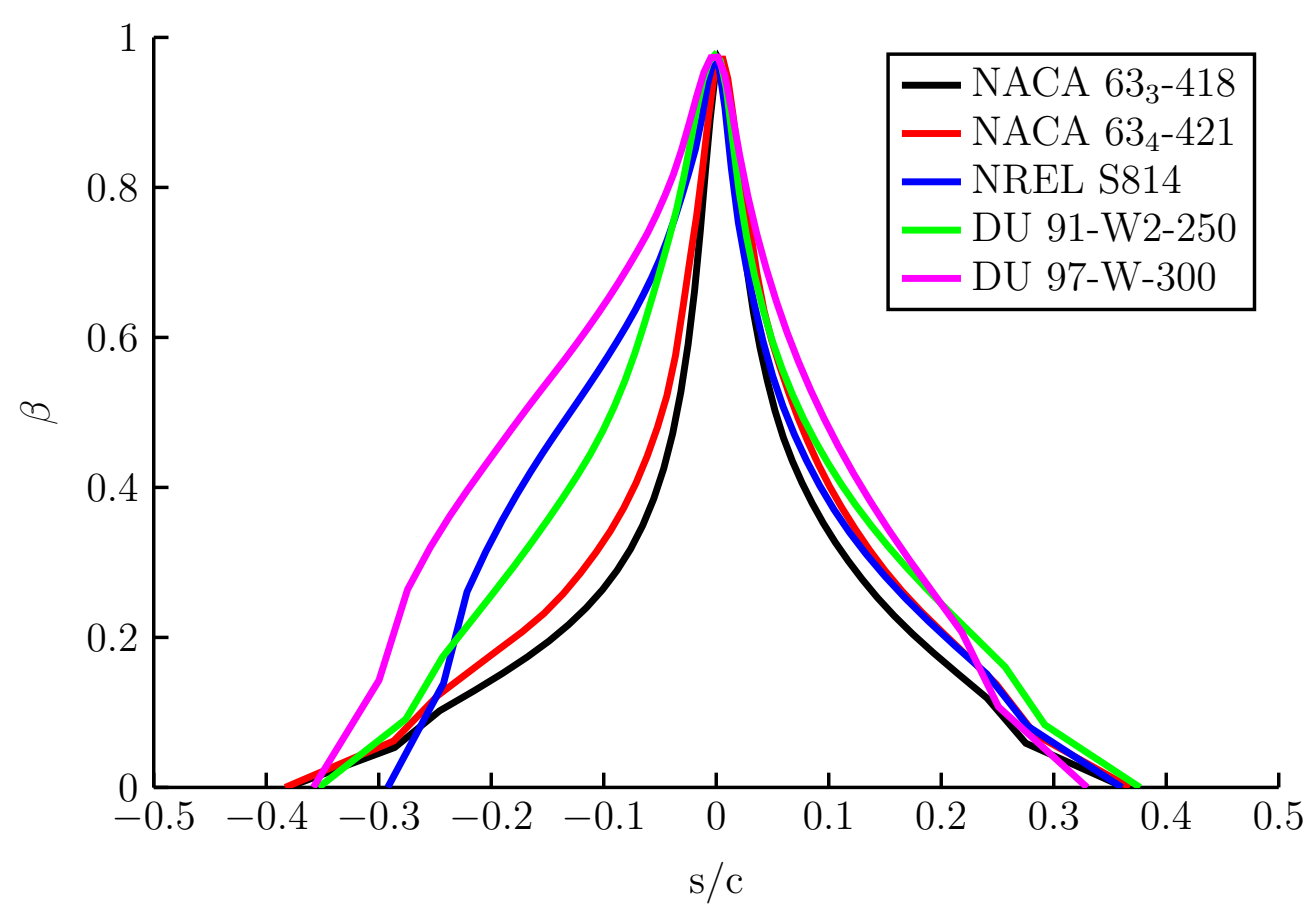

Figure II.4: Airfoil collection efficiency comparisons.

gradients present on the DU 97-W-300 are outside typical validation ranges for the model.

Based on the availability of previous test data, benefits for code validation, and large differences in collection efficiency from the NACA $63_{3}-418$, the NREL S814 was chosen as the airfoil for wind tunnel testing. Figure II.6 shows the profiles of the NACA $63_{3}-418$ and NREL S814 airfoils. The upper surfaces of the two airfoils are similarly shaped, but the lower surface of the NREL S814 has added thickness and a distinctive 'S' shape which is not present on the NACA $63_{3}-418$. The NREL S814 airfoil coordinates are located in Appendix A. 

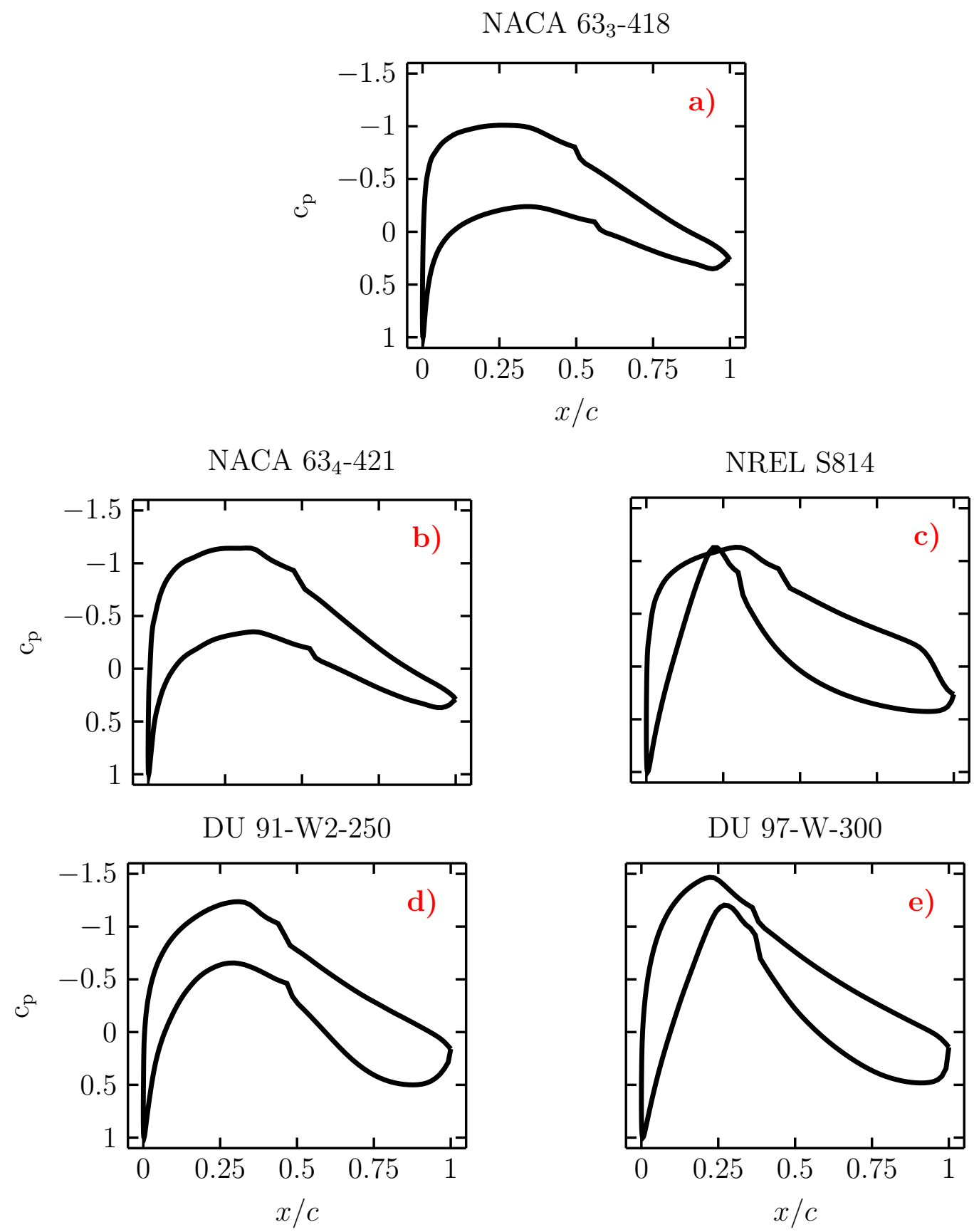

Figure II.5: Airfoil pressure coefficient comparisons. 

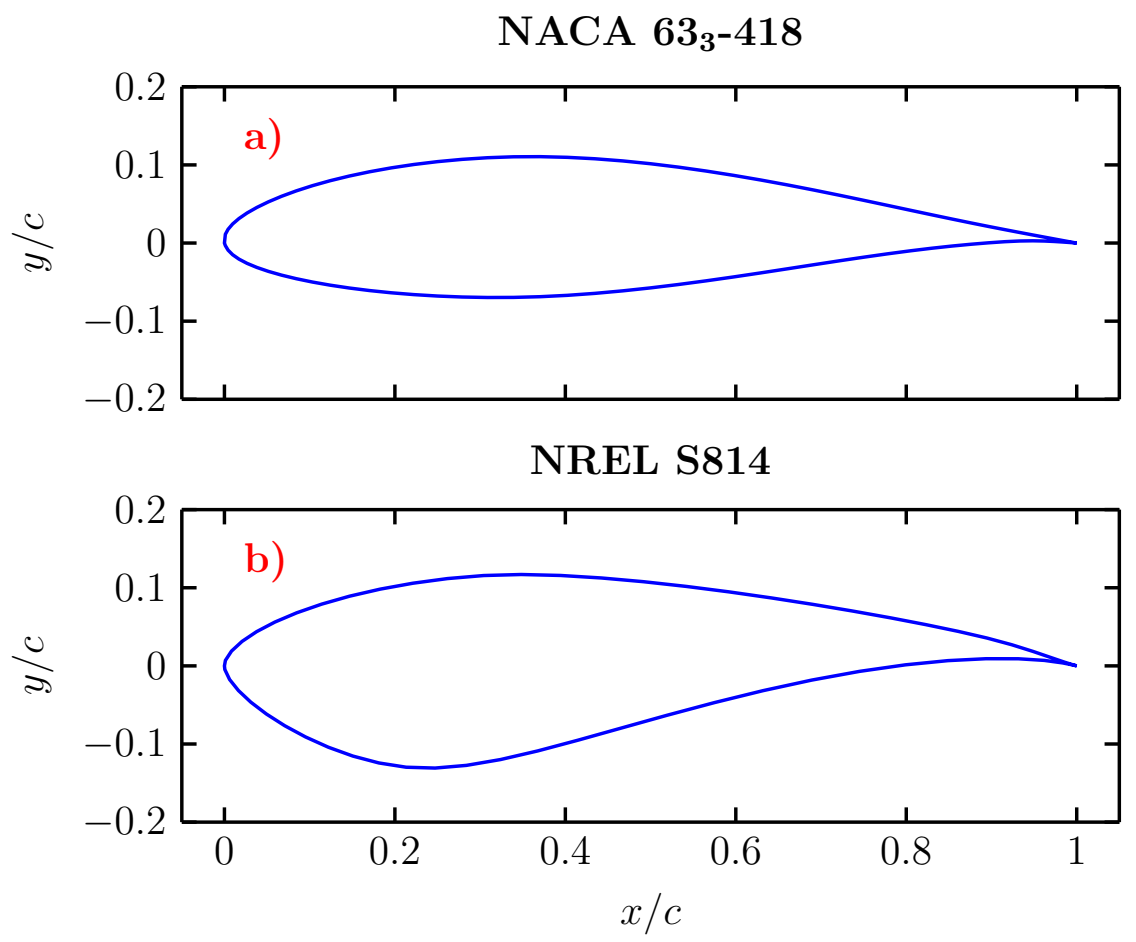

Figure II.6: Airfoil profiles. Profiles correspond to (a) NACA $63_{3}-418$ and (b) NREL S814 airfoils.

\section{II.F. Insect Distribution Results}

Collection efficiency diagrams of the NACA $63_{3}-418$ and NREL S814 for the operating conditions discussed in Section II.E are shown in Figure II.7. On the upper surface, the collection distribution of the two airfoils is very similar due to their similarity in upper-surface shape. On the lower surface, however, the NREL S814 shows increased collection efficiency over most of the surface but has a decreased maximum roughness extent. The decrease in maximum roughness extent is explained by the distinctive "S" shape of the NREL S814's lower surface. The airfoil's large thickness near $25 \%$ chord shields the remaining $75 \%$ of the airfoil from insect impingement. 
Consequently, there is unlikely to be much insect roughness on the downstream portion of this airfoil.

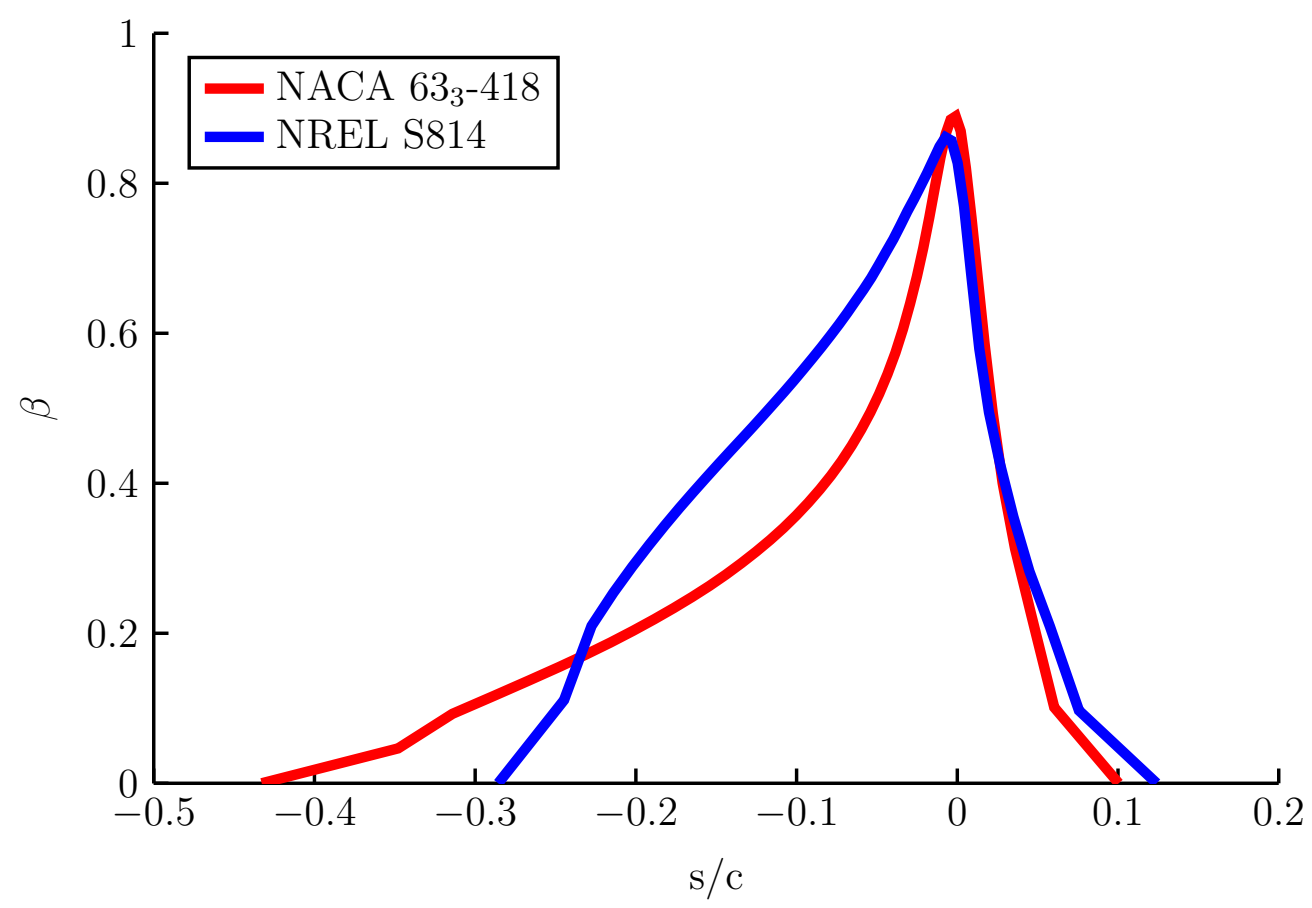

Figure II.7: NACA $63_{3}-418$ and NREL S814 collection efficiency diagrams.

Despite the region of nonzero collection efficiency being larger, the NACA $63_{3^{-}}$ 418 likely collects fewer insects than the NREL S814. The reason for this is that insects will not adhere to a surface unless their impact velocity exceeds the rupture velocity. Figure II. 8 shows the distribution of impact velocities overlayed on the collection efficiency diagram for the NACA $63_{3}$-418. Impact velocities are shown with dots and nondimensionalized by the equivalent wind velocity which combines the blade and wind velocities. For ballistic particles, these nondimensional impact velocities are completely defined by the impingement angles. The impingement angles also 
directly relate to collection efficiency because the distance between two neighboring particles is dependent on the angle of their trajectories near the surface. As shown in Figure II.8, this causes the shapes of the collection efficiency and nondimensional impact velocitiy plots to be nearly identical. Accordingly, the impact velocity and collection efficiency can be used interchangeably for large- $K$ particles such as the insects studied here.

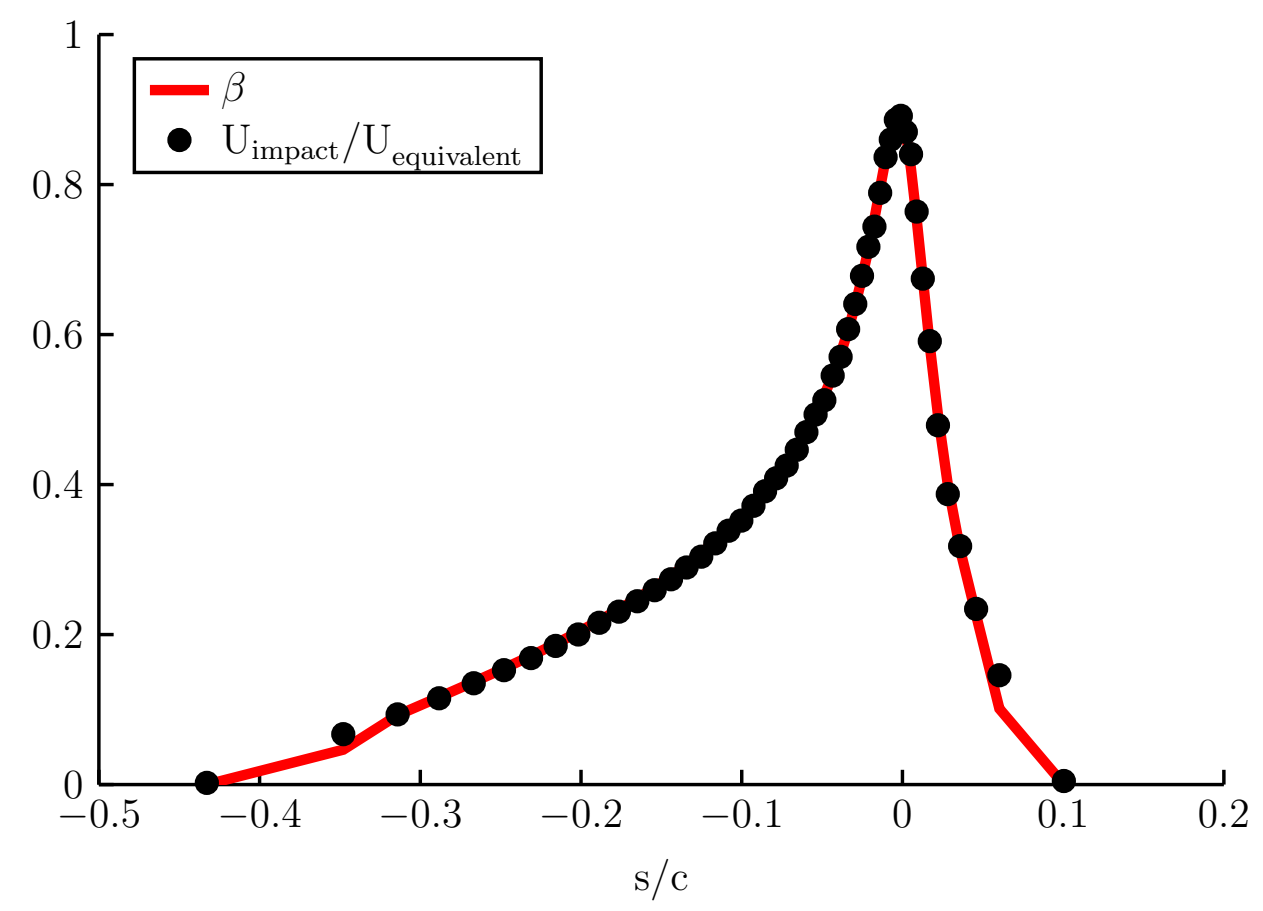

Figure II.8: Collection efficiency and impact velocity comparison.

Full turbine simulations were conducted by discretizing the NREL $5 \mathrm{MW}$ turbine and assuming that the blade was composed entirely of NACA $63_{3}-418$ and NREL S814 airfoils operating at lift coefficients of 1.1. Impact velocity distributions were computed at different spanwise locations and combined to create 3D distribu- 
tion diagrams. These are shown in Figures II.9 and II.10. Larger impact velocities are observed at outboard sections of the blade due to the blade's large velocity in these areas. Coleman gives the rupture velocity of a housefly as $12 \mathrm{~m} / \mathrm{s}$ [11]. This is shown in Figures II.9 and II.10 as thick black contour lines. The rupture velocity is not exceeded for sections inboard of approximately $15 \mathrm{~m}$, so no contamination is expected to occur here. As distance from the blade hub is increased, the impact velocity is also increased such that rupture velocity is exceeded over a larger percentage of the chord. The rupture velocity is exceeded over a larger portion of the NREL S814 airfoil, so this airfoil should show increased insect collection over the NACA $63_{3}-418$.

Blade chord length also decreases for outboard sections, resulting in larger values of mass parameter $K$ and higher collection efficiencies at outboard sections [42]. Figures II.11 and II.12 show spanwise variation in collection efficiencies for the two airfoils. A $30 \%$ collection efficiency contour is given by the thick black line. The chordwise extent of high collection increases as distances from the hub increases. This variation is much less than the spanwise variation in impact velocities, suggesting that most of the changes in impact velocities come from local changes in blade velocity rather than in changes in collection efficiency. 


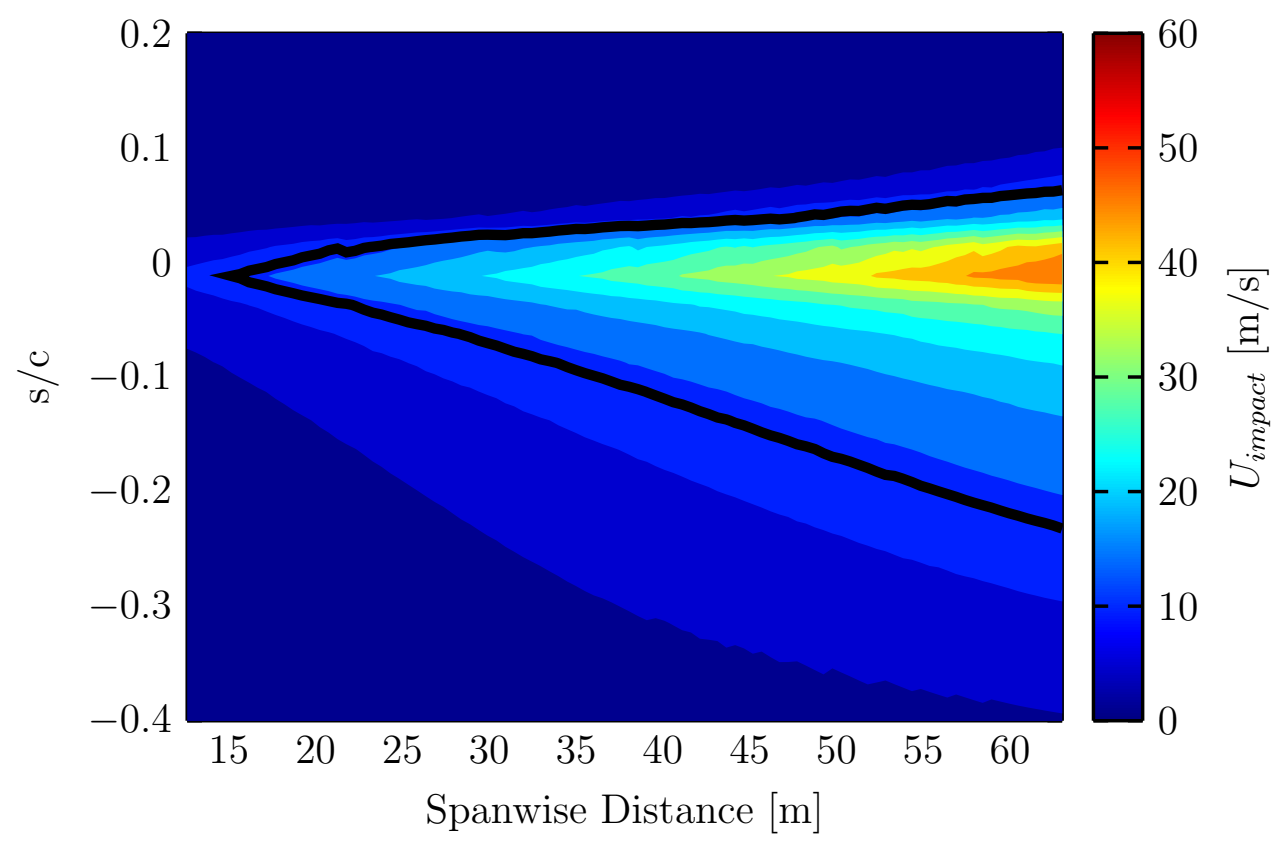

Figure II.9: Impact velocity distribution for the NACA $63_{3}-418$ turbine.

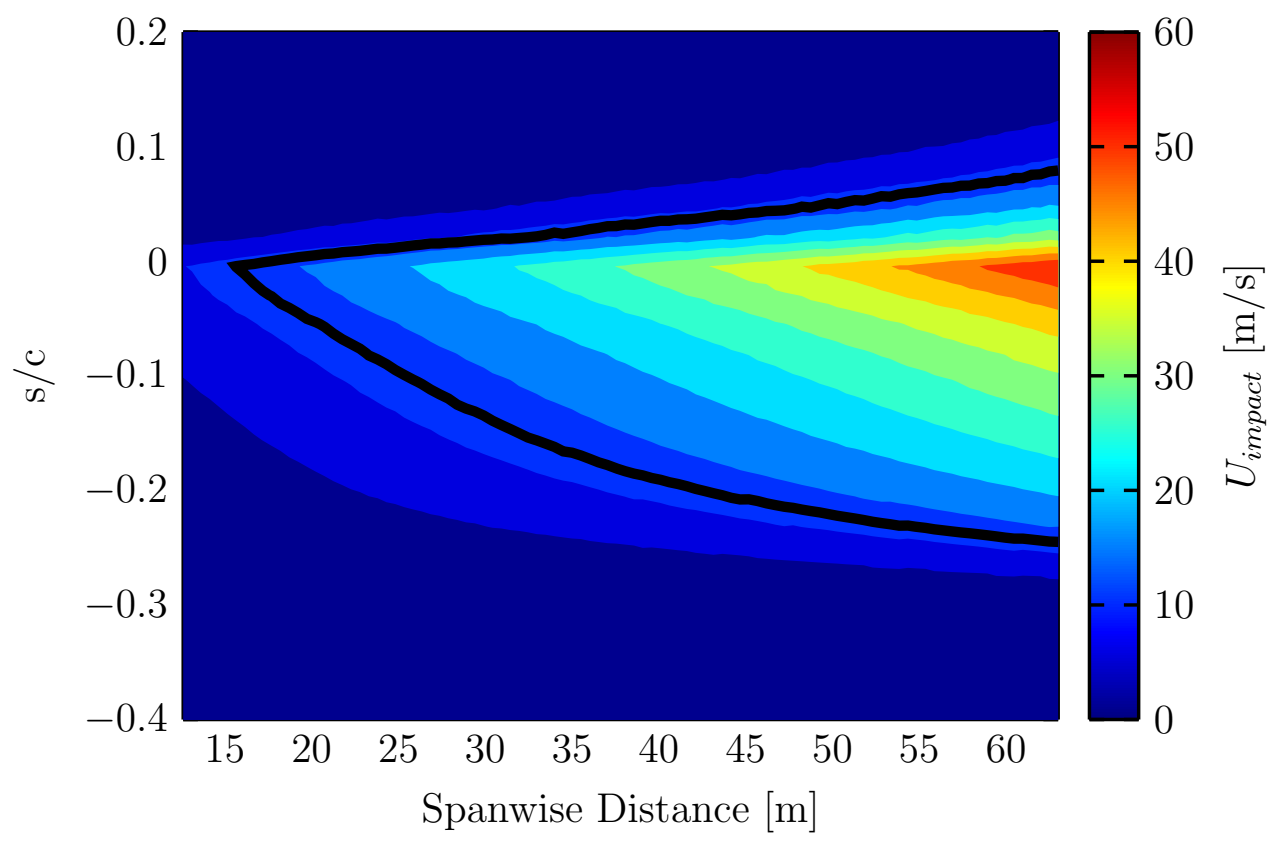

Figure II.10: Impact velocity distribution for the NREL S814 turbine. 


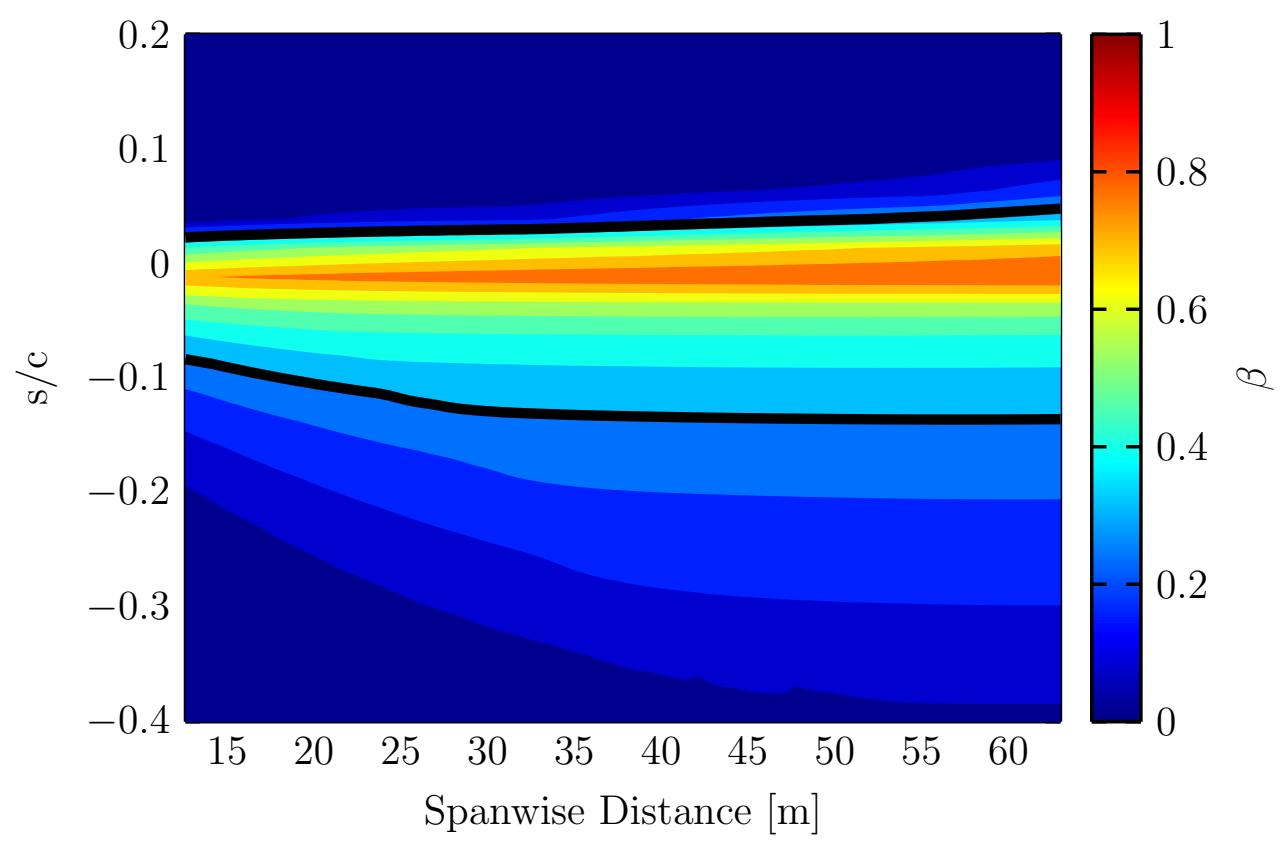

Figure II.11: Collection efficiency distribution for the NACA $63_{3}-418$ turbine.

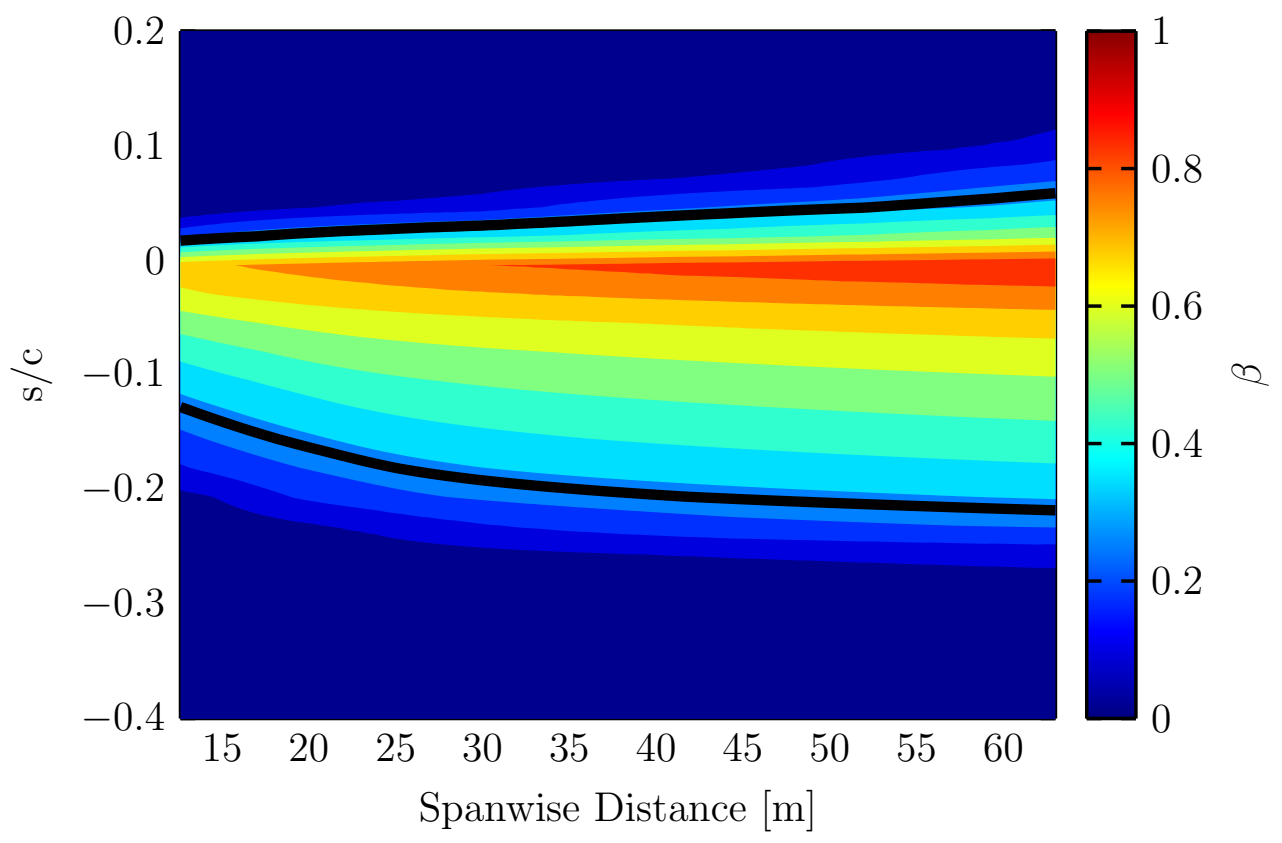

Figure II.12: Collection efficiency distribution for the NREL S814 turbine. 


\section{CHAPTER III}

\section{WIND TUNNEL TESTING}

Aerodynamic performance losses due to roughness are characterized most easily through wind tunnel testing. This section describes the experimental methods used during wind tunnel tests of the NREL S814 model. Experimental facilities, model design, roughness simulation methods, test instrumentation, and data analysis techniques are discussed, and results for airfoil lift, drag, pitching moment, and transition location are presented for different roughness configurations and Reynolds numbers. Wind tunnel testing of the NACA $63_{3}-418$ model was completed by Ehrmann [7] prior to this research, so testing methods for that airfoil are not discussed (although they are similar to the methods described here). However, results from both airfoils are presented and compared. The role of airfoil thickness on roughness sensitivity is a key motivation of this work, so comparisons between the $18 \%$ thick NACA $63_{3}-418$ and the $24 \%$ thick NREL S814 are the most important results presented here.

\section{III.A. Wind Tunnel Facilities}

Testing occurred in the Oran W. Nicks Low-Speed Wind Tunnel (LSWT) at Texas A\&M University. The LSWT is a closed-loop tunnel with a $7 \mathrm{ft}$ by $10 \mathrm{ft}$ test section and can achieve freestream velocities up to $90 \mathrm{~m} / \mathrm{s}$. Each corner of the test section has $1 \mathrm{ft}$ chamfers, reducing the total test section area to $68 \mathrm{ft}^{2}$. The floor and ceiling of the test section diverge 1 inch over its $12 \mathrm{ft}$ length in order to correct for boundary-layer growth. Vents at the end of the test section ensure that the static pressure inside the tunnel is equal to the ambient pressure inside the LSWT control 
room. Test section velocity is calculated by measuring the static pressure difference between pressure taps in the settling chamber and the test section inlet. The relationship between this pressure difference and the test section dynamic pressure was determined by calibration against a Pitot-static probe placed in the center of the empty test section. Tunnel temperature is measured with a thermocouple mounted at the test section entrance. A six-component balance is located beneath the test section. Balance data was not used for this test, but the balance still provides a convenient model mounting interface. The balance mount includes a turntable assembly which allows models to be rotated up to 350 degrees. Barometric pressure is measured underneath the tunnel in the balance room. A humidity sensor is mounted at the test section outlet to account for variations in air density. Temperature, velocity, and static pressure measurements are used to hold Reynolds number within $1 \%$ of its target value throughout the test. A two-axis traversing mechanism is mounted at the test section outlet. The traverse is capable of 60 inches of lateral movement and 40 inches of vertical movement, centered at the test section center. Figure III.1 shows a cutaway view of the wind tunnel test section with a vertically-mounted blade model. The turntable assembly, traversing mechanism, wake rake, compliant bearing, and test model are all visible. These are discussed in more detail in Ehrmann et al. [7] and Section III.B.

Tunnel flow quality tests were conducted using hotwire anemometry. Hotwires measure flow velocity based on the balance of the forced-convection heat transfer to the flow and power dissipated inside the wire. The convective heat transfer given by 


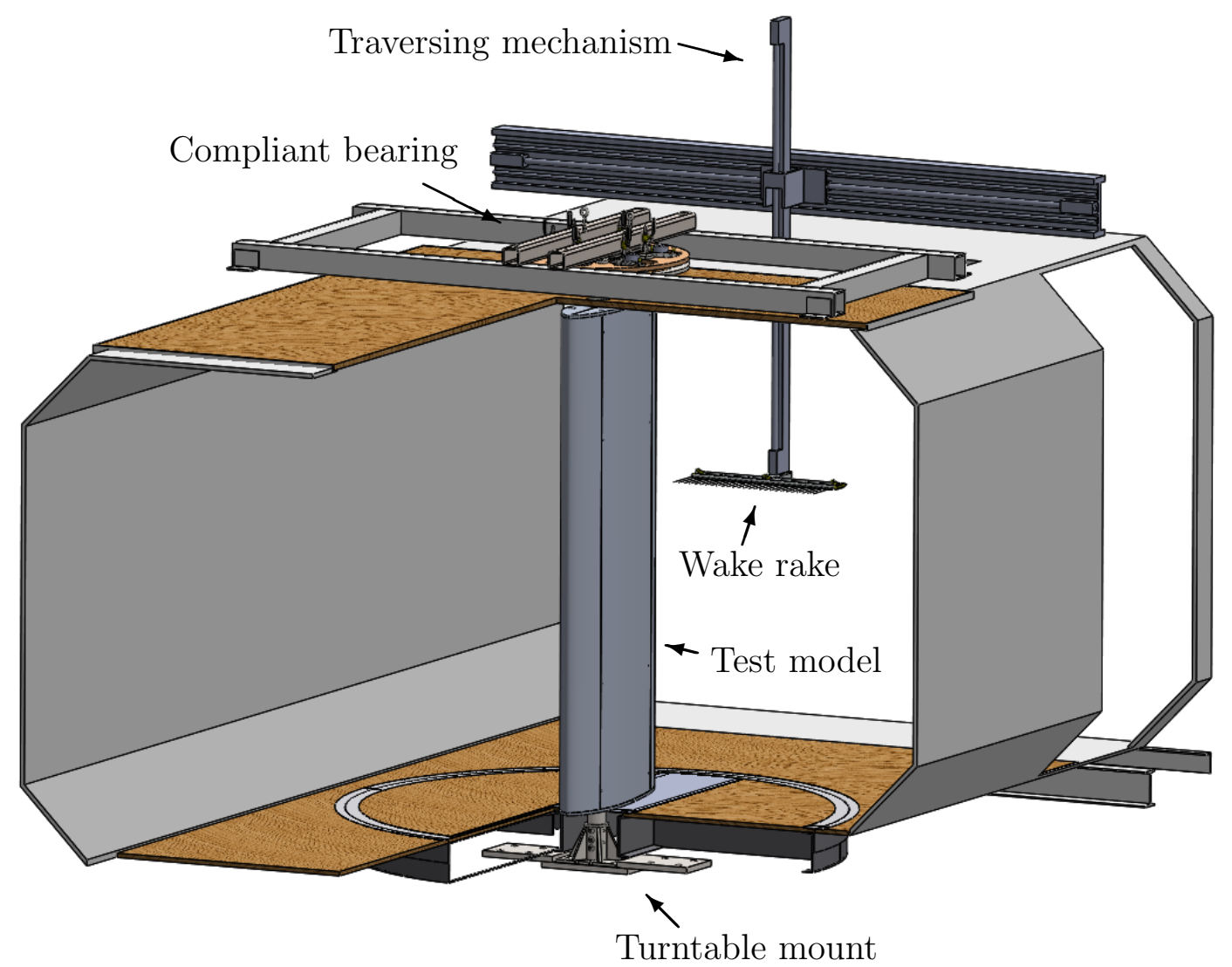

Figure III.1: Drawing of wind tunnel test section.

King's Law can be related to the wire voltage to arrive at the following equation [43]:

$$
E^{2}=A^{\prime}\left(T_{w}-T_{a}\right)+B^{\prime}\left(T_{w}-T_{a}\right) U^{1 / n^{\prime}},
$$

where $A, B$, and $n$ are calibration constants, $T_{w}$ is the wire temperature, and $T_{a}$ is the ambient flow temperature. Ambient flow temperature variations can be accounted for with a temperature-compensation coefficient if calibrations are performed at two flow temperatures. The calibration equations given by Equation 3.1 for hot and cold calibration temperatures can be subtracted and rearranged to yield this temperature- 
calibration constant, $C_{T}$ :

$$
C_{T}(U)=\left(E_{h}^{2}-E_{c}^{2}\right) /\left(T_{h}-T_{c}\right)=-A^{\prime}-B^{\prime} U^{1 / n^{\prime}}
$$

Voltages and flow temperatures for each calibration run are given by $E$ and $T$, respectively. The $h$ and $c$ subscripts refer to the hot and cold calibration runs. Temperature dependencies in Equation 3.1 can be removed by substituting the measured hotwire voltage with a compensated voltage given by $E_{\text {comp }}^{2}=E^{2}+C_{T}(U)\left(T_{\text {comp }}-T_{a}\right)$, where $T_{\text {comp }}$ is an arbitrary compensation temperature and $E_{\text {comp }}$ is the corresponding compensation voltage. Use of Equation 3.2 allows all flow temperature dependencies to be removed from Equation 3.1. Constant-temperature anemomters operate on a feedback loop which holds the wire temperature constant. Since wire and compensated temperatures are both constant, they can be absorbed into the calibration coefficients. The final calibration equation is written in terms of the flow velocity, compensated voltage, and three new calibration constants:

$$
U=\left(A+B E_{\text {comp }}^{2}\right)^{n}
$$

Three hotwires were attached to the traversing mechanism and calibrated using the procedure outlined above. The hotwires were positioned evenly in the vertical direction and spaced approximately 2 inches apart in the horizontal direction. Comparisons between the hotwires were used to verify repeatability of the results. Cold calibration occurred at the beginning of the flow quality tests when the temperature inside the tunnel was approximately $70^{\circ} \mathrm{F}$. Hot calibration occurred at the end of the tests when tunnel temperature was over $100^{\circ} \mathrm{F}$. The tunnel is not temperature 
controlled, so continuous running tends to heat up the tunnel. Dynamic pressure varied during the calibration from 5 psf to 75 psf in 5 psf increments. Results from the calibration are given in Figures III.2 and III.3.

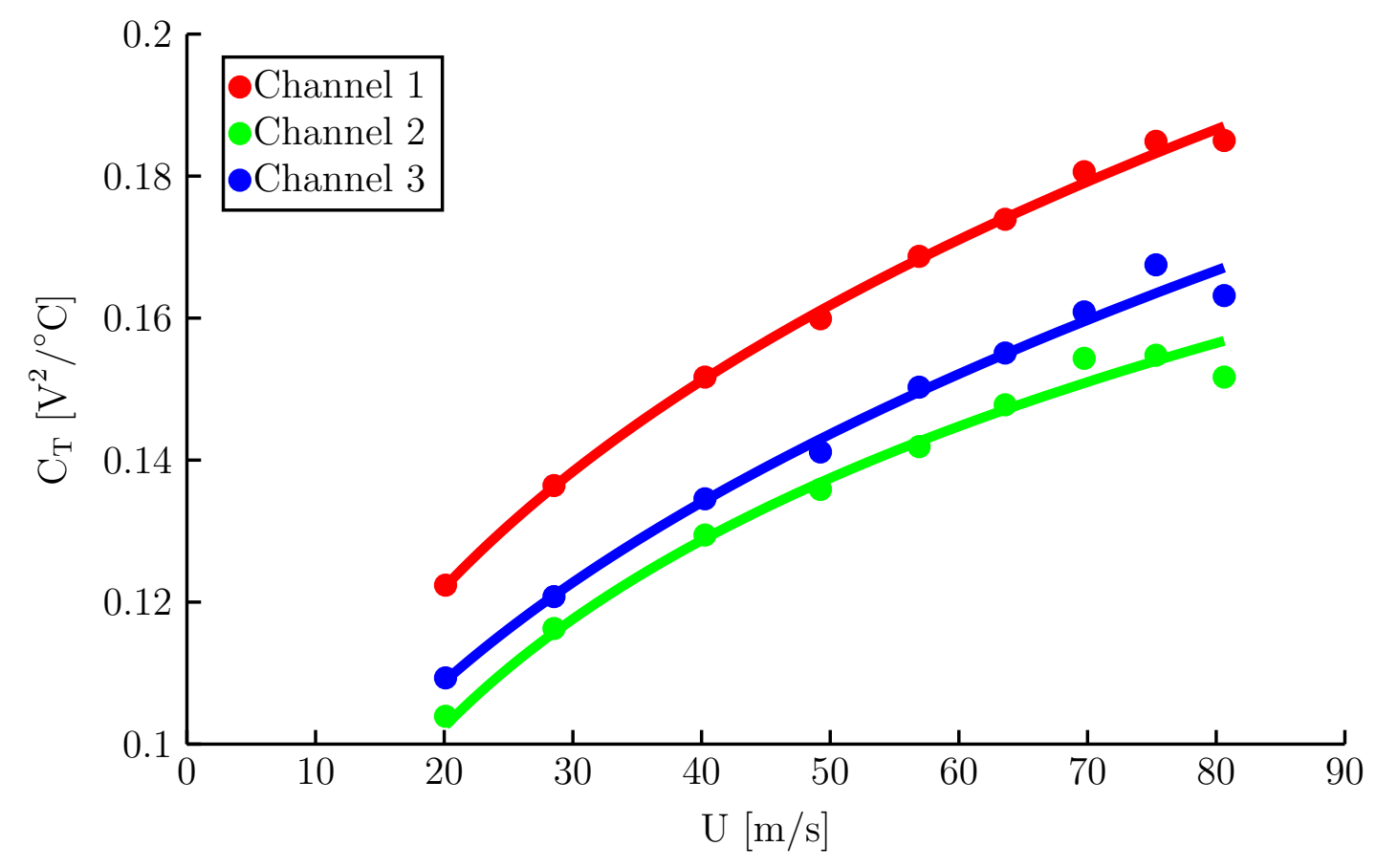

Figure III.2: Hotwire temperature compensation plots.

Once calibration was completed, the hotwires were moved through the tunnel in a 60 inch by 40 inch grid in 5 inch increments. This test was repeated four times at constant tunnel velocities corresponding to Reynolds numbers of $1.6 \times 10^{6}, 2.4 \times 10^{6}$, $3.2 \times 10^{6}$, and $4.0 \times 10^{6}$ for a 32 inch reference length. These are the same Reynolds numbers which were used during the main wind tunnel testing discussed in later sections. Hotwire voltages were sampled at $1 \mathrm{kHz}$ for 1 second at each measurement location. Results for flow uniformity at $R e_{c}=3.2 \times 10^{6}$ are given in III.4 as percent difference in velocity measured by the hotwires to velocity measured by the tunnel's 


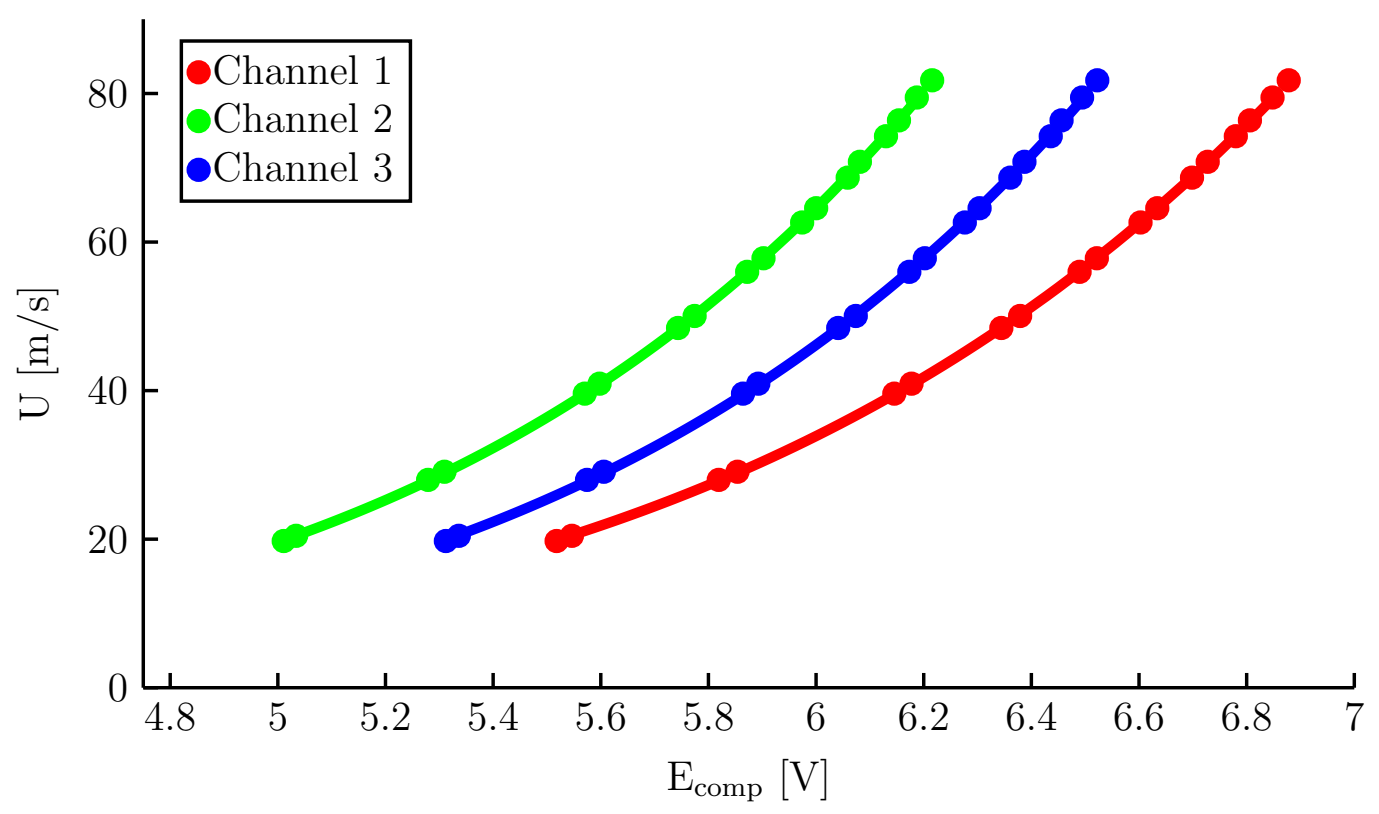

Figure III.3: Hotwire calibration plots.

pressure taps. These quantities are averaged over all three hotwires. Results for other Reynolds numbers are given in Appendix B. Flow uniformity is within approximately $1 \%$ throughout the tunnel at all conditions. Turbulence intensity was calculated at the center of the tunnel by computing the RMS velocities of the hotwire time-series data. Turbulence intensity is approximately $0.8 \%$ for all Reynolds numbers between $1.6 \times 10^{6}$ and $4.0 \times 10^{6}$.

III.B. Model Construction

A hollow, aluminum model of the NREL S814 was designed to mount vertically inside the 7 -ft-tall by 10-ft-wide LSWT test section. Model requirements included a large enough chord length to achieve the desired Reynolds numbers, large regions of undisturbed boundary layer flow, and easy access to internal instrumentation. 


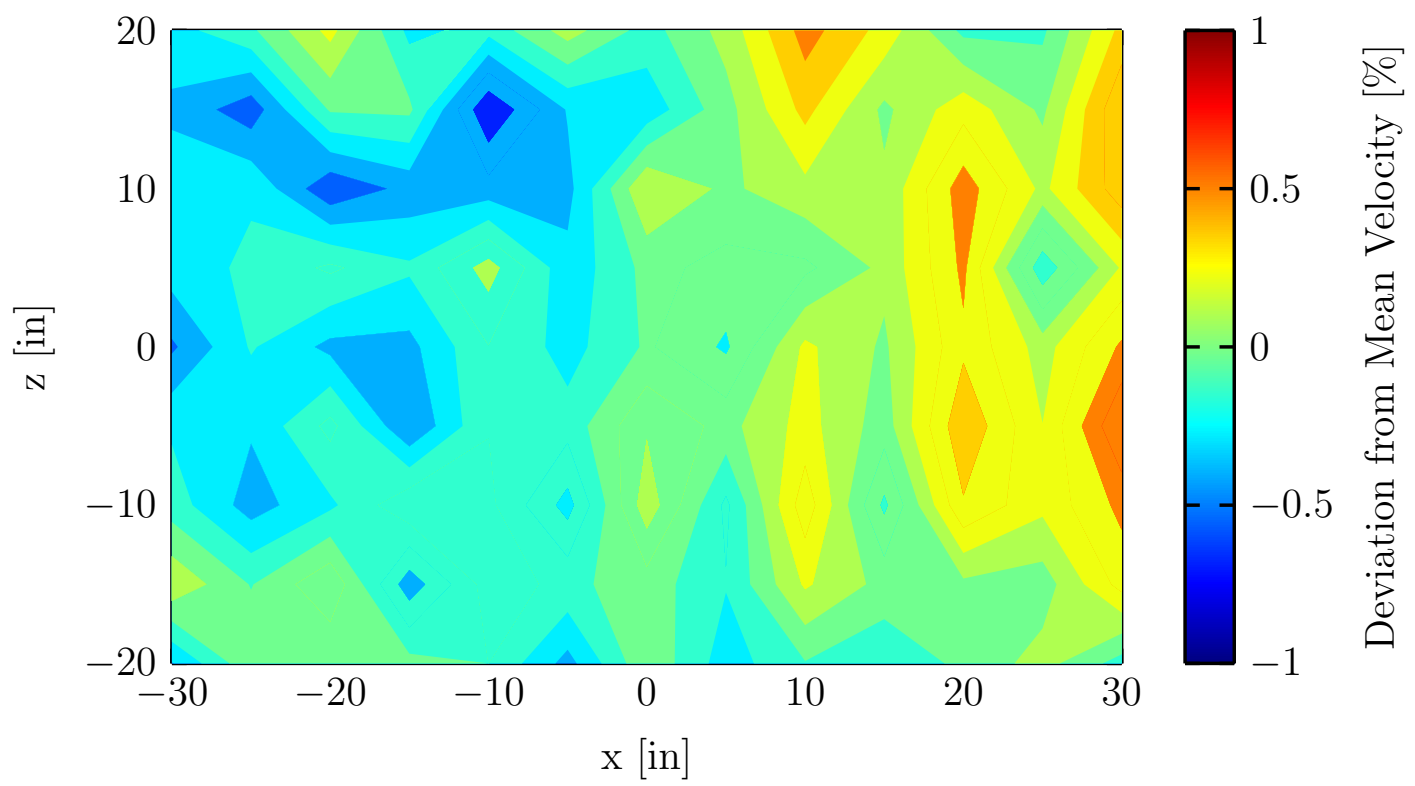

Figure III.4: Flow uniformity plot at $R e_{c}=3.2 \times 10^{6}$.

Special care was also taken to ensure that the model is amenable to testing using pressure and IR measurements. Initial airfoil profile coordinates were taken from the University of Illinois Urbana-Champaign (UIUC) airfoil database. This profile was then thickened, while maintaining camber, at locations aft of $90 \%$ chord for ease of manufacturing.

The outer mold line of the airfoil is separated into four distinct regions: upper surface, lower surface, leading edge, and trailing edge. Each of these sections spans 6 feet and 11.75 inches and has a constant wall thickness of 0.5 inches. A chord length of 32 inches was chosen to match that of Ehrmann's NACA $63_{3}-418$ model, resulting in $6.5 \%$ blockage. The model mounts into the wind tunnel via a hollow steel shaft (3 inch outer diameter, 2.5 inch inner diameter). The lower shaft connects through the wind tunnel floor to the tunnel's external balance and turntable assembly which controls the model angle of attack. The upper shaft connects to a compliant 
bearing assembly which restricts planar movement but allows pitching of the model [7]. The bearing also shares a portion of the load from the model which prevents the wind tunnel balance from overloading. Four mount assemblies provide connections between the shaft and the model's interior.

The main structural elements inside the model are four, 1-inch-thick spars. The spars connect the shaft mount assemblies, upper surface, and lower surface. Tests on the NACA $63_{3}-418$ model revealed that internal spars can act as heat sinks, causing the airfoil surface to heat unevenly and contaminating IR data [44]. The NREL S814 model design combats this by extending the spars only along the bottom and top quarters of the model. This ensures that the middle half of the model has a consistent thermal response. The internal spar placement also allows for easy access to internal pressure tubing which is connected to pressure taps on the model surface. An image of the wind tunnel assembly is given in Figure III.5.

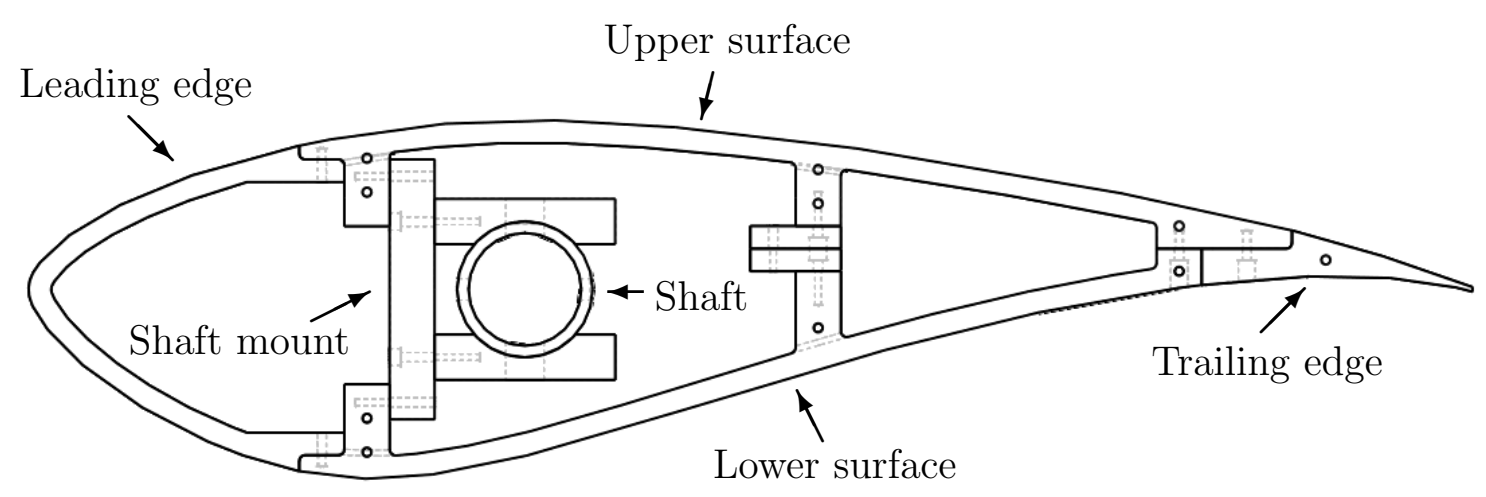

Figure III.5: Drawing of NREL S814 wind tunnel model.

Model pieces were machined using a CNC mill. During fabrication, a rough machining pass was made to define the general shape and features of each model piece. 
The upper surface, leading edge, and trailing edge were then assembled together before a final machining pass was conducted to eliminate steps and gaps between pieces. This process was repeated using the lower surface, leading edge, and trailing edge. The model was assembled using a combination of 0.25 inch bolts and dowel pins. With the exception of four bolts and four pins along the model's trailing edge, no connectors are exposed to the flow. Two sets of endplates (not shown in Figure III.5) allow access to the internal connections.

A hammer drill, 0.040 inch drill bit, and rapid-prototyped drill guide were used to drill 54 pressure taps into the model. Hole spacing was chosen so that 28 taps were equally spaced in the first $30 \%$ of chord and the remainder were equally spaced along the back $70 \%$ chord. Appendix C contains the pressure tap coordinates. Stainless steel, 0.040 inch, Scanivalve tubulations were installed in each tap and connected to urethane tubing which was run outside the model through a hole in the upper endplate. Finally, the model was painted with a $250 \mu \mathrm{m}$ layer of Sherwin-Williams ${ }^{\circledR}$ lusterless high solids polyurethane topcoat. The flat surface finish prevents surface reflections, and the thick coat acts as thermal insulation which improves IR thermography analysis.

\section{III.C. Roughness Modeling}

Trip strips and sand grain roughness are typically used to test an airfoil's sensitivity to roughness. However, research has shown that these methods are unrepresentative of real insect roughness. The most realistic approaches to insect roughness

modeling were done in tests conducted by Moroz \& Eggleston [8] and Coleman [11]. 
In Moroz \& Eggleston's tests a mold was created of actual insect contamination patterns whereas in Coleman's tests actual insects were taken from airfoils and applied directly to the model. These methods are extremely realistic but prohibitively costly. Instead, a method of using vinyl decals to simulate insects was chosen. Decals are an inexpensive insect modeling solution which should closely replicate actual contamination. Furthermore, the effect of roughness height and amount of roughness can easily be studied using decals. One of the few disadvantages of insect decals is their low thickness-to-diameter ratios $(k / d)$. Krishnan showed that insect contamination very near the leading edge is composed mainly of insects with $k / d$ values near unity [40]. At more downstream locations, insects impact the blade less directly and are consequently smeared over the surface. For this study, $k / d$ values are more typical of these smeared insects.

Insect decals are created by generating a random pattern of circles $2.4 \mathrm{~mm}$ in diameter inside of a rectangular grid. The size of the grid was determined through the concept of "roughness region". The roughness region is the region in which insect collection is highest. This region is expected to contain the largest insects as well as the highest insect percent-area-coverage. LEWBUG results were used with a $30 \%$ collection efficiency cutoff to determine the size of the roughness region. This cutoff is approximately equivalent to the rupture velocity cutoff at the spanwise location of maximum power production for the NREL 5 MW turbine discussed in Section II.C. The roughness region for the NREL S814 airfoil extended from $s / c=0.045$ on the upper surface to $s / c=0.192$ on the lower surface. The roughness region concept was also utilized in Ehrmann's tests, resulting in a smaller roughness region from 
$s / c=0.045$ to $s / c=0.125$. Figure III.6 illustrates the roughness regions for each airfoil.

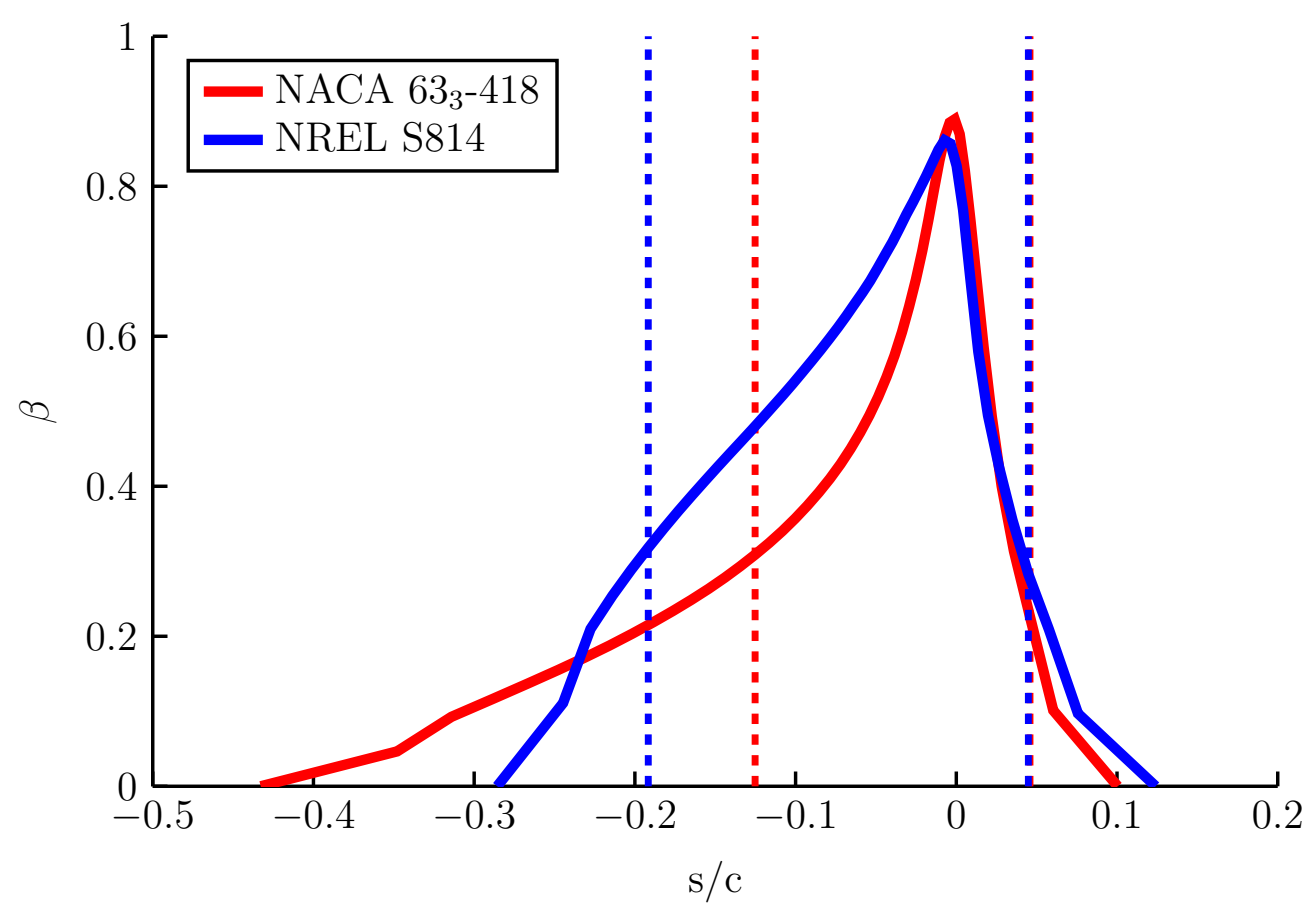

Figure III.6: Roughness region comparison. The $s / c$ locations within the dashed lines correspond to the roughness regions for each airfoil.

Seven roughness configurations were tested for each airfoil. Each roughness configuration is comprised of a roughness density (percent-area-coverage) and roughness height. Roughness height was varied by using different vinyl thicknesses. Roughness density was varied by changing the number of circles contained within each roughness pattern. Inside the roughness region, roughness density was kept constant. In actuality, density decreases with distance from the leading edge. However, keeping a constant density allows this test method to be more easily adapted to other wind tunnel experiments. Furthermore, early wind tunnel results from 
Ehrmann showed that density variation is less important than height variation [7]. One change from Ehrmann's experiment is that ellipsoidal roughness elements are not tested. Ehrmann showed that the airfoil performance difference in using all circular elements versus a combination of circular and ellipsoidal elements is negligible relative to performance differences due to varying roughness height and density. Two example roughness patterns of varying density are shown Figure III.7. These patterns were cut from vinyl sheets and applied to the model. The red triangles were used for alignment of the pattern on the model and were removed prior to testing.

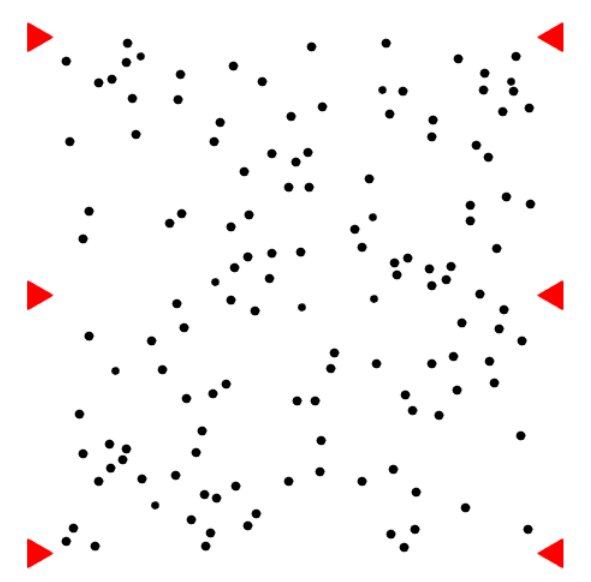

a)

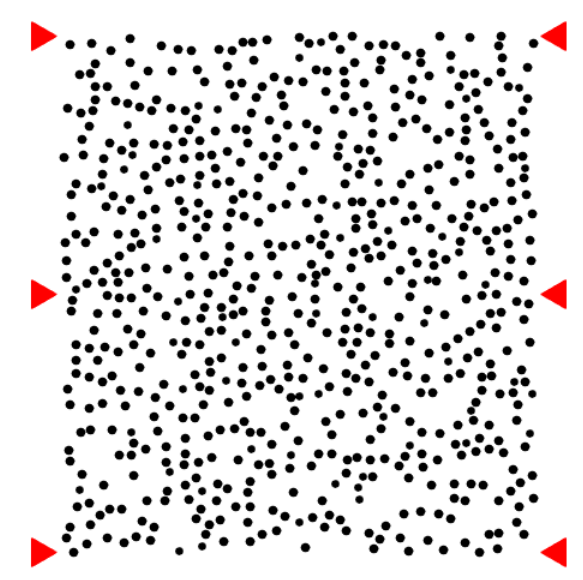

b)

Figure III.7: Insect roughness patterns. Patterns correspond to (a) 3\% density and (b) $15 \%$ roughness density.

In addition to roughness decals, a clean configuration and trip strip configuration were tested. The trip strip configuration is used for comparison with historical methods of studying roughness sensitivity. The trip strip is located at $2 \%$ chord $(x / c)$ on the upper surface and $10 \%$ chord on the lower surface and has a $60^{\circ}$ zigzag pattern with $6 \mathrm{~mm}$ wavelength, $9 \mathrm{~mm}$ peak-to-peak amplitude, and a nominal height 
of $500 \mu \mathrm{m}$. At high Reynolds numbers the large tape height may result in early separation. However, this thickness is necessary to properly trip the boundary layer at low Reynolds numbers. The trip strip is used for comparison only, so a single height was deemed appropriate for all Reynolds numbers.

\section{III.D. Testing Procedure}

Wind tunnel testing was split into two test entries. Each entry was composed of many runs, each consisting of an angle-of-attack sweep in $1^{\circ}$ increments at a set Reynolds number. Pressure and infrared (IR) measurements were made at each angle of attack. Reynolds numbers varied between $1.6 \times 10^{6}, 2.4 \times 10^{6}, 3.2 \times 10^{6}, 4.0 \times 10^{6}$ for all runs. Angle of attack was varied between $-16^{\circ}$ and $+16^{\circ}$ for the three lowest Reynolds numbers. At $R e_{c}=4.0 \times 10^{6}$ the angle-of-attack range was limited to $-5^{\circ}$ to $+5^{\circ}$ to avoid overloading the LSWT external balance. Balance data was not collected during the test, but excessive forces were avoided to prevent damage to the balance structure. Instead, lift and pitching were determined through surface pressure measurements, and drag was determined by wake pressure measurements using a 28 port wake rake. At each angle of attack, the Reynolds number was held within a tolerance of 20,000 for 10 seconds. Pressure data was sampled over this period at a frequency of $1 \mathrm{kHz}$, averaged, and written to output files containing tunnel flow conditions, angle of attack, and wake rake position. Transition images were recorded manually for each angle of attack using IR thermography.

The first entry was used primarily as testing to determine areas in which the

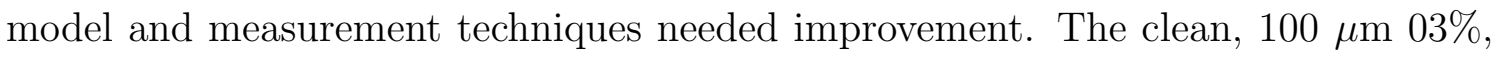


$140 \mu \mathrm{m} \mathrm{03 \%}$, and $200 \mu \mathrm{m} 03 \%$ roughness configurations were tested during this entry. MATLAB data analysis codes were written prior to the second entry such that the data analysis during the second entry could be conducted during the test. This allowed runs with bad data points due to instrument malfunctions or user errors to be repeated. The second test entry contained the entire range of test configurations. All data presented in this report comes from the second test entry.

\section{III.E. Test Instrumentation}

The LSWT contains its own data acquisition hardware for measurements of freestream conditions, model surface pressures, and wake rake pressures. Freestream total and static pressures were measured using a Pitot-static probe located at the test section inlet. Barometric pressure measurements were taken in the LSWT balance room with an uncertainty of $\pm 11.5 \mathrm{~Pa}$. Model surface pressures were read with a 64 -port pressure scanner with a range of \pm 5 psi. Wake rake pressures were read with a 32-port pressure scanner with a range of \pm 1 psi. Accuracies for the 64 -port and 32-port scanners are $\pm 17 \mathrm{~Pa}$ and $\pm 7 \mathrm{~Pa}$, respectively. Both scanners, as well as tunnel static pressure measurements, measure differential pressures referenced to the static pressure in the balance room. Voltages from these scanners were acquired by the LSWT data system. Pressure readings were found to drift due to tunnel temperature variations. Technical specifications indicate up to $\pm 21 \mathrm{~Pa} /{ }^{\circ} \mathrm{C}$ and $\pm 7 \mathrm{~Pa} /{ }^{\circ} \mathrm{C}$ errors for the 64-port scanner due to offset and span variations, respectively, caused by temperature drift. For the 32 -port scanner, technical specifications show $\pm 14 \mathrm{~Pa} /{ }^{\circ} \mathrm{C}$ and $\pm 2 \mathrm{~Pa} /{ }^{\circ} \mathrm{C}$ offset and span errors, respectively. Voltage readings for zero tunnel 
velocity were taken from each scanner at the beginning and end of each of test run and used to calibrate a linear temperature versus voltage relationship for each scanner. This was accomplished automatically through LSWT codes before any other data processing began.

The wake rake consisted of 25 Pitot probes and three static probes. Output from the three static probes was found to be unreliable, so tunnel static pressure measured at the test section inlet was used instead. The wake rake was placed $0.9 c$ downstream of the wing trailing edge. Barlow suggests a minimum distance of $0.7 c$ so that wake measurements are not affected by static pressure variation near the wing [45]. Additionally, Ehrmann found that flow over model pressure ports can lead to a turbulent wake downstream of the ports' spanwise locations. To avoid these areas, the wake rake was placed well below the pressure ports approximately 2 feet from the tunnel floor. This position avoids turbulent spreading from both the floor and pressure ports.

Two IR cameras were used to view the model surface, a FLIR ${ }^{\circledR}$ SC8000 on the lower surface and a ThermaCAM ${ }^{\circledR}$ SC3000 on the upper surface. The SC8000 has a $1,024 \times 1,024$ resolution and operates within a $-20^{\circ} \mathrm{C}$ to $50^{\circ} \mathrm{C}$ range with an accuracy of $\pm 2^{\circ} \mathrm{C}$. The SC3000 produces a $320 \times 240$ resolution image and has an operating range of $-15^{\circ} \mathrm{C}$ to $50^{\circ} \mathrm{C}$. Accuracy is $\pm 1^{\circ} \mathrm{C}$. The test section plexiglass windows in the LSWT are not transmissive in the IR range. These windows were removed prior to testing and replaced with two plywood panels with sealed pressure boxes to house the cameras. Holes were cut into each panel so that the model was viewable from cameras mounted inside the boxes. Mounting the cameras in 
these boxes reduced flow disturbances due to flow leakage, but this leakage is likely minimal since the test section is vented to atmospheric pressure. IR measurements were acquired on a computer using ExaminIR ${ }^{\circledR}$ and ThermaCAM $^{\circledR}$ software. This computer was connected to the cameras via USB, and accessed via the LSWT control using remote desktop.

\section{III.F. Lift, Pitching Moment, and Drag Measurements}

Lift and pitching moment were found by integration of the model's surface pressure distribution. Surface pressures measured by the 56 pressure taps were first cast into the dimensionless pressure coefficients:

$$
c_{p}=\frac{p-p_{\infty}}{q}
$$

where $c_{p}$ is the pressure coefficient, $p$ is the surface pressure at each pressure tap location, and $p_{\infty}$ and $q$ are the freestream static pressure and dynamic pressure measured at the test section inlet.

The small thickness of the trailing edge made installing a pressure port in this location impossible. Instead, the average of the furthest downstream ports on the upper and lower surfaces was used to specify a pressure coefficient at the trailing edge. Example pressure coefficient plots are given in Figure III.8.

Pressure coefficients were integrated around the model surface to determine the axial and normal force coefficients acting on the airfoil:

$$
c_{n}=-\oint c_{p} d\left(\frac{x}{c}\right)
$$



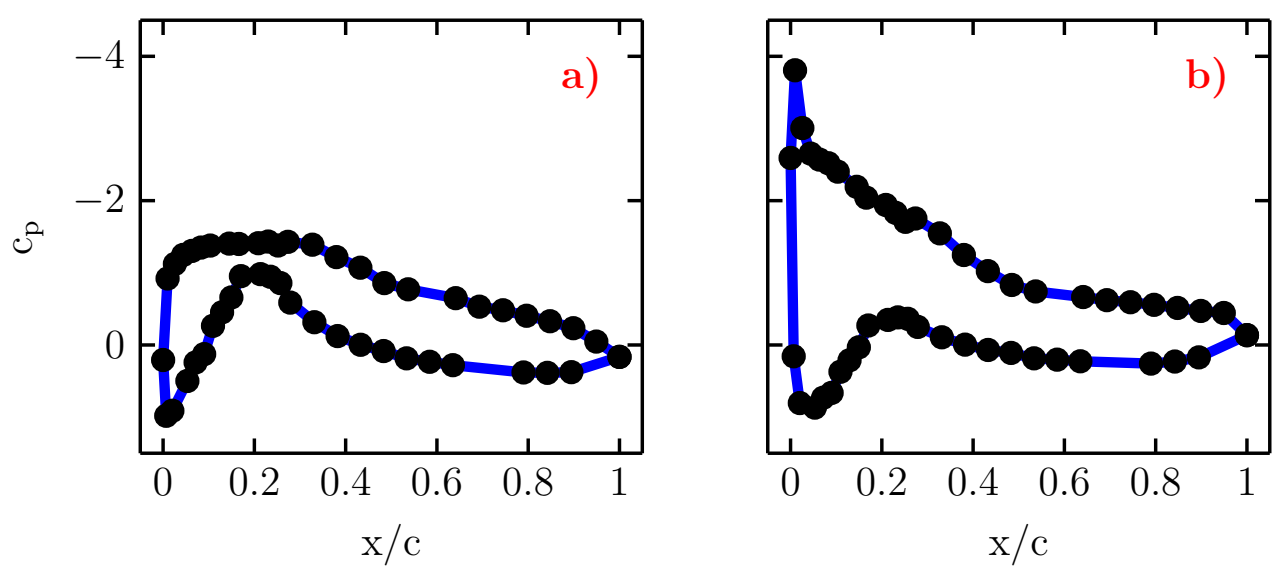

Figure III.8: Example pressure coefficient plots. Plots correspond to the NREL S814 clean configuration at $R e_{c}=3.2 \times 10^{6}$ for (a) an angle of attack of $4^{\circ}$ and (b) an angle of attack of $13^{\circ}$.

$$
c_{a}=+\oint c_{p} d\left(\frac{y}{c}\right)
$$

where $c_{n}$ and $c_{a}$ are the normal and axial force coefficients, respectively. The integrations are performed from the lower surface trailing edge to the upper surface trailing edge.

Converting $c_{n}$ and $c_{a}$ to lift coefficient requires a coordinate transformation into the wind frame:

$$
c_{l}=c_{n} \cos (\alpha)-c_{a} \sin (\alpha)
$$

where $c_{l}$ is the airfoil lift coefficient and $\alpha$ is the angle of attack. The axial coefficient term is typically ignored since both $\sin (\alpha)$ and $c_{a}$ are small compared to the other terms. These terms become important at high angles of attack and stalled conditions, so they were retained to increase accuracy for these conditions.

Pitching moment coefficient can be found through direct integration of the pressure coefficients since pitching moment is identical in both the model and wind 
frames:

$$
c_{m, c / 4}=\oint c_{p}\left(\frac{x}{c}-0.25\right) d\left(\frac{x}{c}\right)+\oint c_{p}\left(\frac{y}{c}\right) d\left(\frac{y}{c}\right) .
$$

The pitching moment coefficient $c_{m, c / 4}$ is written about the airfoil quarter-chord, and a positive value is used for a nose-up pitch.

The normal and axial force components can also be used to define the drag coefficient:

$$
c_{d}=c_{n} \sin (\alpha)+c_{a} \cos (\alpha),
$$

where $c_{d}$ is the airfoil drag coefficient. However, drag coefficients are typically multiple orders of magnitude less than lift coefficients. Measurement error of surface pressures make this approach unreliable for drag measurements. More importantly, this method does not account for skin-friction drag which is a large component of the total drag.

A control volume approach using the wake data can capture all types of drag and reduces uncertainty due to surface integrations. This approach was used here. The drag is related to the velocity deficit behind the airfoil through Equation 3.10:

$$
D^{\prime}=\int \rho U\left(U_{0}-U\right) d y
$$

where $D^{\prime}$ is the drag per unit span, $\rho$ is the flow density, $U_{0}$ is the velocity upstream of the airfoil, and $U$ is the velocity in the wake.

Drag can be nondimensionalized by dividing by dynamic pressure and chord:

$$
c_{d}=2 \int\left(\sqrt{\frac{q}{q_{0}}}-\frac{q}{q_{0}}\right) d\left(\frac{y}{c}\right)
$$


where $q$ is the dynamic pressure in the wake and $q_{0}$ is the upstream dynamic pressure. In unbounded flows, the integrand disappears outside the wake. Tunnel walls, however, constrain the flow so that the dynamic pressure outside the wake is slightly increased from the upstream dynamic pressure in order to preserve mass continuity. Due to circulation produced by the model, the dynamic pressure outside the wake is also not constant. To correct for both of these effects, a linear fit is applied to the local dynamic pressures outside the wake. The pressures given by this fit replace $q_{0}$ in Equation 3.11, causing the integrand to disappear outside the wake as intended. An example wake profile after this fit has been applied is shown in Figure III.9 for two angles of attack. The increased size of the wake for separated flows is evident by comparing the two figures.
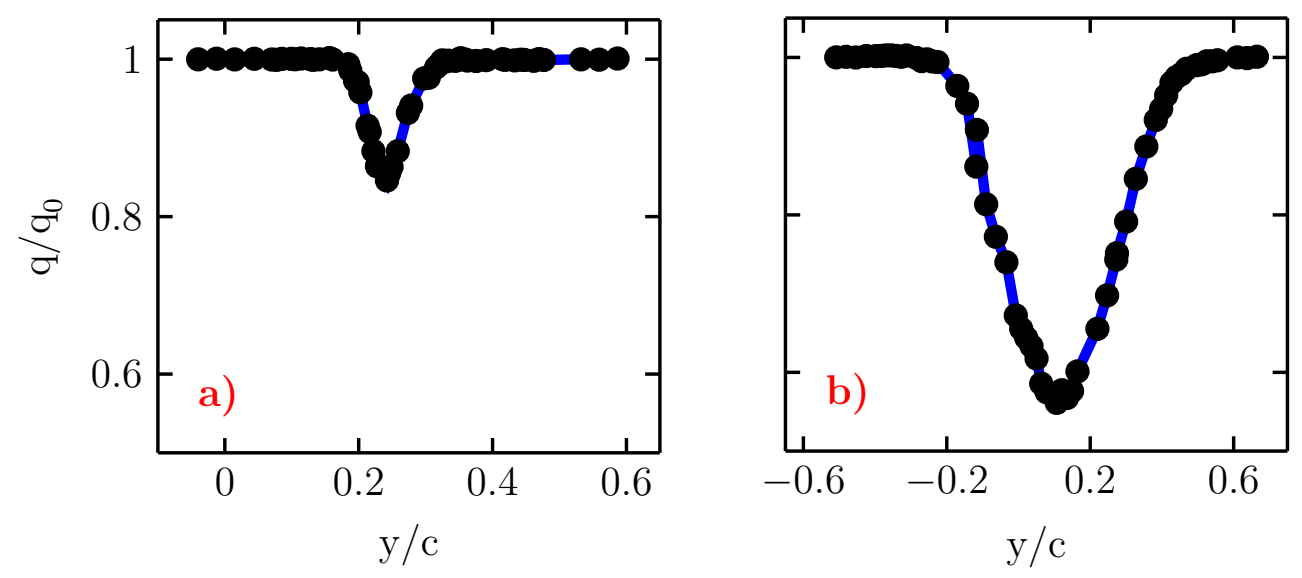

Figure III.9: Example wake plots. Plots correspond to the NREL S814 clean configuration at $R e_{c}=3.2 \times 10^{6}$ for (a) an angle of attack of $4^{\circ}$ and (b) an angle of attack of $13^{\circ}$.

For separated flows, the wake becomes larger than the span of the wake rake so that capturing the entire wake in one measurement is impossible. Instead, three 
wake measurements at different lateral locations were taken for each test point. The center of the wake rake for these points was positioned at the left side, the right side, and the center of the wake deficit. These locations were determined from diagnostic test runs for each configuration. For attached flow, the wake is small and capable of being captured by a single wake measurement. However, three wake locations were still taken for these flows in order to increase measurement resolution. Results from the first test entry showed that unsteadiness for separated flows could cause the wake structure to vary at each measurement location. This issue was corrected in the second test entry by increasing sampling time for each point from 1 second to 10 seconds.

Uncertainties in tunnel dynamic pressure, airfoil surface pressures, and wake pressures lead to uncertainty in lift, drag, and pitching moment. These were calculated using Monte Carlo simulations on the clean configuration results. To perform each iteration of the simulations, pressures were randomly chosen from a normal distribution with mean values and standard deviations given by the experimentally measured values and their uncertainties. Each Monte Carlo simulation contain 1,000 iterations for each experimental test point. The uncertainties in airfoil performance coefficients was calculated as the standard deviation of the resulting lift, drag, and pitching moment distributions, where each data point in the distribution corresponds to a different iteration point. 


\section{III.G. Wind Tunnel Corrections}

Wind tunnel testing introduces factors which are not present in atmospheric tests. Flow direction, velocities, and forces are all influenced by wind tunnel walls and mounting procedures, and these influences must be calibrated out of the final results using correction procedures.

Alignment of the model chordline to the flow direction is difficult because blockage effects and circulation divert the freestream flow. Mechanical slop in mounting points and shaft deflections further increase the difficulty. Rather than attempting to align the model exactly, angle-of-attack corrections were applied to the final results. First, viscous simulations using XFOIL were run for each Reynolds number to determine the expected zero-lift angle of attack. Experimental zero-lift angles of attack were determined through linear interpolation of the clean configuration experimental data at each Reynolds number. Roughness can cause shifts in zero-lift angle of attack, so only clean data was utilized at this step. The angle-of-attack shifts between the experimental and simulated data for each Reynolds number were determined, and the experimental angles of attack were adjusted by this amount. The correction amount was allowed to vary for different Reynolds numbers since higher loads can result in additional twisting of the airfoil. For the first test entry, the largest shifts were over $2^{\circ}$. Mounting hardware was tightened during the second entry and the shift was reduced to less than $0.5^{\circ}$.

Wall corrections were performed using the method described in Barlow [45]. These corrections account for solid blockage, wake blockage, and streamline cur- 
vature. The wind tunnel walls diverge slightly to compensate for boundary layer growth, making bouyancy corrections unnecessary.

Solid blockage corrections account for the additional velocity around the model due to the model's size. Streamlines are constrained within the test section walls, so velocity must increase around the model to satisfy mass continuity. The solid blockage correction term is related to the model size through

$$
\epsilon_{s b}=\Lambda \sigma,
$$

where $\epsilon_{s b}$ is the wake blockage correction term, $\Lambda$ is related to the model's volume, and $\sigma=(\pi c / h)^{2} / 48 \approx 0.0146$, where $c$ is the chord length and $h$ is the tunnel height for horizontally-mounted models or width for vertically-mounted models. $\Lambda$ is not tabulated for a NREL S814 airfoil, but Selig shows that the correction is proportional to model volume [46]. Therefore, the tabulated value for the NACA $63_{3}-018$ of $\Lambda \approx 0.316$ was adjusted proportionally to the airfoil's volume ratio to arrive at $\Lambda \approx 0.42$ for the NREL S814. Because the corrections are small, this approximation does not introduce significant uncertainty.

The second blockage component, wake blockage, is proportional to airfoil drag:

$$
\epsilon_{w b}=\frac{c}{2 h} c_{d u},
$$

where $\epsilon_{w b}$ is the wake blockage correcterion term and $c_{d u}$ is the uncorrected drag coefficient. Wake blockage accounts for the increased velocity of the flow outside the wake in order to preserve mass continuity. This is the reason that the freestream dynamic pressure downstream of the model is slightly increased from its upstream 
value as mentioned in Section III.F. The total blockage correction is the sum of solid and wake blockage corrections and represents the combined effect of accelerated flow around the model:

$$
\epsilon=\epsilon_{s b}+\epsilon_{w b}
$$

A final correction is applied to account for streamline curvature. An airfoil producing lift creates circulation which can be modeled as a vortex bound to the airfoil. In the wind tunnel, the walls give rise to mirror vortices which cause streamlines to curve and modify the airfoil's angle of attack, lift, and pitching moment. The amount of streamline curvature is related to the lift and pitching moment produced by the airfoil. The final correction equations which account for blockage and streamline curvature are given below:

$$
\begin{gathered}
q=q_{u}(1+2 \epsilon), \\
c_{d}=c_{d u}\left(1-3 \epsilon_{s b}-2 \epsilon_{w b}\right), \\
\alpha=\alpha_{u}+\frac{\sigma}{2 \pi}\left(c_{l u}+4 c_{m u, c / 4}\right), \\
c_{l}=c_{L u}(1-\sigma-2 \epsilon), \\
c_{m, c / 4}=c_{m u, c / 4}(1-2 \epsilon)+\sigma c_{L} / 4,
\end{gathered}
$$

The subscripts $u$ in these equations refer to the uncorrected coefficients. The corrected performance coefficients are output to text files and used later to estimate annual energy production. 


\section{III.H. Transition Location Measurements}

IR thermography uses differences in convective heat transfer rates between laminar and turbulent flows to provide visualizations of transition locations. Turbulent flows have heat transfer coefficients which are typically an order of magnitude greater than those of laminar flows. The LSWT does not have a temperature control system, so the tunnel naturally heats up during test operations. The large thermal mass of the aluminum model means that the model typically lags behind the tunnel temperature by a few degrees. Portions of a model over which air is turbulent will therefore heat up much quicker than portions with laminar flow. Temperature changes between these two flow regimes are evident in IR images of the model surface in which turbulent areas are brighter than laminar areas.

Before testing began, a grid was drawn onto both the upper and lower surfaces of the airfoil with a metallic silver marker. The grid location was such that the boundary layer over this area was undisturbed by turbulent from walls and pressure taps. A turbulence spreading angle of $15^{\circ}$ from these features was assumed to ensure that this was true. Grid vertices were spaced 4 inches apart, measured with a flexible ruler along the airfoil surface beginning at the trailing edge. The metallic silver color of the marker is highly reflective and appears on IR images, making the vertices ideal as fiducial markers. The IR cameras were positioned on adjustable mounts in the pressure box and aligned such that the grid was in full view. Once a proper camera position was determined, the cameras were locked into place for the entirety of wind tunnel testing. Once locked, reference images with no air flow were recorded at each angle of attack as the model was swept through an angle-of-attack range 
from $-16^{\circ}$ to $+16^{\circ}$ in $1^{\circ}$ increments. Fiducial markers are clearer in these images than in the other test images and are used later to convert image pixel locations to airfoil coordinates.

During testing, images were taken for each angle of attack. Two transition types were evident from the images: TS-dominated transition and bypass-dominated transition. TS-dominated transition occurs for the clean configuration and for certain angles of attack for the roughness configurations. It is characterized by a 2D transition front which varies gradually with angle of attack. Bypass transition occurs when the roughness becomes "critical" and immediately trips the boundary layer. Turbulent wedges form behind each critical roughness element and create a sawtooth pattern on the IR images. Figure III.10 shows examples of TS-dominated and bypass-dominated flows. Flow is from left to right. For angles of attack near the start of bypass, only a few wedges may be formed from the most critical roughness elements. The elements' chordwise position as well as slight variations in height contribute to making some elements more critical than others. Images and roughness elements were manually inspected after each test to ensure that premature bypass was not occurring due to decals peeling up from the surface. Tests in which this occurred were repeated. Once all data was collected, the angle of attack in which bypass first occurred for each configuration and Reynolds number combination was determined by sight. The formation of at least three turbulent wedges in the images was used as the criteria for judging when this occurred. Bypass angles of attack differed on the upper and lower surfaces of the airfoil. 

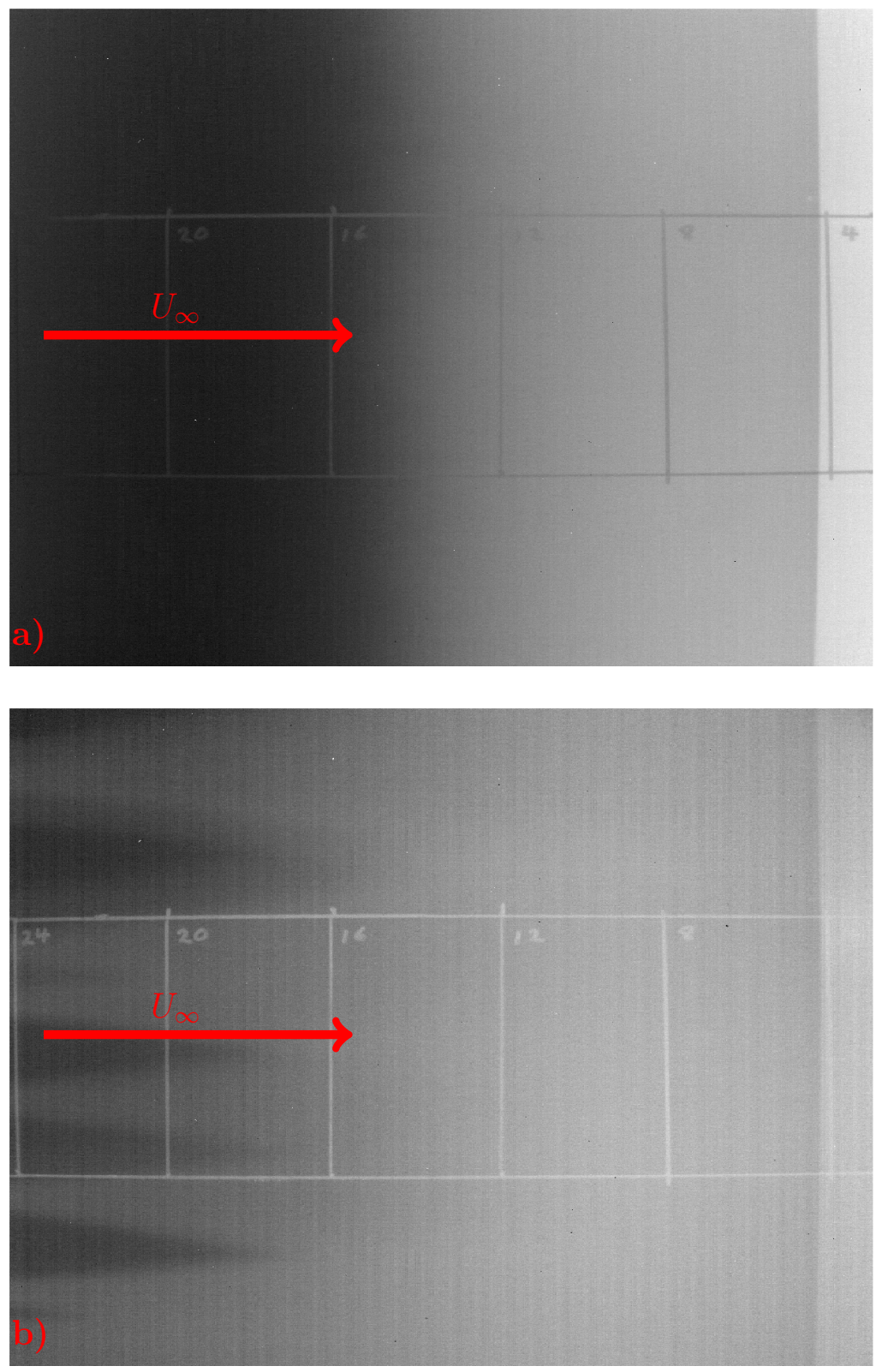

Figure III.10: Example infrared images. Images correspond to the NREL S814 airfoil at $R e_{c}=3.2 \times 10^{6}$ and an angle of attack of $-9^{\circ}$ for (a) the clean configuration (Tollmien-Schlichting transition) and (b) the $200 \mu \mathrm{m} 03 \%$ roughness configuration (bypass transition). 
For bypassed angles of attack, transition location was not analyzed further. The $3 \mathrm{D}$ behavior of the transition front for these flows makes determination of a 2D transition location less meaningful. For TS-dominated angles of attack, a MATLAB program was written to automatically determine the transition locations. The program performs spanwise averaging of pixel intensity values (proportional to temperature) of the IR images to generate average pixel intensities at each chordwise position. Small-scale pixel intensity fluctuations due to fiducial markers and measurement uncertainty are removed by boxcar-averaging the average pixel intensity values. Large-scale fluctuations are then quantified by taking the numerical derivative of the pixel intensities with respect to chordwise location. The areas in which the derivatives are highest correspond to the areas of highest temperature changes. Thus, the image location of maximum derivative is the transition location. For some angles of attack, the LSWT test section walls were captured in the images which are at a much different temperature than the model. Large thermal gradients are also observed at the interface between the main model surface and the trailing edge. These areas are excluded from the maximum derivative search to prevent the algorithm from erroneously selecting them as the transition locations. Figure III.11 shows the average pixel intensities and calculated transition location (in red) for the clean configuration at $R e_{c}=3.2 \times 10^{6}$ and an angle of attack of $-9^{\circ}$. The trailing edge is evident in the plot as a large decrease in $d I / d x$. Figure III.12 is an IR image of the model's upper surface with the calculated transition location marked in red.

The reference (no-flow) images of the fiducial markers for each angle of attack are utilized next. First, the known $s / c$ locations of the fiducial markers are converted 

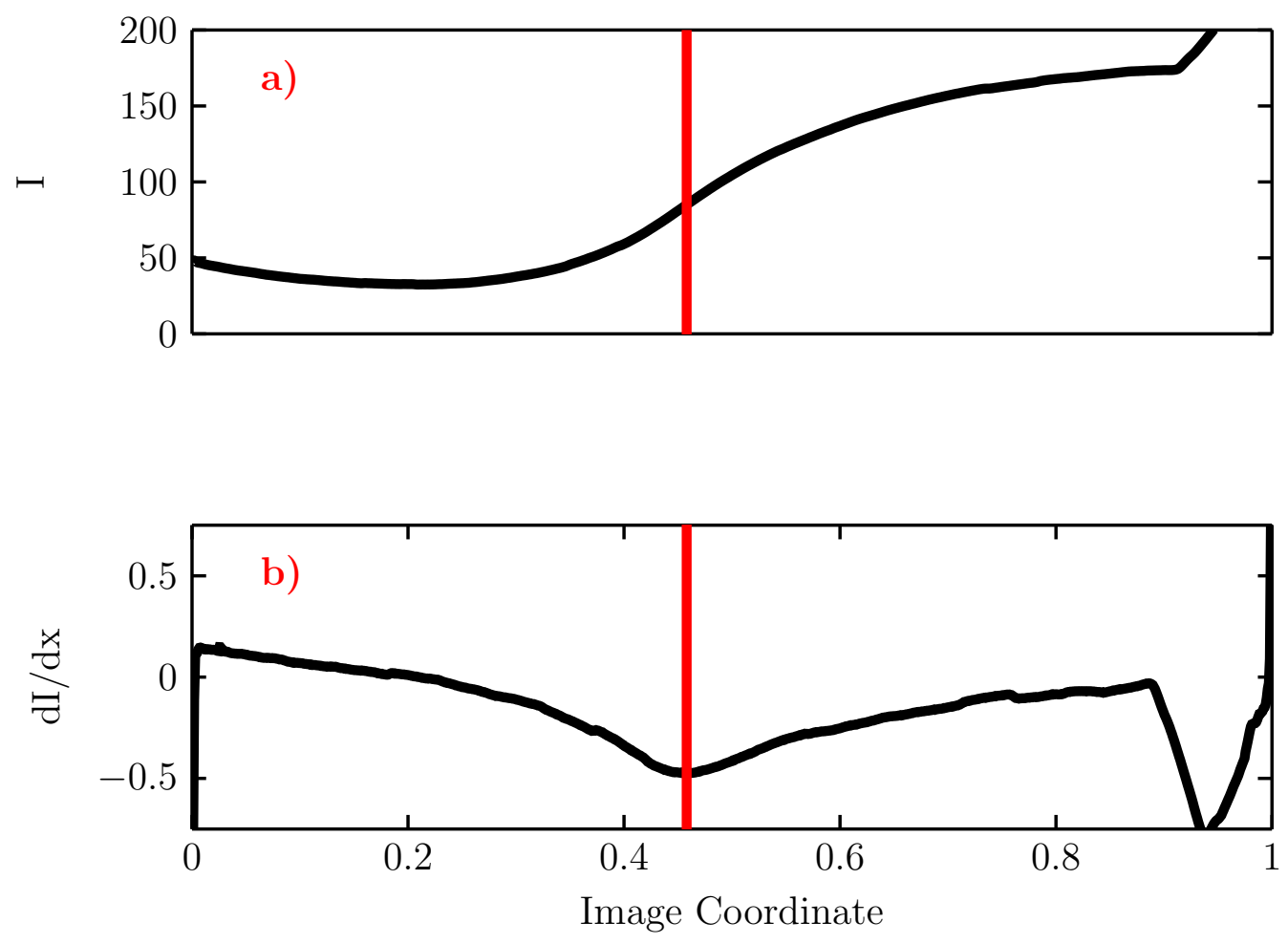

Figure III.11: Example pixel intensity plots. Plots correspond to the NREL S814 upper surface clean configuration at $R e_{c}=3.2 \times 10^{6}$ and an angle of attack of $-9^{\circ}$.

to $x / c$ locations using the airfoil coordinates. Splines are then performed using the image locations of transition and the fiducials as well as the corresponding $x / c$ fiducial locations. These generate the $x / c$ transition locations.

The critical roughness Reynolds number is given by $R e_{k, c r i t}=u_{k} k / \nu_{k}$, where $R e_{k, \text { crit }}$ is the critical roughness Reynolds number, $u_{k}$ is the velocity of the undisturbed boundary layer at the roughness height, $k$ is the roughness height, and $\nu_{k}$ is the kinematic viscosity. To determine $R e_{k, \text { crit }}$ for each roughness configuration of the NACA $63_{3}-418$, the model was held at a constant angle of attack and tunnel velocity was increased until bypass transition occurred. Boundary layer profiles computed at UC Davis were then used to calculate roughness Reynolds numbers along the airfoil 


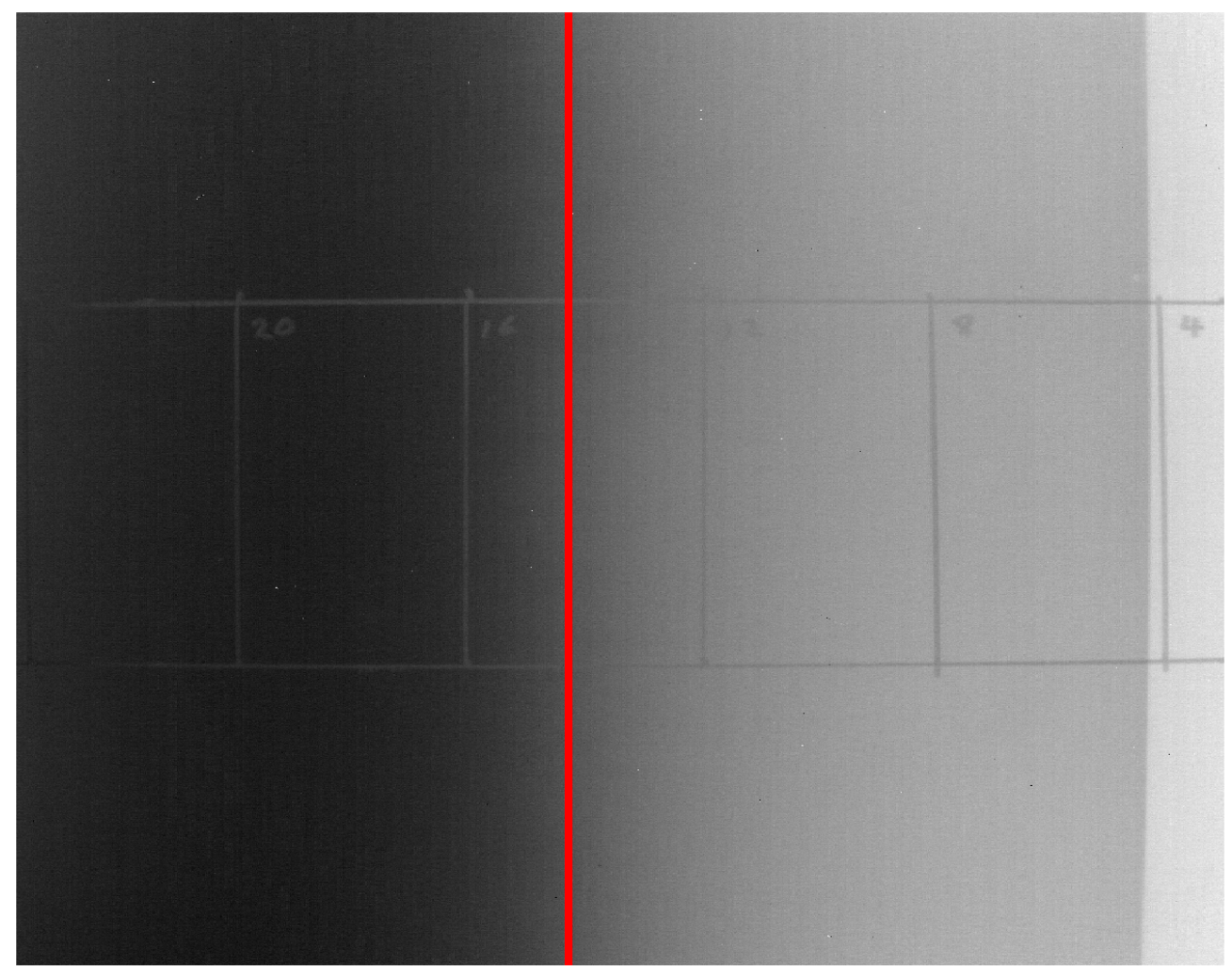

Figure III.12: Example transition detection algorithm output image. Output image corresponds to the NREL S814 upper surface clean configuration at $R e_{c}=3.2 \times 10^{6}$ and an angle of attack of $-9^{\circ}$.

surface. The stagnation point divides flow along the upper and lower surfaces and is characterized by a roughness Reynolds number of zero. The maximum roughness Reynolds number which occurs after the stagnation point and inside the experimental roughness region at the bypass angle of attack was taken as the critical roughness Reynolds number. The process for the NREL S814 data was very similar except that the bypass angle of attack for a constant Reynolds number was used rather than the bypass Reynolds number at a constant angle of attack. This change eliminated the need for extra wind tunnel runs since the bypass angles of attack were already col- 
lected for constant Reynolds numbers during the main testing procedure. Ehrmann found that the value of $R e_{k, c r i t}$ did not vary significantly with angle of attack for the NACA $63_{3}-418$, so it is expected that the change in procedure between these two tests will not significantly affect the results [7]. Figure III.13 shows roughness Reynolds number variations along the NREL S814 airfoil surface corresponding to $100 \mu \mathrm{m}$ roughness height for a variety of angles of attack. The maximum $R e_{k}$ for each angle of attack is marked with a dot, and the roughness limit is marked with a dotted black line. The backward movement of the stagnation point and gradual increase in maximum roughness Reynolds number as angle of attack increases are visible.

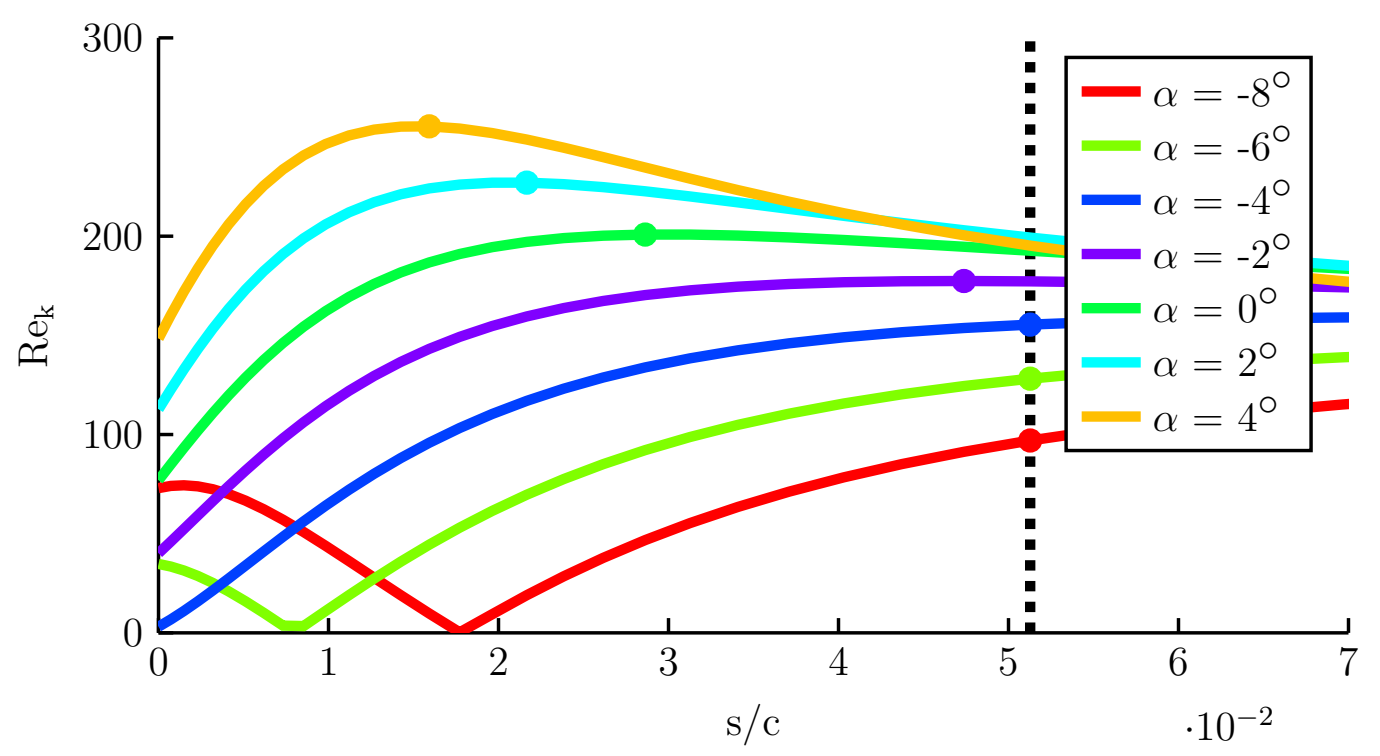

Figure III.13: $R e_{k}$ variation with surface location plot. Plot corresponds to the NREL S814 upper surface at $R e_{c}=3.2 \times 10^{6}$ and $100 \mu \mathrm{m}$ roughness height.

Figure III.14 shows how maximum roughness Reynolds varies with angle of attack on the NREL S814 upper surface at $R e_{c}=3.2 \times 10^{6}$ for a roughness height of 
$100 \mu \mathrm{m}$. The upper and lower bounds of Tani's critical roughness Reynolds number criterion are plotted as dashed lines. The maximum roughness Reynolds number increases with angle of attack due primarily to the movement of the stagnation point onto the lower surface. This exposes a larger region of the upper surface flow to the roughness. The maximum roughness Reynolds number first crosses the lower bound of Tani's correlation near $\alpha=-3^{\circ}$, and exceeds the upper bound near $\alpha=4^{\circ}$. It is expected that bypass transition will occur between these values. The critical roughness Reynolds number can be found by taking $R e_{k, \max }$ from this figure at the bypass angle of attack.

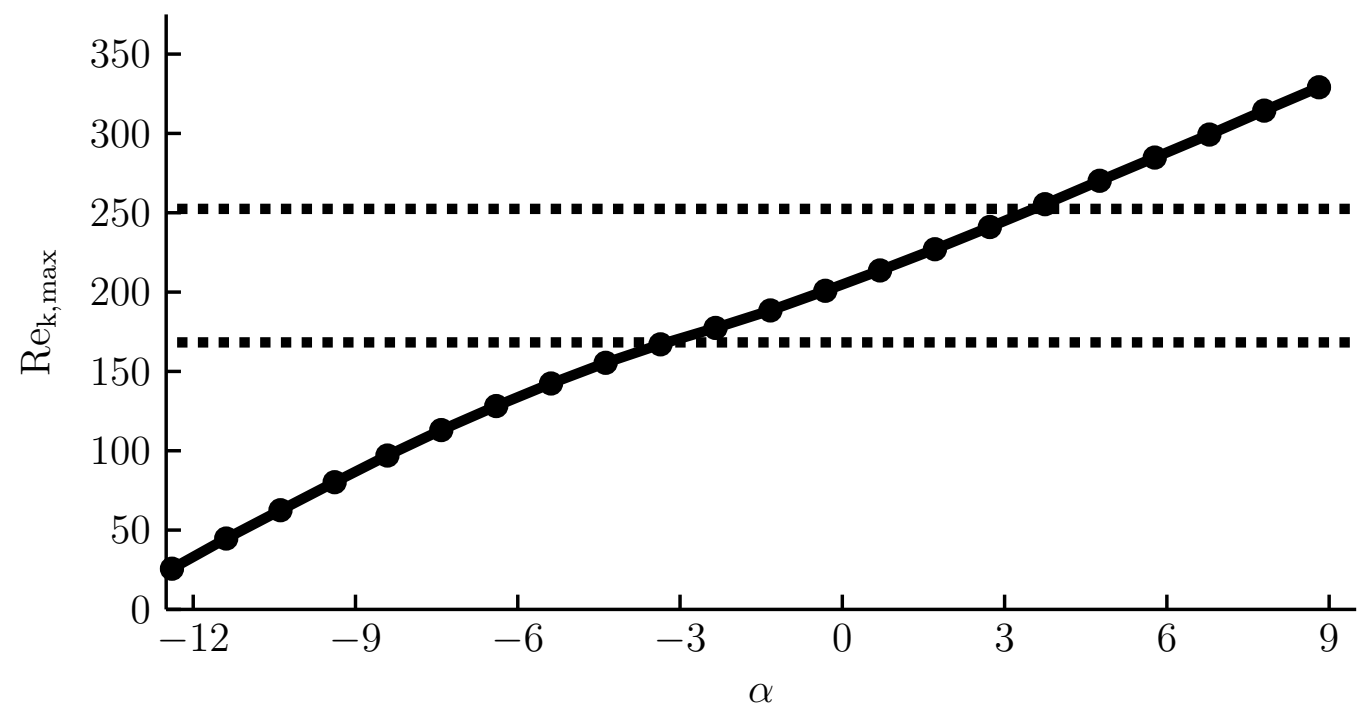

Figure III.14: $R e_{k, \max }$ variation with angle of attack plot. Plot corresponds to the NREL S814 upper surface at $R e_{c}=3.2 \times 10^{6}$ and $100 \mu \mathrm{m}$ roughness height. 


\section{III.I. Lift, Drag, and Pitching Moment Results}

Lift, drag, and pitching moment results are presented here for the NACA $63_{3}-418$ and NREL S814 airfoils. The NACA $63_{3}-418$ results were generated by Ehrmann and are used here as a comparison [7]. Efforts were made to replicate Ehrmann's test methods so that results are directly comparable. All results are presented for $R e_{c}=3.2 \times 10^{6}$. Results for the NREL S814 at other Reynolds numbers are found in Appendices D and E.

Figures III.15-III.17 show lift, pitching moment, and drag variation on the NACA $63_{3}-418$ for each roughness configuration at a Reynolds number of $3.2 \times 10^{6}$. Experimental data from Abbott \& von Doenhoff and results computed with XFOIL using an N-factor of 5 are included for comparison [12] [24]. Error bars are omitted on the lift and pitching moment plots as the error for these quantities was too small to be visible on the plots. Errors for these values are discussed later in this section for the NREL S814 airfoil.

In general, increasing roughness height and roughness density causes the lift curve slope and maximum lift to decrease. The largest decreases occur due to the trip strip which leads to reductions in lift curve slope and $c_{l, \max }$ of $6.2 \%$ and $11.0 \%$, respectively. The trip strip and clean configurations represent bounding cases for the other roughness cases. The largest decreases in lift curve slope and maximum lift for the distributed roughness cases occur for the $100 \mu \mathrm{m} 15 \%$ configuration.

The pitching moment coefficient increases as roughness height and density are increased. A likely explanation is that roughness causes an increase in boundary layer growth which leads to viscous decambering of the airfoil near the trailing edge. 


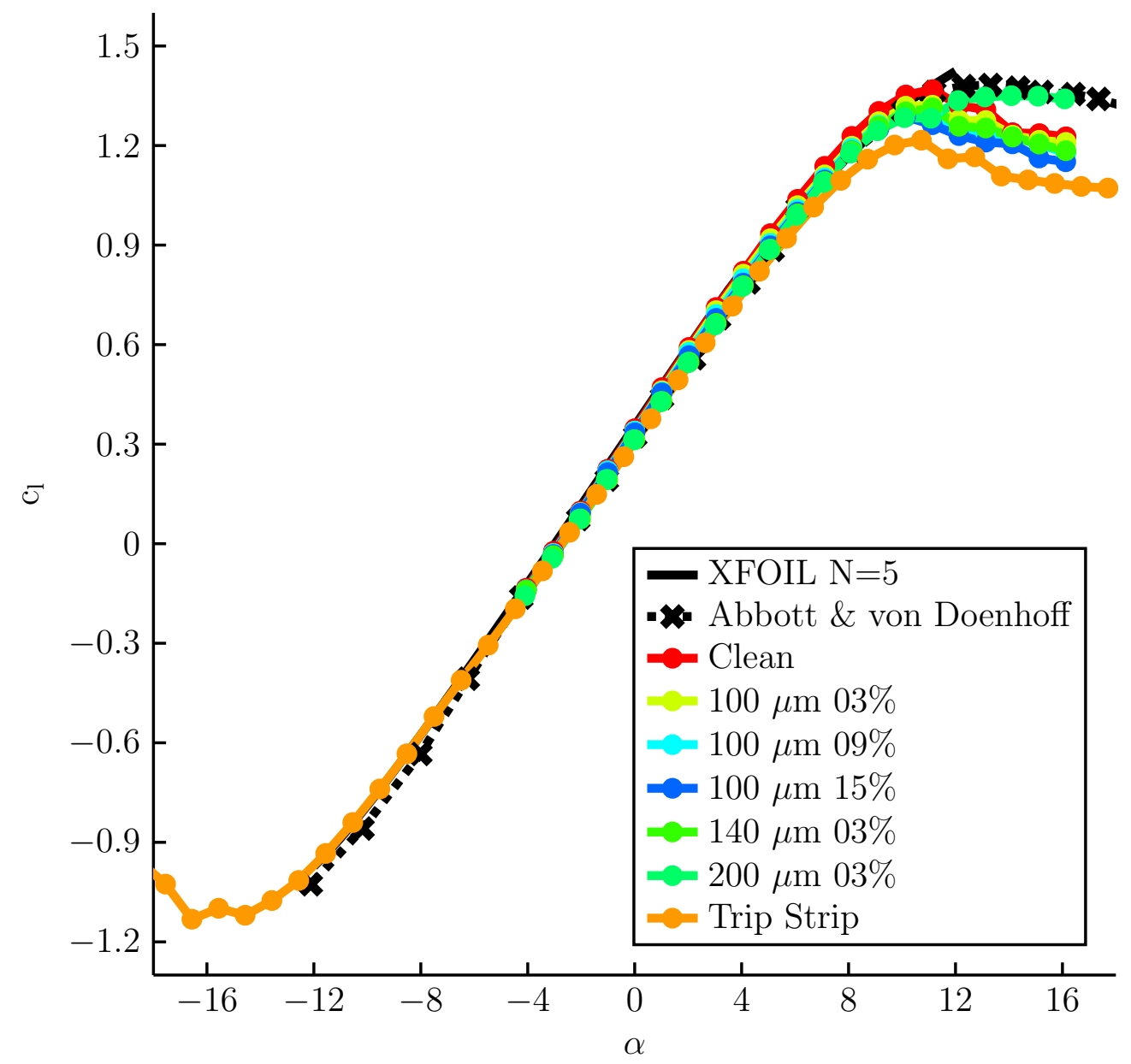

Figure III.15: Lift variation with angle of attack for varying roughness height and density on the NACA $63_{3}-418$ at $R e_{c}=3.2 \times 10^{6}$. 


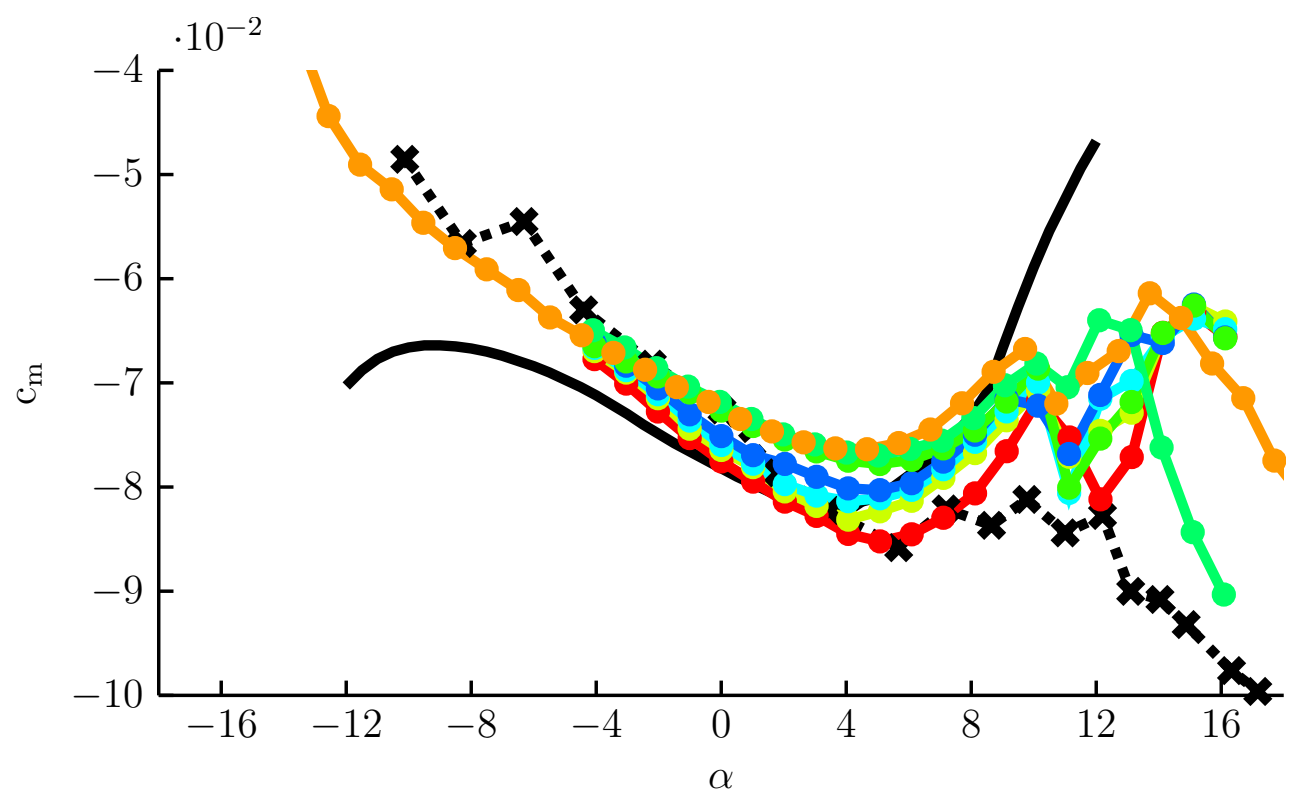

Figure III.16: Pitching moment variation with angle of attack for varying roughness height and density on the NACA $63_{3}-418$ at $R e_{c}=3.2 \times 10^{6}$.

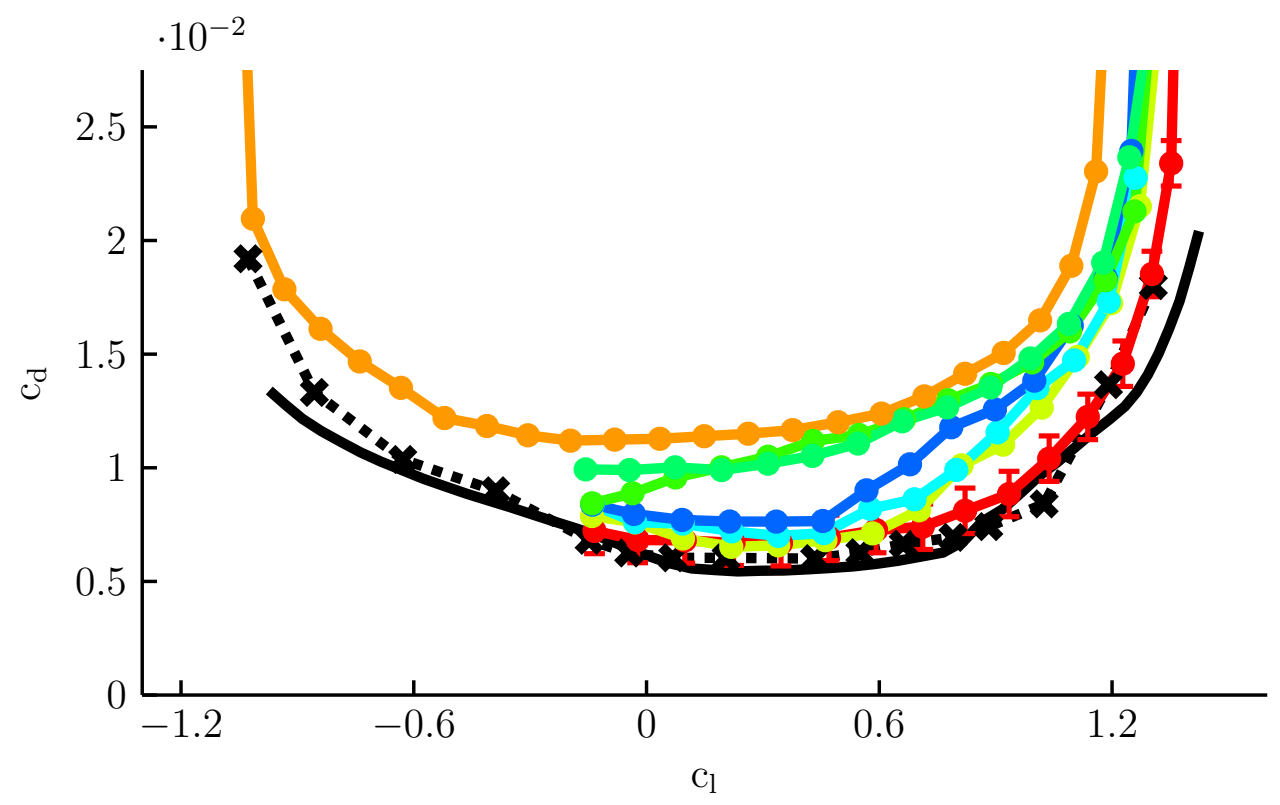

Figure III.17: Drag variation with angle of attack for varying roughness height and density on the NACA $63_{3}-418$ at $R e_{c}=3.2 \times 10^{6}$. 
At an angle of attack around $6^{\circ}$ the pitching moment begins to increase for all configurations and diverges from the Abbott \& von Doenhoff data. The increase in pitching moment is also present in the XFOIL simulation data, so the reason for the discrepancy between the Abbott \& von Doenhoff data was not investigated.

Differences between the various roughness configurations are most evident by comparing the drag polars. Drag coefficients for the distributed roughness configurations fall between the values for the clean and tripped configurations. For low angles of attack, distributed roughness cases converge near the clean values. As angle of attack increases, drag coefficients diverge from the clean values and toward the trip strip values. The $200 \mu \mathrm{m}$ divergence point occurs first and has already occurred for the angles of attack tested. The $140 \mu \mathrm{m}$ configuration diverges next near the lowest lift coefficient tested, followed by the $100 \mu \mathrm{m}$ configurations in order of decreasing density near a lift coefficient of 0.5 . The divergence points correspond to the angles of attack where bypass transition occurs.

Performance losses for the NACA $63_{3}-418 R e_{c}=3.2 \times 10^{6}$ are summarized in Table III.1. All loses are reported as percent differences from the clean configuration values. The clean configuration row contains raw values rather than percent differences. Roughness density had the largest effect on maximum lift and lift curve slope, but roughness height had a larger effect on maximum lift-to-drag ratio. The maximum change in lift-to-drag ratio occurs for the $200 \mu \mathrm{m}$ configuration $(36.6 \%)$.

Figures III.18-III.20 show lift, pitching moment, and drag data for the NREL S814 airfoil at $R e_{c}=3.2 \times 10^{6}$. Experimental data from Somers taken at a Reynolds number of $1.5 \times 10^{6}$ as well as XFOIL data is included in the plots for comparison [22] [24]. 
Table III.1: Performance losses on the NACA $63_{3}-418$ at $R e_{c}=3.2 \times 10^{6}$.

\begin{tabular}{cccc}
\hline \hline Configuration & $d c_{l} / d \alpha$ & $c_{l, \max }$ & $\left(c_{l} / c_{d}\right)_{\max }$ \\
\hline Clean & $6.68 \mathrm{rad}^{-1}$ & 1.37 & 105.66 \\
$100 \mu \mathrm{m} \mathrm{03 \%}$ & $-1.1 \%$ & $-3.2 \%$ & $-17.8 \%$ \\
$100 \mu \mathrm{m} \mathrm{09 \%}$ & $-3.2 \%$ & $-4.4 \%$ & $-23.0 \%$ \\
$100 \mu \mathrm{m} \mathrm{15 \%}$ & $-4.0 \%$ & $-6.2 \%$ & $-31.1 \%$ \\
$140 \mu \mathrm{m} \mathrm{03 \%}$ & $-2.2 \%$ & $-3.9 \%$ & $-35.0 \%$ \\
$200 \mu \mathrm{m} \mathrm{03 \%}$ & $-2.7 \%$ & $-1.5 \%$ & $-36.6 \%$ \\
Trip Strip & $-5.9 \%$ & $-11.0 \%$ & $-41.2 \%$ \\
\hline \hline
\end{tabular}

Increasing roughness height and density caused reductions in lift curve slope and maximum lift. The $100 \mu \mathrm{m} \mathrm{15 \%}$ configuration had the largest decrease in lift curve slope of $11.1 \%$, exceeding even that of the tripped configuration (9.7\%). The trip strip and $200 \mu \mathrm{m}$ configurations showed the largest decreases in $c_{l, \max }$ with $16.2 \%$ and $6.5 \%$ reductions, respectively. At low angles of attack, the roughness caused severe premature stall on the lower surface. This is evident around an angle of attack of $-5^{\circ}$. This stall occurs at higher angles of attack and is more severe for larger roughness heights and densities. Somers found that the main separation mechanism on the NREL S814 lower surface is a laminar-separation bubble [22]. Early bypass transition triggered by roughness results in a new separation mechanism which is likely responsible for the premature stall.

Early lower-surface separation is clearly visible on the pitching moment coefficient plots as a large increase in moment coefficient for low angles of attack. The large magnitude of this increase is due to the airfoil's "S" shape. Lower surface stall is caused by the destruction of the separation bubble on the lower surface near the pressure minimum. The airfoil is very thick at this location, so separation here 


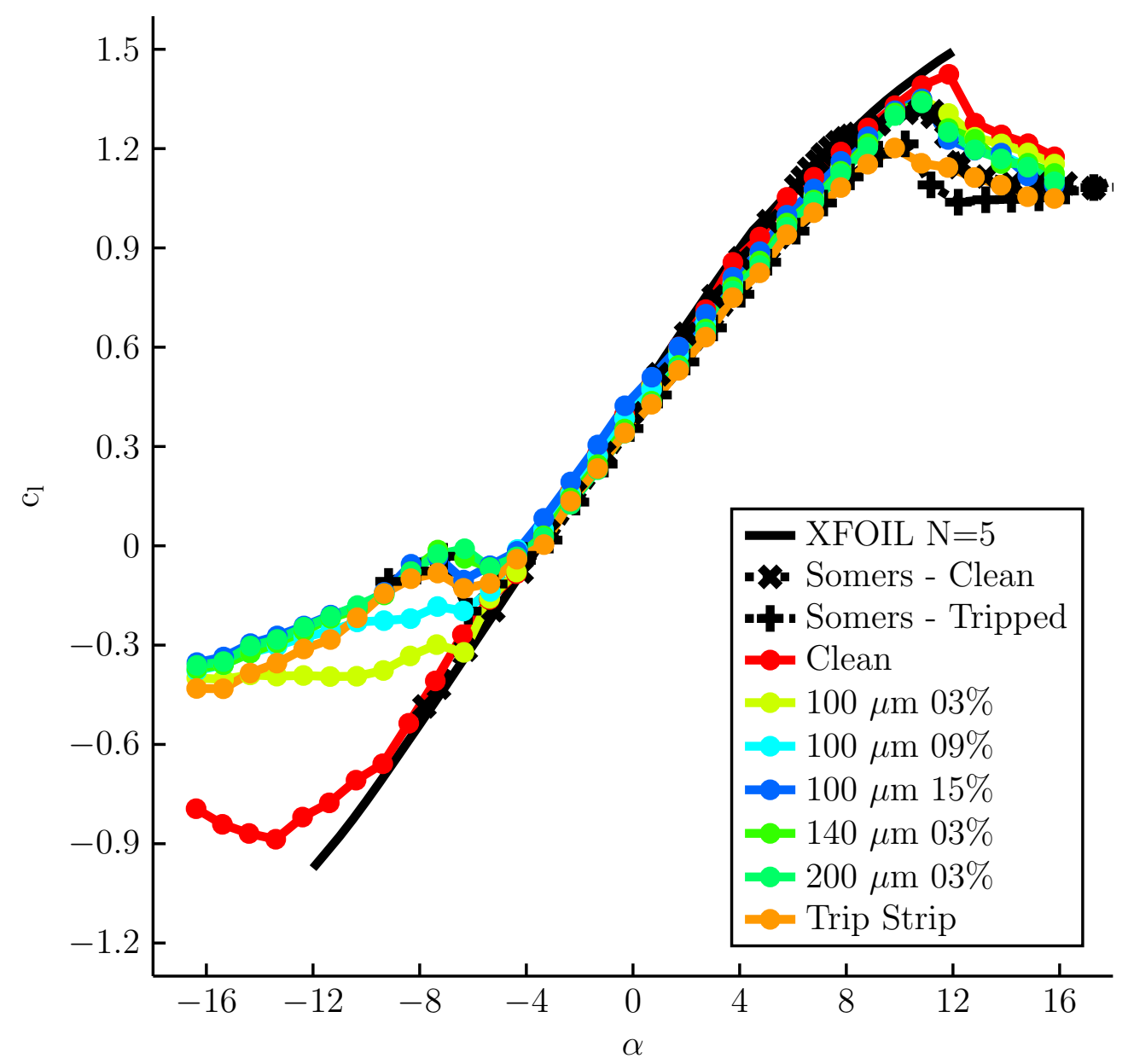

Figure III.18: Lift variation with angle of attack for varying roughness height and density on the NREL S814 at $R e_{c}=3.2 \times 10^{6}$. 


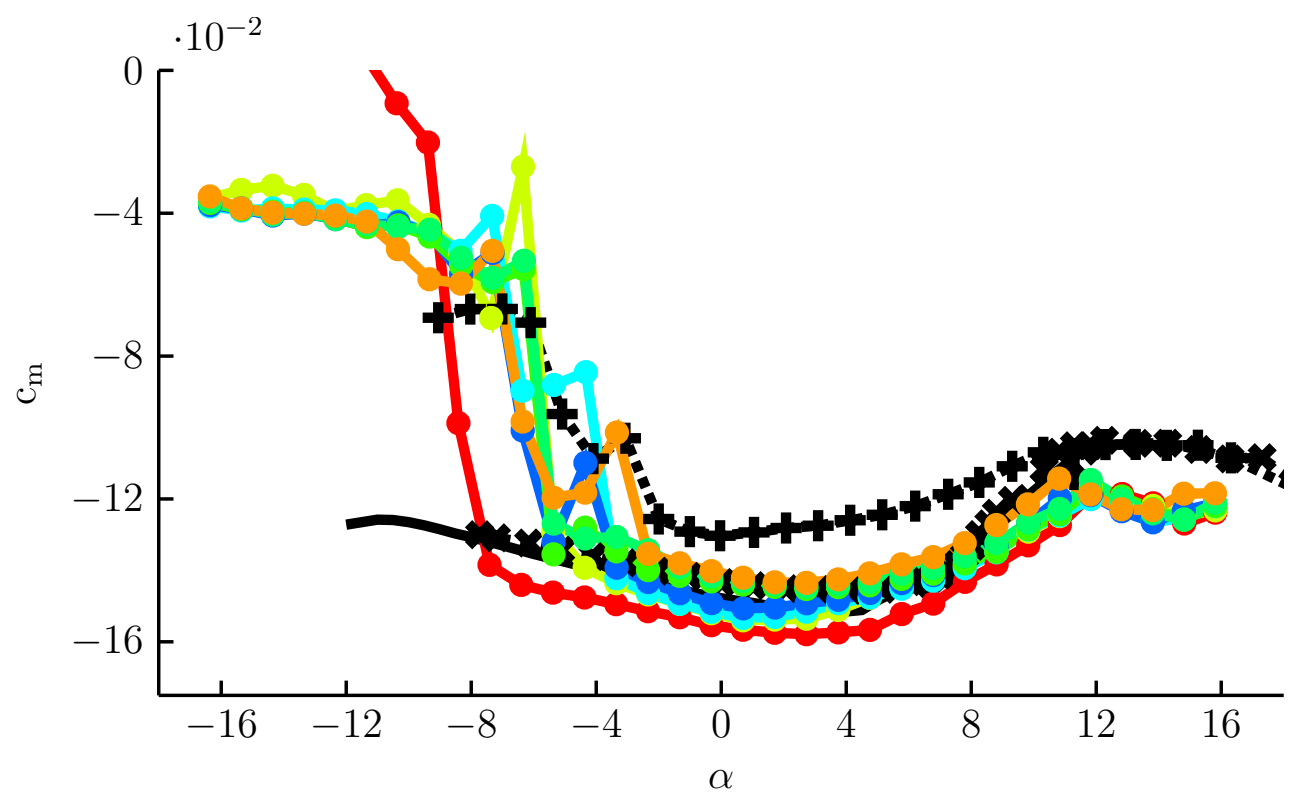

Figure III.19: Pitching moment variation with angle of attack for varying roughness height and density on the NREL S814 at $R e_{c}=3.2 \times 10^{6}$.

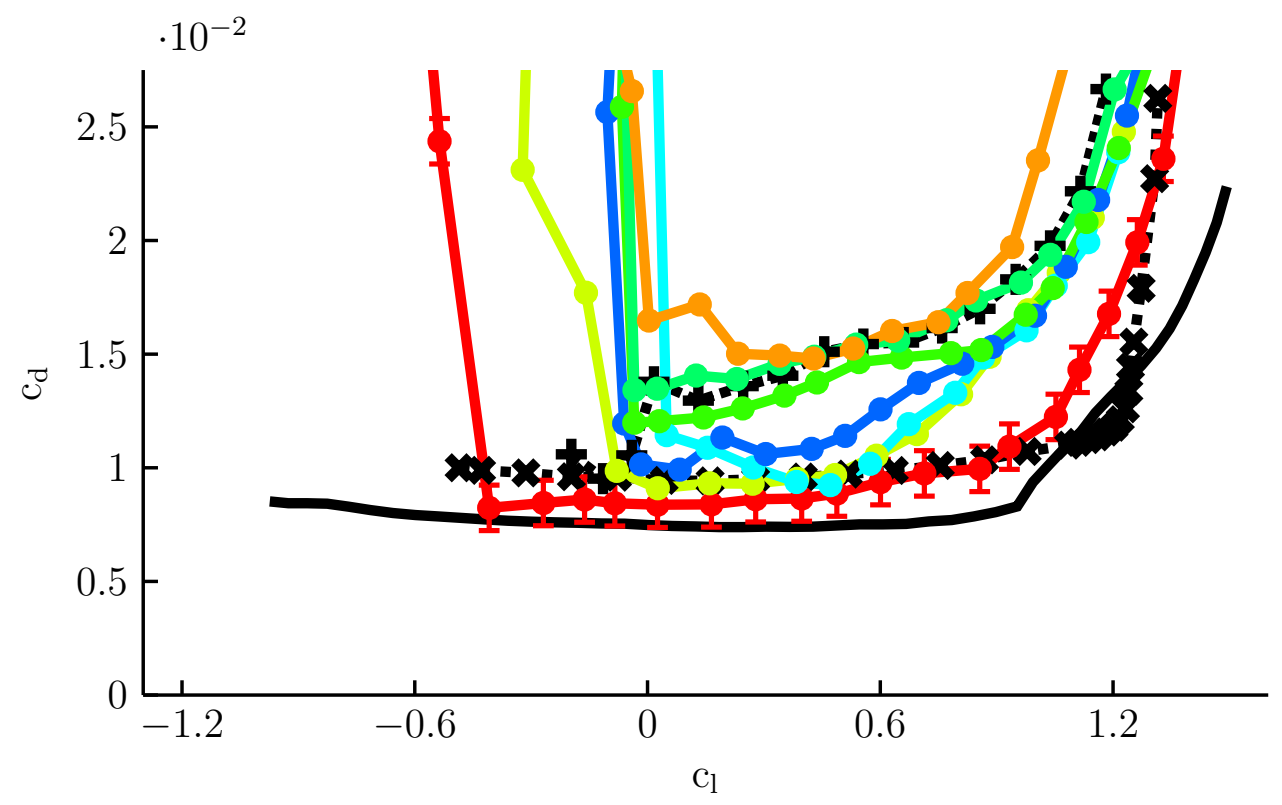

Figure III.20: Drag variation with angle of attack for varying roughness height and density on the NREL S814 at $R e_{c}=3.2 \times 10^{6}$. 


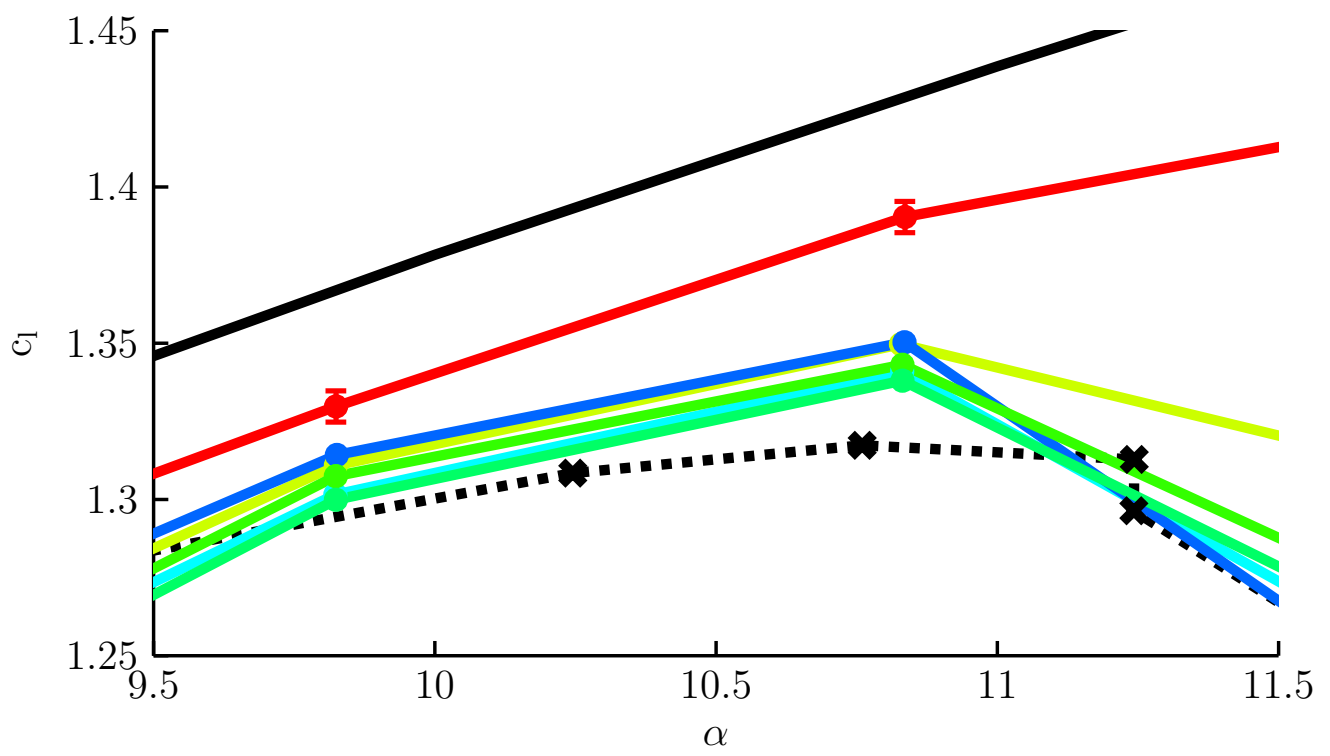

Figure III.21: Lift variation with angle of attack (zoomed version) for varying roughness height and density on the NREL S814 at $R e_{c}=3.2 \times 10^{6}$.

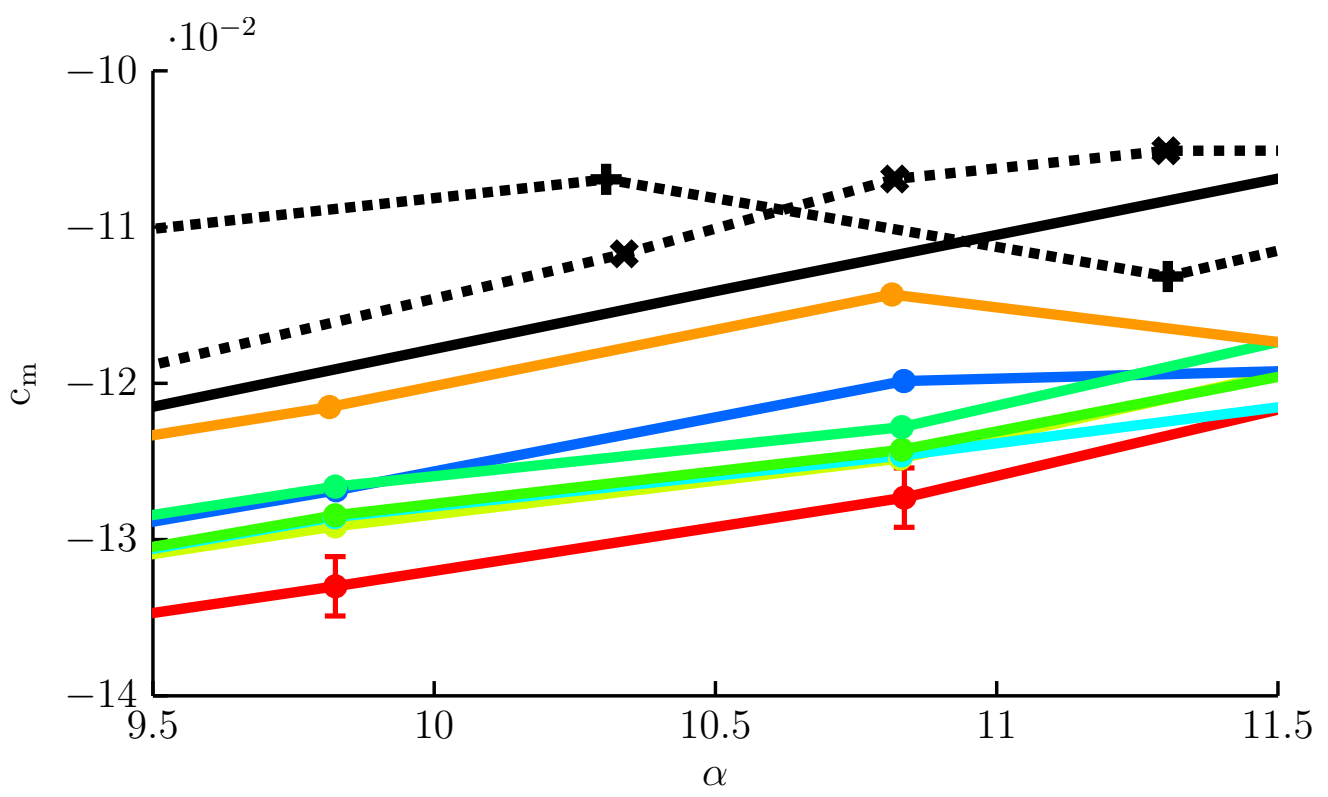

Figure III.22: Pitching moment variation with angle of attack (zoomed version) for varying roughness height and density on the NREL S814 at $R e_{c}=3.2 \times 10^{6}$. 
causes massive decambering of the airfoil. A more physical explanation is that the separated flow exerts a lower pressure on the back half of the airfoil's lower surface than the corresponding attached flow, causing an increase in moment. The pitching moment plots also show that increasing roughness height and density increases the pitching moment even for attached flow. This is consistent with results from the NACA $63_{3}-418$. A slight offset exists between this data and Somers experimental data. The reason for this offset is not clear.

The NREL S814 drag polar provides similar results as that of the NACA $63_{3}-418$, with increasing roughness height and density corresponding to increased drag. Somers data shows a much sharper knee in the drag polar near the stall lift coefficient of approximately 1.2. Differences in tunnel flow quality are likely responsible for this behavior. Somers data also shows a decrease in drag near the lower-surface stall that is markedly different from the more-typical drag rise observed in this experiment.

Figures III.21 and III.22 are zoomed-in lift and pitching moment coefficient plots for the NREL S814 in which the error bars for these quantities are visible. The uncertainties vary slightly with angle of attack but are approximately 0.005 for lift coefficient and 0.002 for pitching moment coefficient. Uncertainties in pressures were very low, so uncertainties in lift and pitching are correspondingly low.

Table III.2 summarizes the airfoil performance data for the NREL S814 at $R e_{c}=3.2 \times 10^{6}$. Data for $R e_{c}=1.6 \times 10^{6}$ and $R e_{c}=2.4 \times 10^{6}$ is reported in Appendix E. Performance losses for $R e_{c}=4.0 \times 10^{6}$ are not included as the angleof-attack range was too limited to generate this data. Excluding the tripped case, maximum lift-to-drag ratio decreases most severely for the $200 \mu \mathrm{m}$ configuration 
(37.8\%). Roughness height has a more pronounced effect than roughness density on maximum lift-to-drag ratio.

Table III.2: Performance losses on the NREL S814 at $R e_{c}=3.2 \times 10^{6}$.

\begin{tabular}{cccc}
\hline \hline Configuration & $d c_{l} / d \alpha$ & $c_{l, \max }$ & $\left(c_{l} / c_{d}\right)_{\max }$ \\
\hline Clean & $6.26 \mathrm{rad}^{-1}$ & 1.43 & 86.74 \\
$100 \mu \mathrm{m} 03 \%$ & $-7.8 \%$ & $-5.9 \%$ & $-29.3 \%$ \\
$100 \mu \mathrm{m} 09 \%$ & $-9.5 \%$ & $-6.2 \%$ & $-29.8 \%$ \\
$100 \mu \mathrm{m} 15 \%$ & $-11.7 \%$ & $-5.3 \%$ & $-31.0 \%$ \\
$140 \mu \mathrm{m} 03 \%$ & $-5.7 \%$ & $-6.0 \%$ & $-32.4 \%$ \\
$200 \mu \mathrm{m} 03 \%$ & $-5.6 \%$ & $-6.4 \%$ & $-37.8 \%$ \\
Trip Strip & $-9.6 \%$ & $-16.2 \%$ & $-44.9 \%$ \\
\hline \hline
\end{tabular}

Figures III.23 and III.24 demonstrate how lift and drag vary with Reynolds number for the NREL S814 clean configuration. Experimental data from Somers and XFOIL results using an N-factor of 5 are included for comparison [22] [24]. Increasing Reynolds number increases the maximum lift coefficient. As Reynolds number increases, the boundary layer thickness decreases, resulting in a larger stall angle of attack. Interestingly, this trend is reversed on the lower surface, with increasing Reynolds number resulting in decreased magnitude of the lower-surface stall angle of attack. The minimum lift coefficient varies from -1.1 at $R e_{c}=1.6 \times 10^{6}$ to -0.9 at $R e_{c}=3.2 \times 10^{6}$. It is possible that, even for the clean case, small surface imperfections may cause the boundary layer to thicken and prematurely separate for higher Reynolds numbers. The large thickness and correspondingly large pressure gradients on the lower surface makes its separation location especially sensitive to changes in flow and roughness conditions. In general, drag increases slightly with 
increasing Reynolds number, possibly due to the same surface imperfections that result in premature stall.

Reynolds number variations for the $100 \mu \mathrm{m} 03 \%$ roughness configuration are given by Figures III.25 and III.26. Increasing Reynolds number results in a decrease in boundary layer thickness. The relative height of the roughness in comparison to the boundary layer consequently increases so that the effect of the roughness is amplified. The beneficial effects of increased Reynolds number such as increased

maximum lift compete against the detrimental effects of larger roughness. This results in little change at high angles of attack but a severely increased lower-surface stall angle of attack and minimum lift. Increasing Reynolds number decreases the width of the drag bucket and increases the airfoil's minimum drag. The increase in $c_{d, \min }$ is approximately 0.003 between the $R e_{c}=1.6 \times 10^{6}$ and $R e_{c}=4.0 \times 10^{6}$ cases. A change in lower-surface stall lift coefficient of approximately 0.8 between the $R e_{c}=1.6 \times 10^{6}$ and $R e_{c}=3.2 \times 10^{6}$ cases is evident by examining the low-lift portion of the drag bucket, further illustrating the severe sensitivity of the lower surface to changes in flow conditions.

\section{III.J. Transition Location Results}

Transition and critical roughness Reynolds number results are compiled here for both the NACA $63_{3}-418$ airfoil and the NREL S814 airfoil. NACA $63_{3}-418$ results were determined by Ehrmann for the upper surface only. Both upper and lower surface results are given for the NREL S814. Additional results for the NREL S814 are compiled in Appendices F and G. Critical roughness Reynolds number results are 


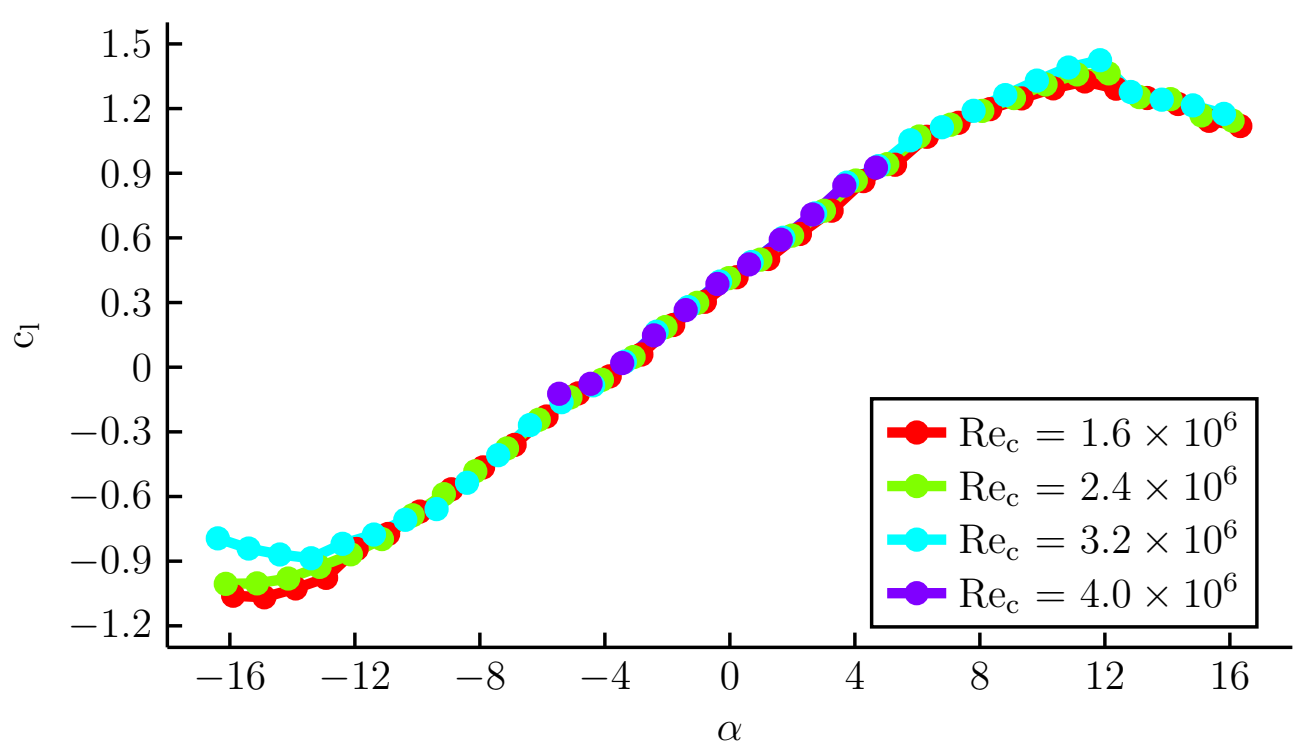

Figure III.23: Lift variation with angle of attack for varying Reynolds number on the NREL S814 clean configuration.

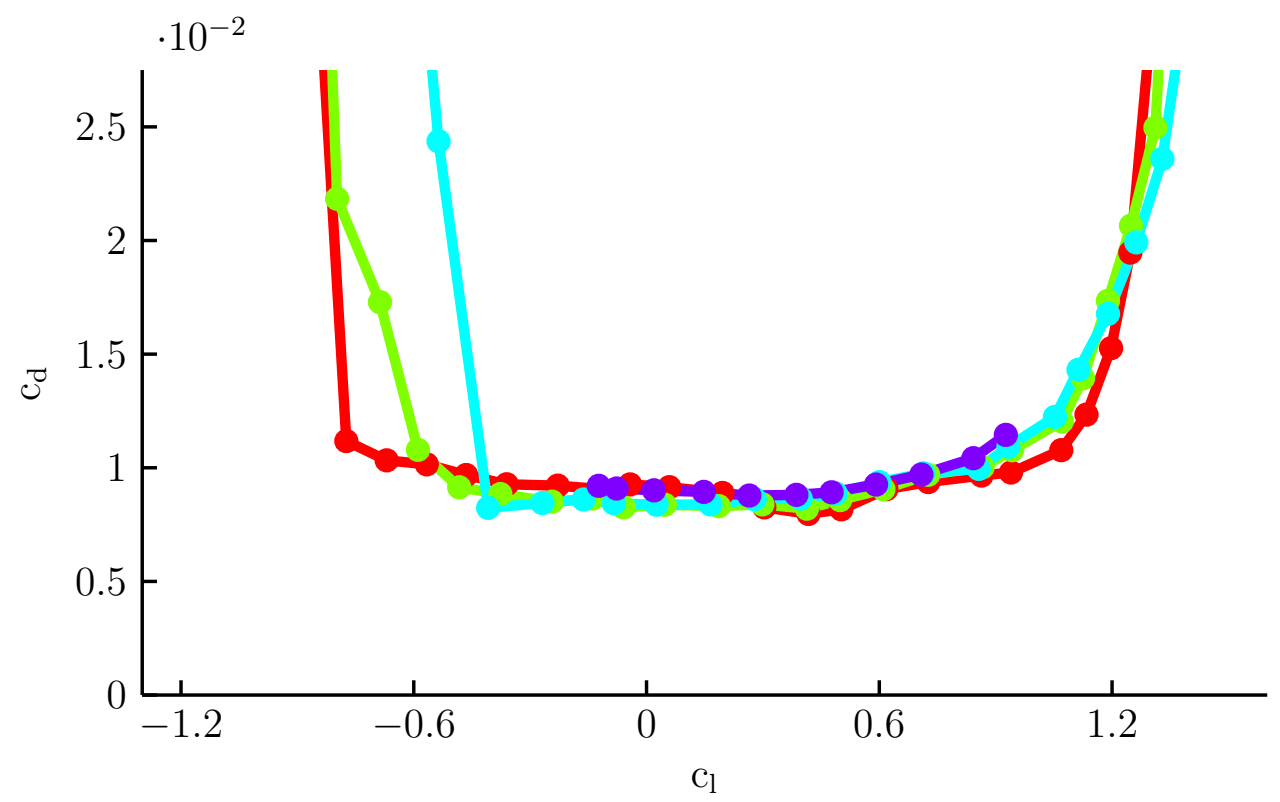

Figure III.24: Drag variation with angle of attack for varying Reynolds number on the NREL S814 clean configuration. 


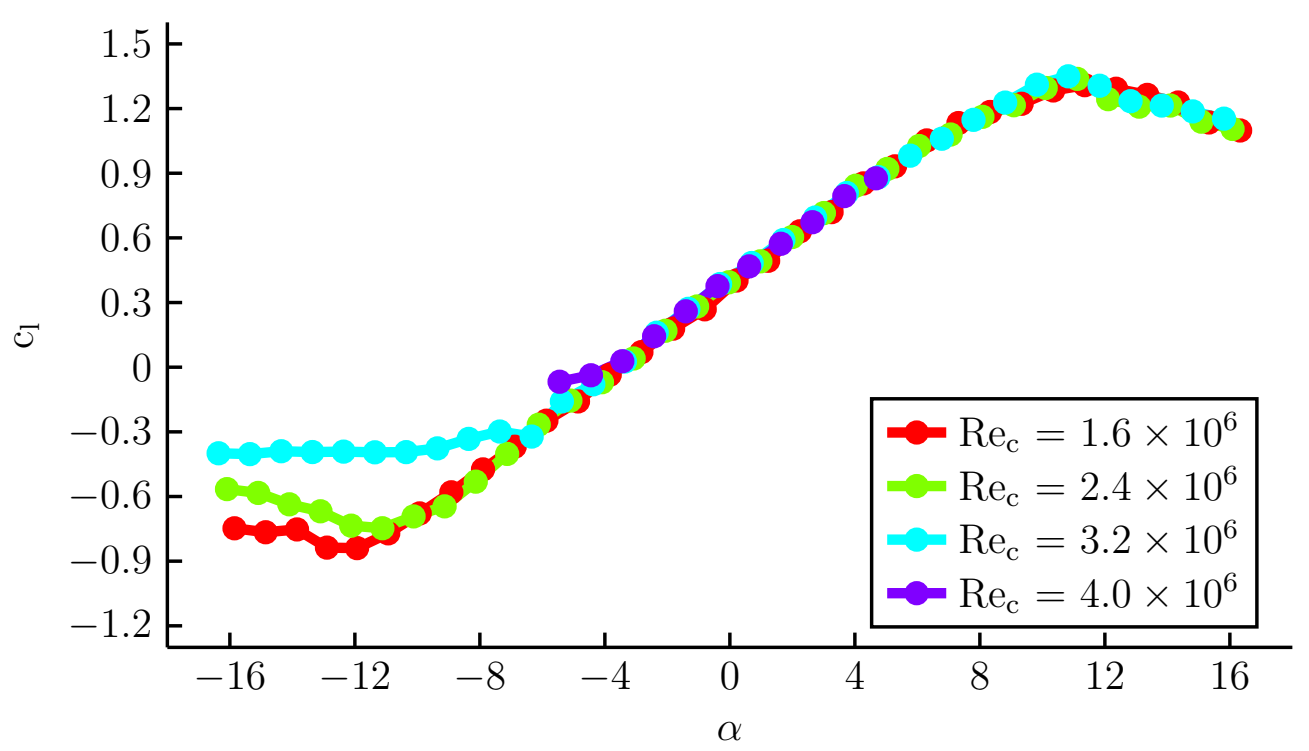

Figure III.25: Lift variation with angle of attack for varying Reynolds number on the NREL S814 $100 \mu \mathrm{m}$ 03\% configuration.

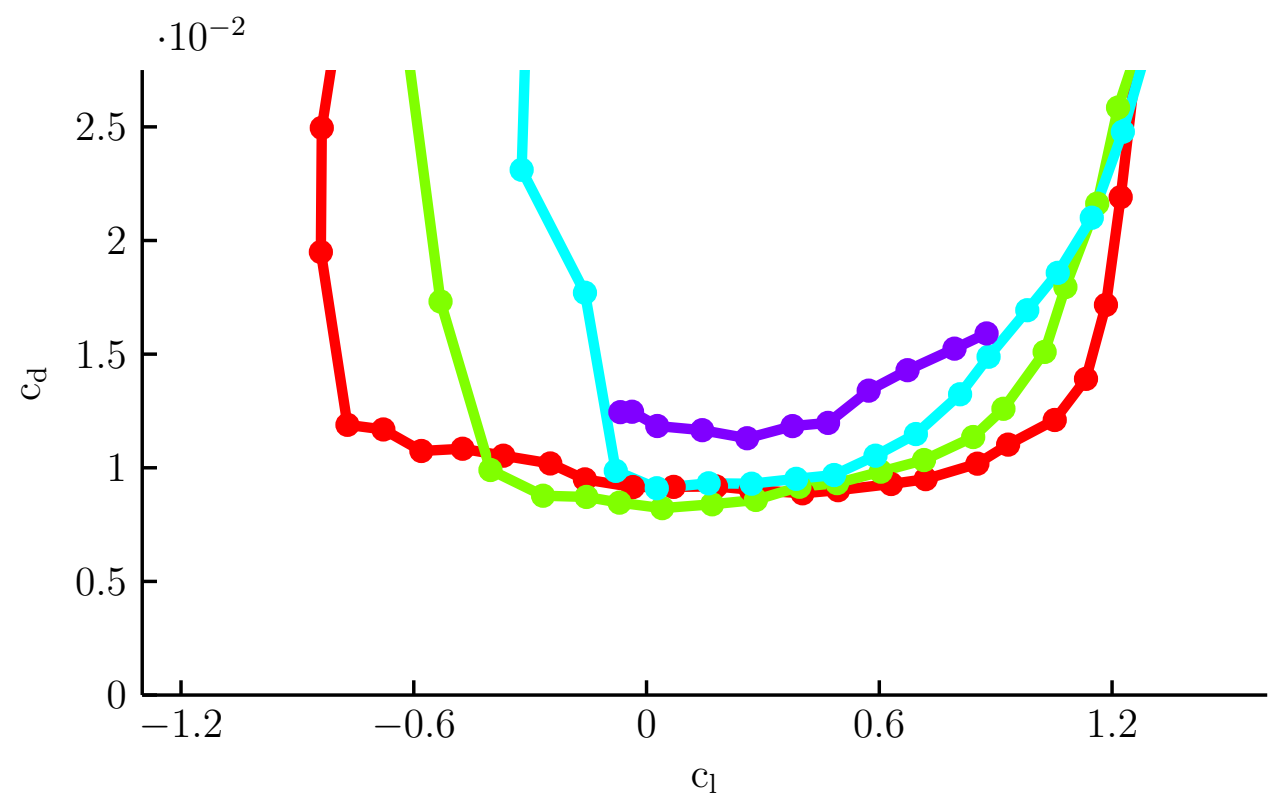

Figure III.26: Drag variation with angle of attack for varying Reynolds number on the NREL S814 $100 \mu \mathrm{m}$ 03\% configuration. 
also presented for each airfoil. Differences in experimental methods for determining critical roughness Reynolds numbers between Ehrmann's test and the current test are discussed briefly.

Transition location results for the upper surface of the NACA $63_{3}-418$ at a Reynolds number of $3.2 \times 10^{6}$ are given in Figure III.27. The bypass angles of attack are denoted by arrows. The minimum pressure locations and transition locations predicted by XFOIL using an N-factor method are included for comparison. The N-factor method is commonly used but must be calibrated for different wind tunnels and tunnel models to account for variation in turbulence intensity values and model surface conditions. Mack provides one of the earliest models which predicts a critical N-factor that varies with the natural log of turbulence intensity, $N_{\text {crit }}=-8.43-2.4 \ln (T u)$. This method predicts a critical N-factor 3.2 for the turbulence intensity found in the LSWT $(T u=0.8 \%)$, although previous tests in this tunnel have revealed that $N_{c r i t}$ of around 5 is appropriate. A critical N-factor of 5 is used here, but additional testing may reveal that a lower value is more appropriate.

Bypass has already occurred at the lowest angle of attack for the $200 \mu \mathrm{m}$ roughness configuration, so this configuration is not included in Figure III.27. As angle of attack increases, bypass begins to occur for the other roughness configurations: first the $140 \mu \mathrm{m}$ configuration followed by the $100 \mu \mathrm{m}$ configurations in order of decreasing density. Bypass angles of attack for the different densities of the $100 \mu \mathrm{m}$ cases differ by less than $2^{\circ}$, but bypass angles of attack between the $100 \mu \mathrm{m} 03 \%$ and $140 \mu \mathrm{m} 03 \%$ cases differ by $6^{\circ}$. This suggest that bypass angles of attack are much more dependent on roughness height than on roughness density. Experimen- 


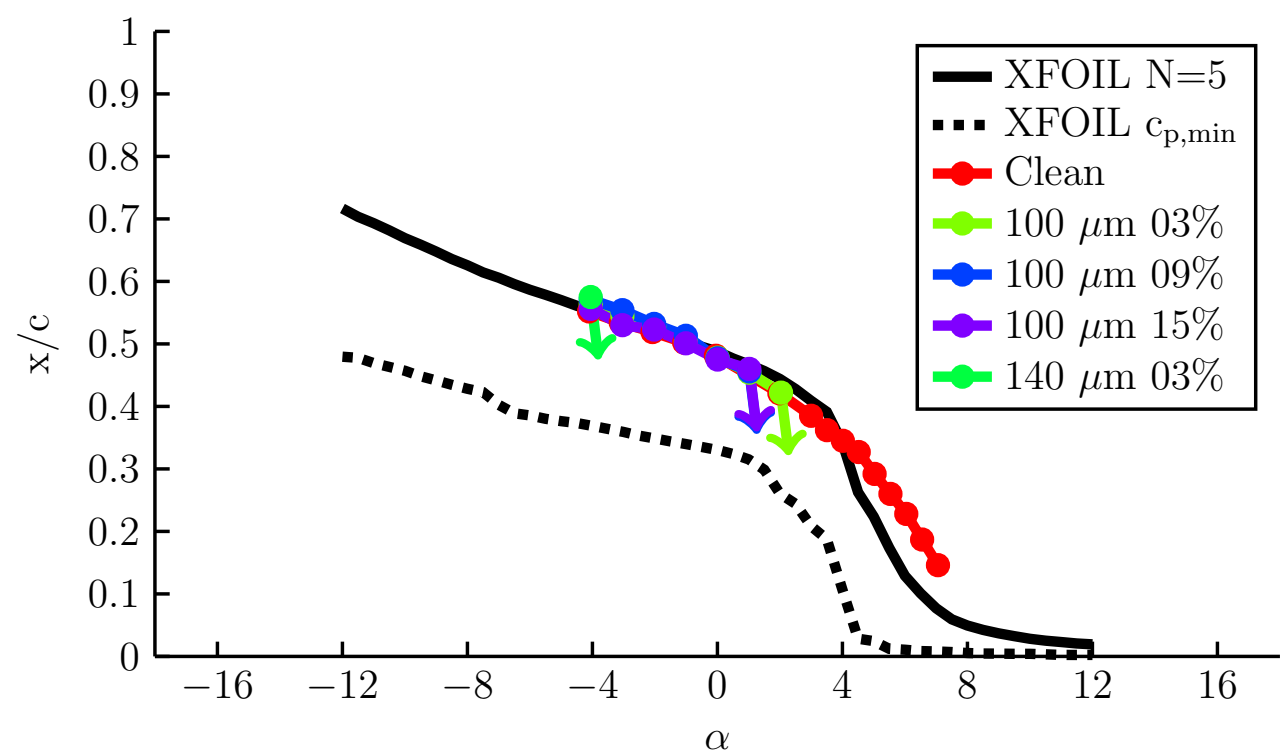

Figure III.27: Upper surface transition location variation with angle of attack for varying roughness height and density on the NACA $63_{3}-418$ at $R e_{c}=3.2 \times 10^{6}$.

tal results show a more gradual movement of transition front at an angle of attack around $4^{\circ}$ than is predicted by XFOIL, but experimental and computational results are otherwise consistent.

Critical roughness Reynolds numbers for each configuration of the NACA $63_{3}-418$ are given in Table III.3. Tani's critical roughness Reynolds number correlation discussed in Section I.D provides a range of expected $R e_{k, c r i t}$ values for a given roughness height. The experimental $R e_{k, c r i t}$ values for the $100 \mu \mathrm{m} 03 \%$ and $100 \mu \mathrm{m} 09 \%$ cases fall outside the range of expected values, but all other cases are within the historical limits. Uncertainties in the experimental critical roughness Reynolds number values are based on the uncertainty in tunnel velocity.

Figures III.28 and III.29 show transition locations and bypass angles of attack on the NREL S814 at $R e_{c}=3.2 \times 10^{6}$. As roughness height and density increases, bypass 
Table III.3: Critical roughness Reynolds numbers on the NACA $63_{3}-418$ upper surface at $R e_{c}=3.2 \times 10^{6}$.

\begin{tabular}{ccc}
\hline \hline Configuration & $R e_{k, \text { crit }}[$ Experimental] & $R e_{k, \text { crit }}$ [Tani Correlation] \\
\hline $100 \mu \mathrm{m} \mathrm{03 \%}$ & $318 \pm 14$ & $168-252$ \\
$100 \mu \mathrm{m} \mathrm{09 \%}$ & $270 \pm 14$ & $168-252$ \\
$100 \mu \mathrm{m} 15 \%$ & $254 \pm 14$ & $168-252$ \\
$140 \mu \mathrm{m} \mathrm{03 \%}$ & $240 \pm 19$ & $193-289$ \\
$200 \mu \mathrm{m} \mathrm{03 \%}$ & $227 \pm 29$ & $239-358$ \\
\hline \hline
\end{tabular}

moves to lower angles of attack on the upper surface and higher angles of attack on the lower surface. Upper surface transition follows the XFOIL $N=5$ curve until an angle of attack of $-6^{\circ}$ where XFOIL predicts transition further downstream on the airfoil. Using lower values of critical N-factor could correct this simulation, but lower values are not consistent with previous experiments in this tunnel. On the lower surface, transition location moves slightly forward as angle of attack is increased. However, this movement is very slight, so the transition location stays near the point of maximum thickness for all angles of attack. The NREL S814 pressure coefficient plot given in Section II.E reveals that pressure gradient changes sharply from highly favorable to highly adverse at $x / c$ near 0.2 . The highly adverse pressure gradient at this location quickly destabilizes the boundary layer and leads to transition.

Critical roughness Reynolds numbers are given for the NREL S814 in Tables III.4 and III.5. Uncertainties here are based on the uncertainty in angle of attack. Bypass had already occurred on the lower surface for all tested angles of attack for the $200 \mu \mathrm{m}$ roughness configuration, so data for this case is not included. Unlike the NACA $63_{3}-418$ cases, these roughness Reynolds numbers were determined at nonzero 


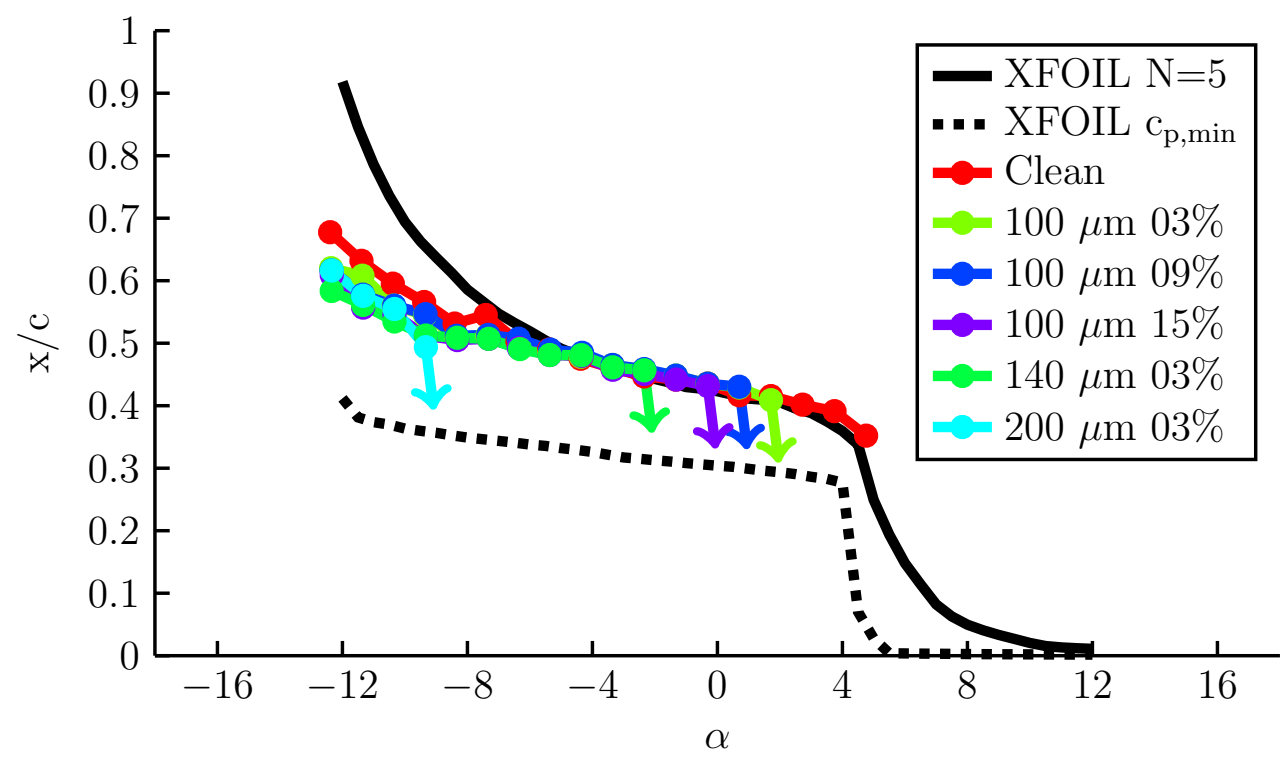

Figure III.28: Upper surface transition location variation with angle of attack for varying roughness height and density on the NREL S814 at $R e_{c}=3.2 \times 10^{6}$.

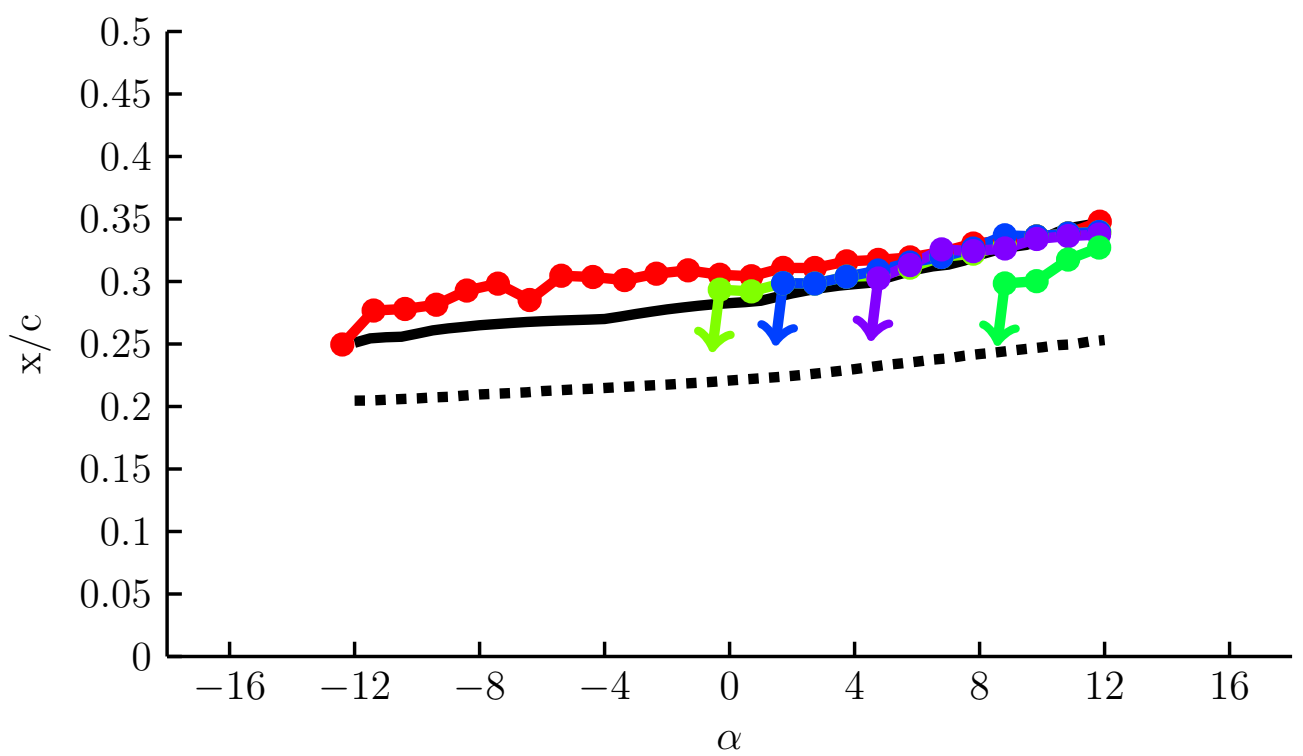

Figure III.29: Lower surface transition location variation with angle of attack for varying roughness height and density on the NREL S814 at $R e_{c}=3.2 \times 10^{6}$. 
angles of attack, so the pressure gradients around the airfoil are likely larger than those seen on the NACA $63_{3}-418$. Smith \& Clutter state that the effect of pressure gradient on critical roughness Reynolds number is minimal [28]. All critical values fall within the range offered by Tani's correlation.

Critical roughness values approach Tani's lower bound for large roughness densities. Furthermore, all the $100 \mu \mathrm{m}$ roughness cases satisfy the correlation despite spanning a bypass angle-of-attack range of $4^{\circ}$. The large range and independence from roughness density of Tani's correlation coefficient may prevent the correlation from being used to predict bypass angles of attack with low uncertainties.

Table III.4: Critical roughness Reynolds numbers on the NREL S814 upper surface at $R e_{c}=3.2 \times 10^{6}$.

\begin{tabular}{ccc}
\hline \hline Configuration & $R e_{k, \text { crit }}$ [Experimental] & $R e_{k, \text { crit }}$ [Tani Correlation] \\
\hline $100 \mu \mathrm{m} \mathrm{03 \%}$ & $227-241$ & $168-252$ \\
$100 \mu \mathrm{m} \mathrm{09 \%}$ & $214-227$ & $168-252$ \\
$100 \mu \mathrm{m} \mathrm{15 \%}$ & $201-214$ & $168-252$ \\
$140 \mu \mathrm{m} \mathrm{03 \%}$ & $296-313$ & $193-289$ \\
$200 \mu \mathrm{m} \mathrm{03 \%}$ & $278-342$ & $239-358$ \\
\hline \hline
\end{tabular}

Table III.5: Critical roughness Reynolds numbers on the NREL S814 lower surface at $R e_{c}=3.2 \times 10^{6}$.

\begin{tabular}{ccc}
\hline \hline Configuration & $R e_{k, \text { crit }}$ [Experimental] & $R e_{k, \text { crit }}$ [Tani Correlation] \\
\hline $100 \mu \mathrm{m} \mathrm{03 \%}$ & $213-219$ & $168-252$ \\
$100 \mu \mathrm{m} \mathrm{09 \%}$ & $199-206$ & $168-252$ \\
$100 \mu \mathrm{m} \mathrm{15 \%}$ & $176-184$ & $168-252$ \\
$140 \mu \mathrm{m} \mathrm{03 \%}$ & $237-252$ & $193-289$ \\
$200 \mu \mathrm{m} \mathrm{03 \%}$ & - & $239-358$ \\
\hline \hline
\end{tabular}


The effect of Reynolds number on transition location for the NREL S814 clean configuration is shown in Figures III.30 and III.31. Increasing Reynolds number causes the transition location to move forward on both surfaces, but the effects are more pronounced on the upper surface, particularly for low angles of attack. Again, this is due to the highly adverse pressure gradient near the lower surface's point of maximum thickness which fixes its transition location immediately downstream of this point.

Figures III.32 and III.33 demonstrate how Reynolds number changes the transition behavior for the $100 \mu \mathrm{m} 03 \%$ roughnesss configuration. As noted in Section III.F, increasing Reynolds number decreases the size of the boundary layer so that the relative size of the roughness is increased. This increases the effect of the roughness and results in earlier boundary layer transition. As Reynolds number increases, bypass occurs at lower angles of attack on the upper surface and higher angles of attack on the lower surface. Between $R e_{c}=2.4 \times 10^{6}$ and $R e_{c}=3.2 \times 10^{6}$, the bypass angle of attack shifts $3^{\circ}$, so the effect is substantial. 


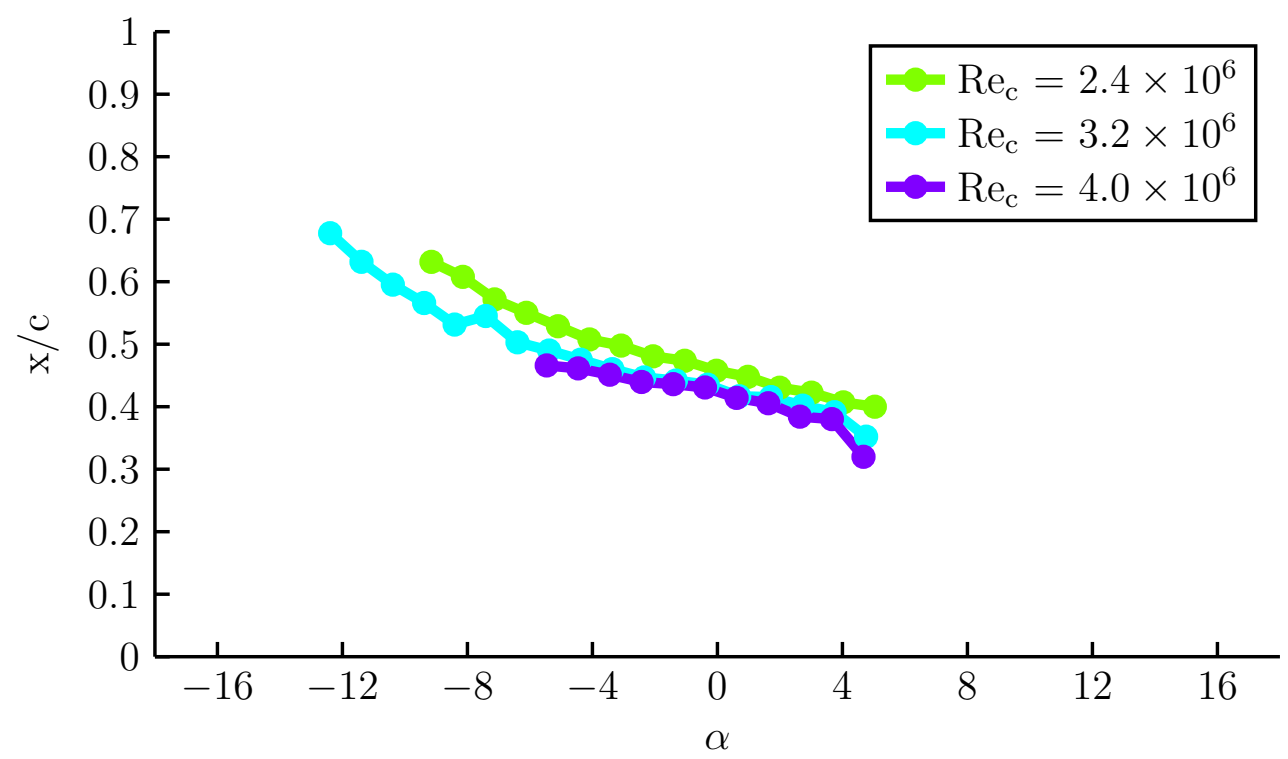

Figure III.30: Upper surface transition location variation with angle of attack for varying Reynolds number on the NREL S814 clean configuration.

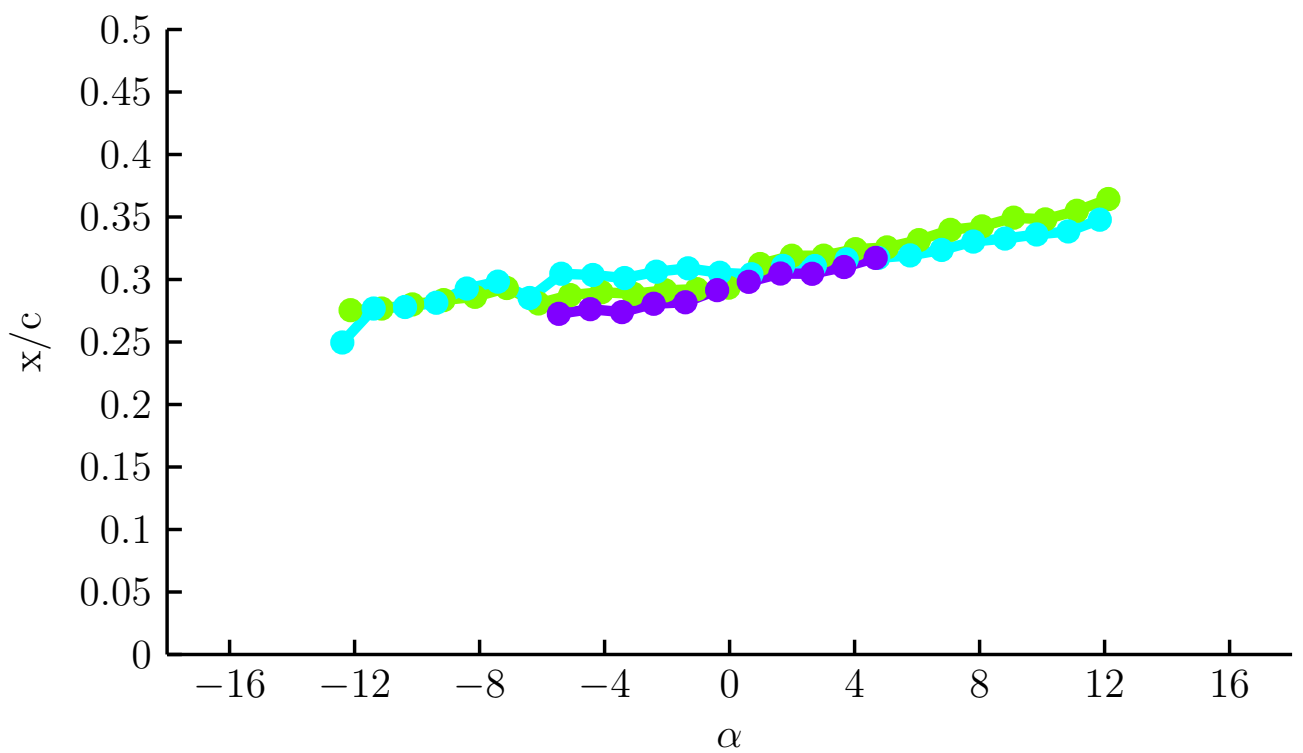

Figure III.31: Lower surface transition location variation with angle of attack for varying Reynolds number on the NREL S814 clean configuration. 


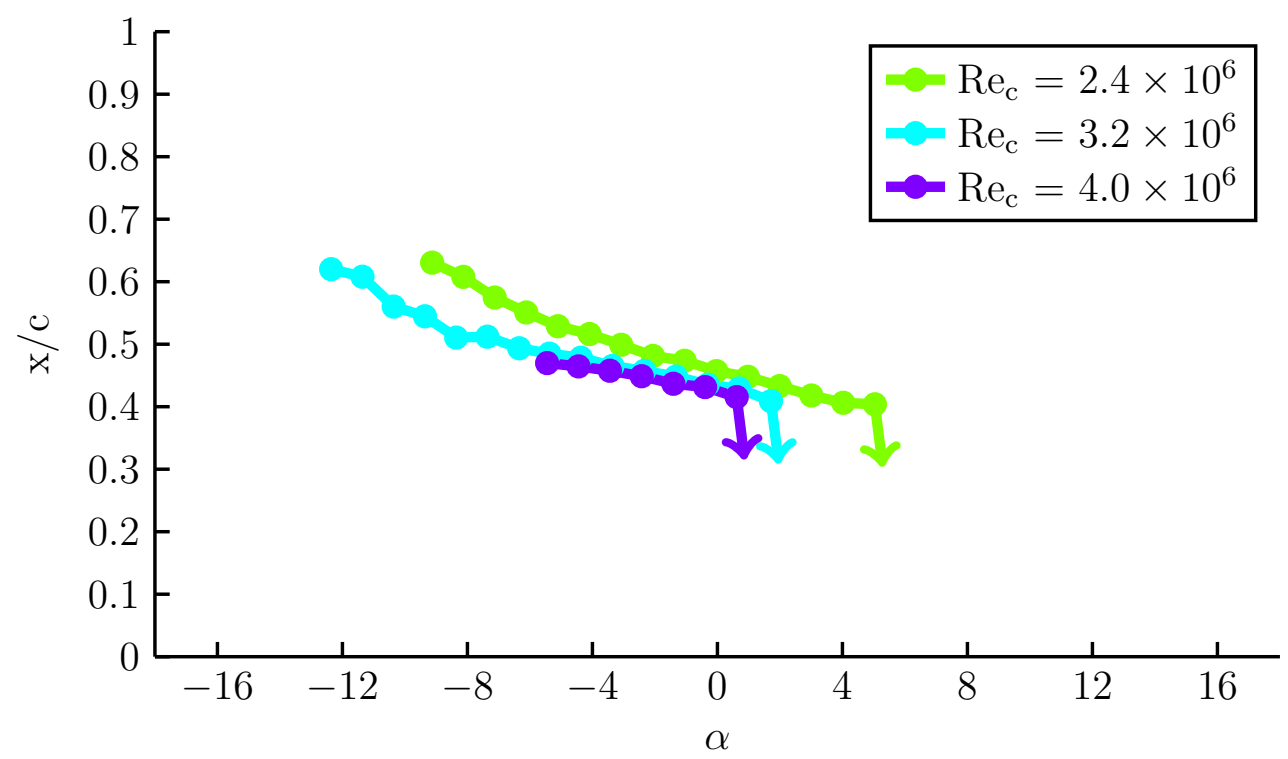

Figure III.32: Upper surface transition location variation with angle of attack for varying Reynolds number on the NREL S814 $100 \mu \mathrm{m}$ 03\% configuration.

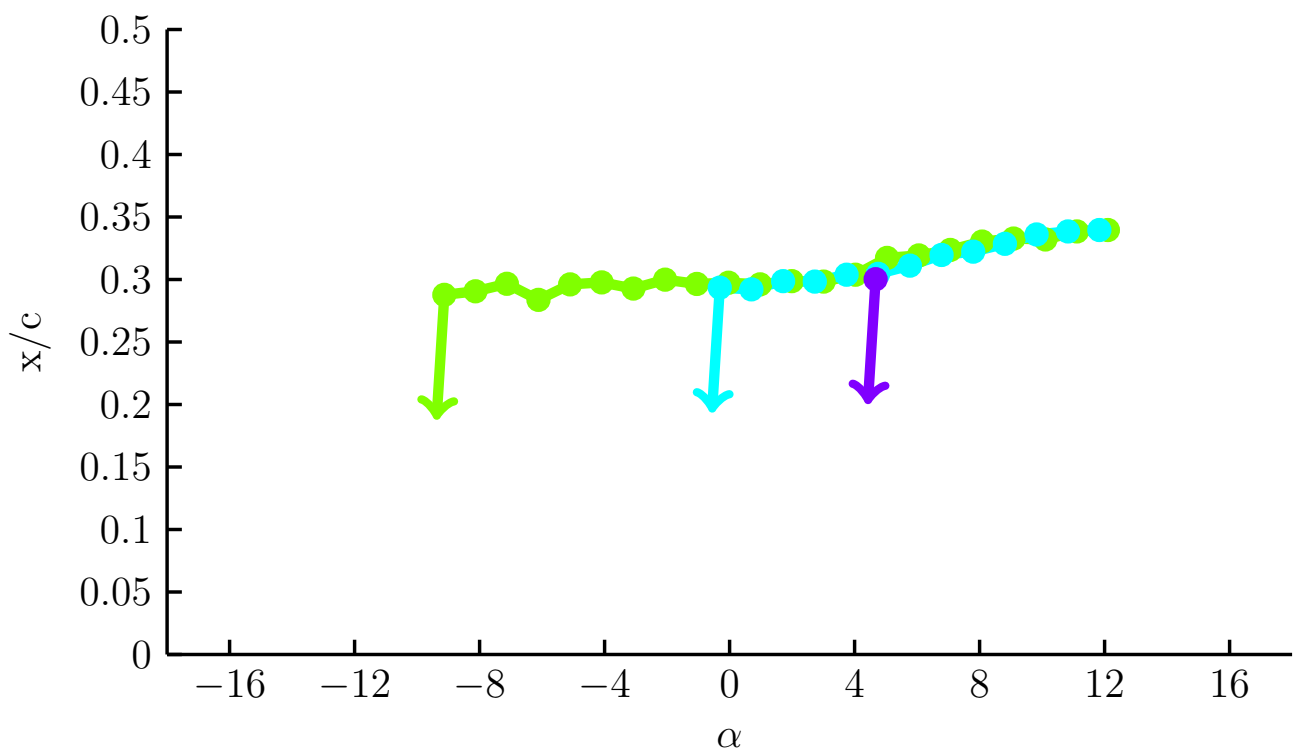

Figure III.33: Lower surface transition location variation with angle of attack for varying Reynolds number on the NREL S814 $100 \mu \mathrm{m}$ 03\% configuration. 


\section{CHAPTER IV}

\section{ANNUAL ENERGY PRODUCTION ANALYSIS}

Lift and drag forces on individual blade sections are responsible for generating the overall torque that rotates the turbine blades and produces power. One of the most common methods of analyzing the effects of airfoil characteristics on aerodynamic performance and power production is through blade-element-momentum (BEM) theory. BEM theory uses a differential element approach to combine 2D airfoil properties with a control volume power analysis. BEM can be used to determine power curves which can be combined with wind distributions to generate expected annual energy production (AEP). In this section, the BEM approach is used to calculate AEP for representative turbines using both the NACA $63_{3}-418$ and NREL S814 airfoils under a variety of wind and roughness conditions. Results are presented in terms of percent AEP loss for different roughness configurations, and results for the different airfoils are compared.

\section{IV.A. Blade-Element-Momentum Theory}

In momentum theory, a control volume is defined whose surfaces correspond to streamtubes spaced infinitesimally far apart and passing through the turbine blades. Two annular surfaces far upstream and far downstream of the rotor complete the control volume, as shown in Figure IV.1. At this stage the individual rotor blades are not considered, so the rotor is idealized as a "actuator disk". The actuator disk produces a force normal to its surface which is balanced by the change in pressure across the disk. Conservation of mass, linear momentum, angular momentum, and 
energy are written for the control volume and used to determine differential expressions for the normal force and torque produced by the actuator disk. The differential normal force, $d F_{n}$, and torque, $d T$, acting on a single annulus of the rotor disk are given by Equations 4.1 and 4.2:

$$
\begin{gathered}
d F_{n}=\rho U_{\infty}^{2}\left[4 a_{n}\left(1-a_{n}\right)\right] \pi r d r, \\
d T=4 \rho U_{\infty} \Omega\left[4 a_{t}\left(1-a_{n}\right)\right] \pi r^{3} d r,
\end{gathered}
$$

where $\rho$ is the air density, $U_{\infty}$ is the velocity upstream of the actuator disk, and $r$ is the radial position of the annulus from the center of the rotor disk.

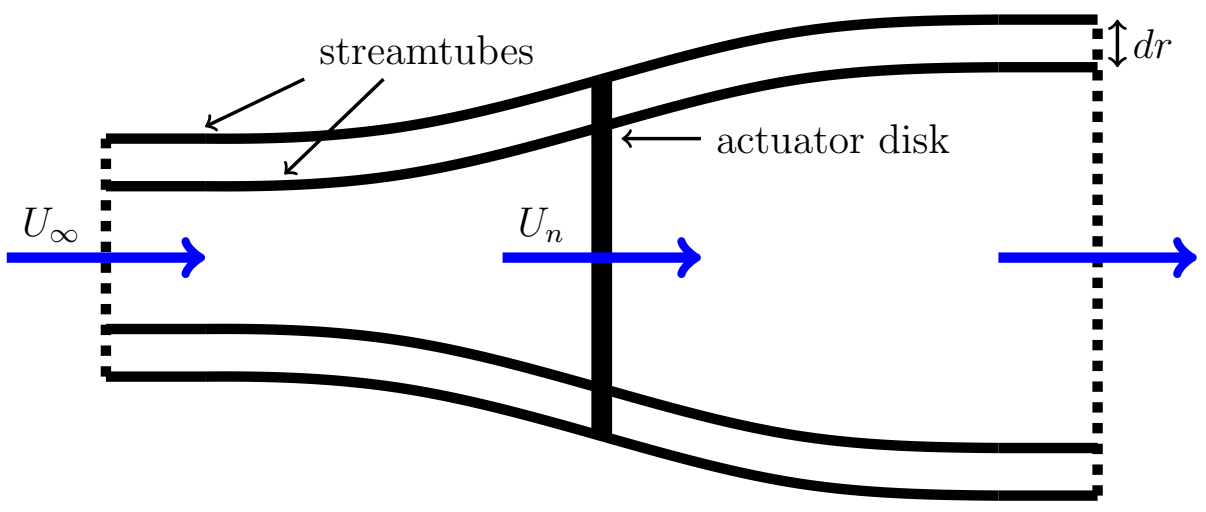

Figure IV.1: Momentum theory control volume.

The actuator disk induces flow velocities normal and tangential to the disk's rotation which are written in dimensionless form as normal and tangential induction factors, $a_{n}$ and $a_{t}$, respectively:

$$
\begin{gathered}
a_{n}=\frac{U_{\infty}-U_{n}}{U_{\infty}}, \\
a_{t}=\frac{U_{t}}{r \Omega},
\end{gathered}
$$


where $U_{n}$ is the normal velocity at the actuator disk. The tangential induction factor, characterized by the tangential velocity of the flow $U_{t}$ at the actuator disk and the disk's rotation rate $\Omega$, accounts for wake rotation.

In blade-element theory, the force and torque on each control volume annulus are determined by examining the individual blade elements in a blade-fixed frame. The velocities experienced by each blade section include axial and tangential components caused by freestream flow, blade rotation, and induction. The lift and drag forces are transformed into forces normal and tangential to the blade's rotation through a coordinate transformation involving the blade elements' pitch, angle of attack, and incoming flow angle $(\theta, \alpha$, and $\phi$, respectively). Figure IV.2 shows the various velocities, angles, and forces involved in the transformation. The flow angle can be determined through geometry and is dependent upon the axial and tangential induction factors, the blade section's spanwise location, and the ratio of rotational to axial flow. This ratio is characterized by the blade's tip-speed-ratio $X_{T S R}=R_{t i p} \Omega / U_{\infty}$, where, $R_{\text {tip }}$ is the radius of blade tip.

The lift, drag, normal, and tangential forces per unit span are written as $L^{\prime}$, $D^{\prime}, F_{n}^{\prime}$, and $F_{t}^{\prime}$. Airfoil performance characteristics are introduced into the expressions for $F_{n}^{\prime}$ and $F_{t}^{\prime}$ through normal and tangential force coefficients defined by $c_{n}=c_{l} \sin (\phi)+c_{d} \cos (\phi)$ and $c_{t}=c_{l} \cos (\phi)-c_{d} \sin (\phi)$, respectively. The normal forces and torques (tangential force multiplied by sectional radial location) from each blade are summed to arrive at the differential expressions given by Equations 4.5 and 4.6.

$$
d F_{n}=\sigma^{\prime} \pi \rho \frac{U_{\infty}^{2}\left(1-a_{n}\right)^{2}}{\cos ^{2}(\phi)} c_{n} r d r
$$




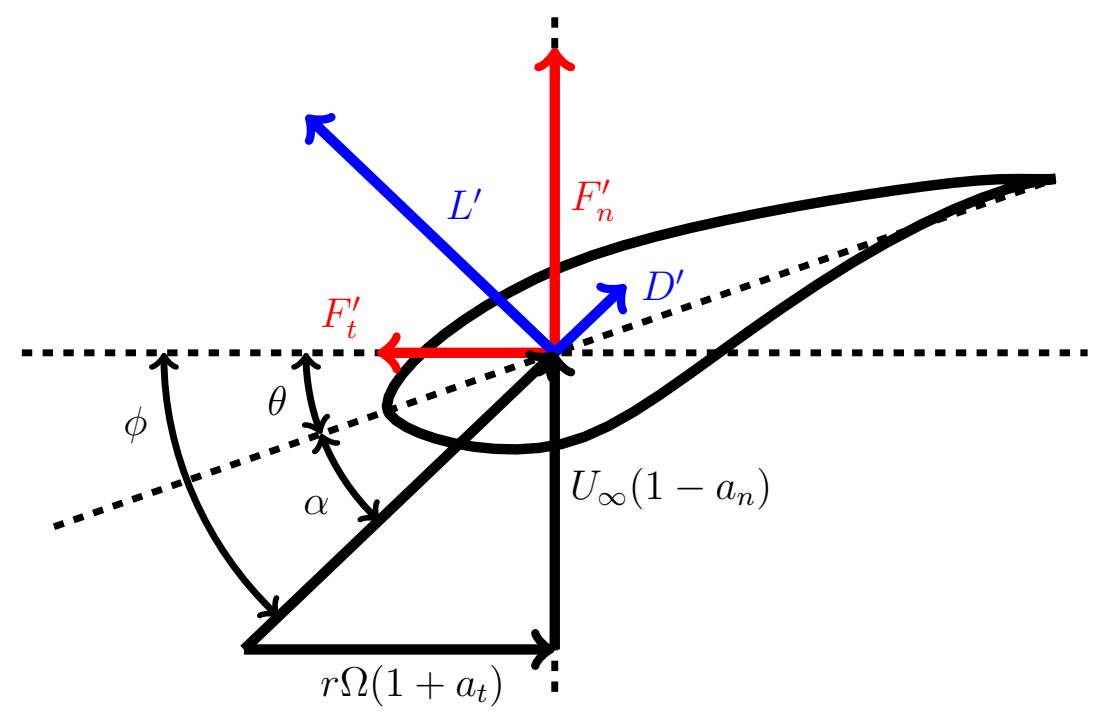

Figure IV.2: Turbine section velocity and force components.

$$
d T=\sigma^{\prime} \pi \rho \frac{U_{\infty}^{2}\left(1-a_{n}\right)^{2}}{\cos ^{2}(\phi)} c_{t} r^{2} d r
$$

where $\sigma^{\prime}=N_{b} c /(2 \pi r)$ is called the local blade solidity and is related to the number of blades, $N_{b}$, and the local blade chord length, $c$. The local solidity indicates the fractional amount of the rotor disk area that is covered by the blades. Finally, the expressions for axial and tangential force from the momentum theory and bladeelement theory approaches are equated to yield the fundamental equations of BEM theory:

$$
\begin{aligned}
\frac{a_{n}}{1-a_{n}} & =\frac{\sigma^{\prime} c_{n}}{4 \sin ^{2}(\phi)}, \\
\frac{a_{t}}{1-a_{n}} & =\frac{\sigma^{\prime} c_{t}}{4 \sin ^{2}(\phi)} .
\end{aligned}
$$

Equation 4.7 and 4.8 are solved iteratively to determine axial and tangential induction factors. These are used to solve for the total power of the turbine, which 
is nondimensionalized through the power coefficient, $C_{P}$ :

$$
C_{P}=\frac{P}{\frac{1}{2} \rho U_{\infty}^{3} S}
$$

where $P$ is the power produced by the turbine, $\rho$ is the air density, $U_{\infty}$ is the velocity upstream of the actuator disk, and $S$ is the actuator disk area.

Equation 4.9 cannot be solved directly since $P$ is not known. Instead, the differential power produced at each blade span location is written as the product of the tangential force and the local blade velocity. The total power is then determined by integration of these differential powers and nondimensionalized to arrive at Equation 4.10. The integration is performed from the hub radius, $R_{h u b}$, to the blade tip:

$$
C_{P}=\frac{X_{T S R}^{3}}{R_{t i p}^{4}} \int_{R_{\text {hub }}}^{R_{t i p}} \sigma^{\prime} c_{t} r^{3} d r
$$

\section{IV.B. Blade-Element-Momentum Corrections}

A major limitation of BEM theory is that the influence of vortices shed from the turbine tips is not considered. These vortices play a major role in modifying the induced velocity distribution of the rotor, especially near the blade tips. A commonly used correction which accounts for the vortices was developed by Prandtl [47]. The correction introduces a tip-loss factor $F_{t i p}$ which decreases the predicted normal force and torque from momentum theory for locations near the blade tip. The correction is expressed in Equation 4.11:

$$
F_{t i p}=\frac{2}{\pi} \cos ^{-1}\left(e^{-f_{t i p}}\right)
$$

where $f_{\text {tip }}=X_{T S R} N_{b}(R-r) /(2 r \sin (\phi))$. 
Rotors also experience vortex shedding from the hub. A correction factor with nearly identical form can also be applied to account for hub losses:

$$
F_{h u b}=\frac{2}{\pi} \cos ^{-1}\left(e^{-f_{h u b}}\right),
$$

where $f_{\text {hub }}=X_{T S R} N_{b}\left(r-R_{h u b}\right) /(2 r \sin (\phi))$. The total correction factor is written as the product of the hub and tip loss factors such that $F=F_{t i p} F_{h u b}$. These are multiplied on the right-hand-side of the momentum theory normal force and torque equations given by Equations 4.1 and 4.2 .

An additional problem with BEM theory occurs when induction factors become large and the turbine nears a turbulent wake state. The turbulent wake state occurs when $a_{n}$ is greater than 0.5 and, as predicted by momentum theory, the downstream wake reverses direction. In reality, this flow reversal cannot occur. Instead, more flow is entrained from outside the wake and turbulence increases [48]. A model recently used by Vaz is adopted to account for deviations from momentum theory caused by the turbulent wake state [49]. The model predicts that the rotor disk normal force should increase rather than decrease for large induction factors. This is implemented as a piecewise model where the infinitesimal normal force given by momentum theory is dependent upon the induction factor. The turbulent wake, root-loss, and tip-loss modifications to the momentum theory equations are given by Equations 4.13 and 4.14:

$$
d F_{n}= \begin{cases}\rho U_{\infty}^{2}\left[4 F a_{n}\left(1-a_{n}\right)\right] \pi r d r & : a_{n}<1 / 3, \\ \rho U_{\infty}^{2}\left[4 F a_{n}\left(1-\frac{a_{n}}{2}\left(5-3 a_{n}\right)\right)\right] \pi r d r & : a_{n} \geq 1 / 3,\end{cases}
$$


and

$$
d T=4 \rho U_{\infty} \Omega\left[4 F a_{t}\left(1-a_{n}\right)\right] \pi r^{3} d r
$$

These equations are set equal to the blade-element normal force and torque expressions to generate corrected versions of the fundamental BEM equations.

\section{IV.C. Blade-Element-Momentum Simulation}

The corrected BEM equations were used to calculate the power curves for three wind turbines: a turbine composed of NACA $63_{3}-418$ airfoils, a turbine composed of NREL S814 airfoils, and a turbine using both airfoils. The performance of each of these turbines was simulated for each of the roughness configurations described in Section III.C. The NREL 5 MW offshore turbine discussed in Section II.C was used as the geometry reference [39]. The turbine is a three-bladed, variable-speed, pitchcontrolled turbine rated for $5 \mathrm{MW}$ with an $11.4 \mathrm{~m} / \mathrm{s}$ rated wind speed. Cut-in and cut-out speeds are $3 \mathrm{~m} / \mathrm{s}$ and $25 \mathrm{~m} / \mathrm{s}$, respectively. The blade taper is approximately linear, and chord lengths range from $4.5 \mathrm{~m}$ near the hub to $1.5 \mathrm{~m}$ at the tip. The first $20 \%$ of the span is comprised of circular sections rather than airfoils. These sections produce no lift but increase the structural rigidity of the blade. Their drag coefficients are given by Jonkman [39]. The chord, twist, and airfoil distribution along the span is given in Appendix H.

The blade was discretized into 100 elements, and the induction factors for each element were calculated. Induction factor formulas were implicit, so an iterative approach was adopted. The calculations were repeated until changes in induction factor between iterations were less than $10^{-9}$. Airfoil lift and drag characteristics were 
implemented using lookup tables; angle of attack was calculated using $\alpha=\phi-\theta$ and linear interpolation was performed to determine the corresponding lift and drag from the experimental results in Section III.I.

BEM calculations require that the airfoil lift and drag polars are known over a large range of angle of attack, typically well past stall. Experimental test data only extends from $-16^{\circ}$ to $+16^{\circ}$ angle of attack, so the software QBlade was used to extrapolate lift and drag polars over the entire $360^{\circ}$ angle-of-attack range [50]. QBlade uses the Montgomerie extrapolation method which treats the flow as potential flow when the airfoil is near $0^{\circ}$ and $180^{\circ}$ angle of attack and as a stalled, thin plate at other angles of attack. A blending function is used between these two regimes to extrapolate polars for intermediate angles of attack [51]. Example $360^{\circ}$ lift and drag polars are shown in Figure IV.3.

In reality, insect distribution and Reynolds number change over the blade span. However, this was not implemented and all airfoil characteristics were taken for $R e_{c}=3.2 \times 10^{6}$ and for single roughness configurations. Results from these calculations should be used in a comparative sense only. For the combined NACA $63_{3}-418$ and NREL S814 airfoil case, blade regions where airfoil shape varied between the two airfoils were simulated by interpolating the airfoils' lift and drag polars so that smooth variation occurred along the span.

A control scheme was simulated for each turbine which maximizes the amount of wind power captured by the turbine. First, the tip-speed-ratio and pitch angle which maximized $C_{P}$ for each turbine's clean configuration was determined using a downhill simplex algorithm. When simulating operation in Region II of the power 


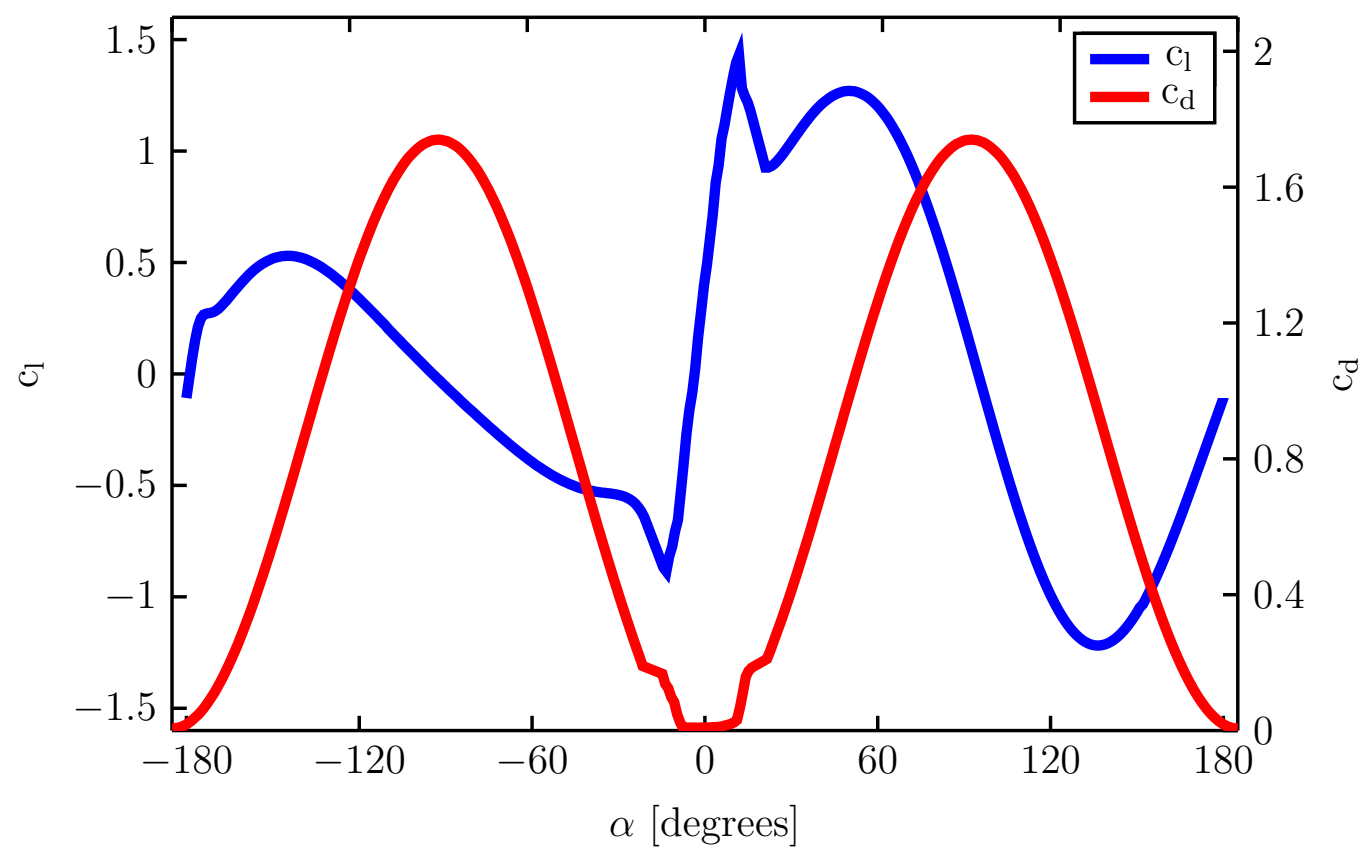

Figure IV.3: Example $360^{\circ}$ lift and drag polars. Polar corresponds to the NREL S814 clean configuration at $R e_{c}=3.2 \times 10^{6}$.

curve (between cut-in speed and rated speed), the tip-speed-ratio was set to this optimal value. Modern pitch controllers also search for the optimal pitch using PID control, so pitch was optimized in Region II using a golden section search. For the clean configurations, the calculated optimal pitch is identical to that given by the downhill simplex algorithm. However, each turbine's roughness configurations has slightly different optimal pitches due to airfoil performance losses. In Region III, the rotor rotational speed was set to its maximum value and pitch was set such that the turbine generates its rated power. Power coefficients were calculated at 64 wind speeds between the cut-in and cut-out speeds and used to generate power curves for each turbine and roughness combination. 


\section{IV.D. Annual Energy Production Calculations}

A map of the wind resource at $100 \mathrm{~m}$ above the ground is shown in Figure IV.4. The map shows high wind speeds in the central US and offshore, making these ideal locations for wind farms. IEC 61400-1 defines wind turbine classes by the mean wind speeds that the turbines are expected to experience [52]. Four wind turbine classes are specified: I, II, III, and IV with mean wind speeds of $10,8.5,7.5$, and $6.0 \mathrm{~m} / \mathrm{s}$, respectively. Wind speeds are typically assumed to follow Rayleigh distributions with probability density function $P D F$ and cumulative distribution function $C D F$ :

$$
\begin{gathered}
P D F=\frac{u}{\sigma^{2}} e^{-u^{2} /\left(2 \sigma^{2}\right),} \\
C D F=1-e^{-u^{2} /\left(2 \sigma^{2}\right)},
\end{gathered}
$$

where $u$ is the local wind speed and $\sigma$ is the shape factor, defined by the turbine class's mean wind speed $\bar{u}$ as $\sigma=\bar{u} \sqrt{2 / \pi}$.

The turbine's calculated power curves were integrated over the CDF to calculate the annual energy production. A factor of $N_{h}$, the number of hours in a year, is introduced to convert the power measurement into energy:

$$
A E P=N_{h} \int_{0}^{1} P d(C D F) .
$$

This integration is performed over the CDF which is itself a function of wind speed. $P$ is the power produced by the turbine at each wind speed, which is calculated through Equation 4.9 once the power coefficient for each speed is known. Alternatively, AEP can be calculated by integrating the product of power and PDF over the entire range of wind speeds and multiplying the result by $N_{h}$. 


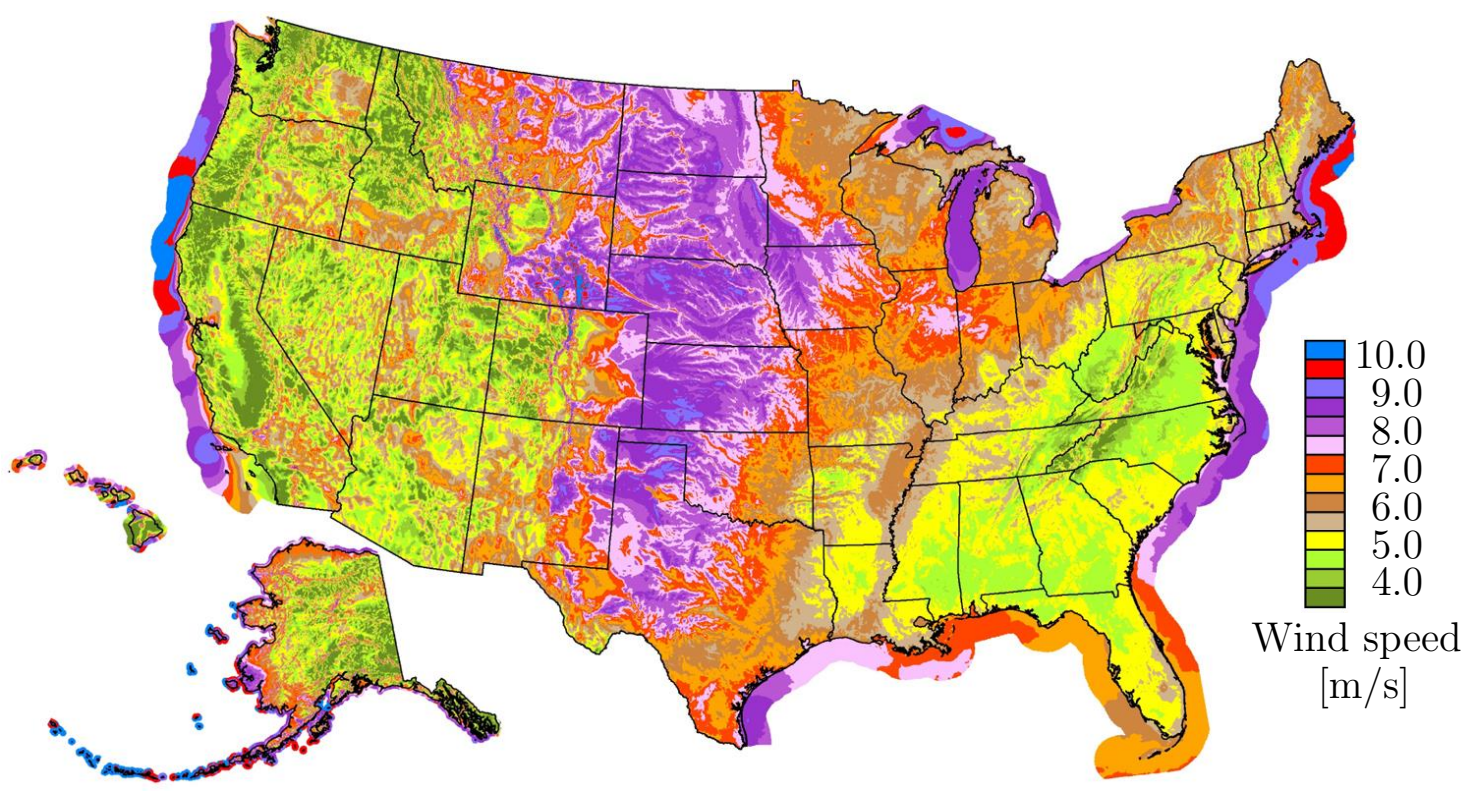

Figure IV.4: Map of U.S. wind resource. Map was developed by NREL using data from AWS Truepower [53].

\section{IV.E. Annual Energy Production Results}

Figure IV.5 shows the power losses for each configuration of the combined NACA $63_{3}-418$ and NREL S814 turbine. The Betz limit and clean power curve are plotted as solid and dashed black lines, respectively. The Betz limit represents the theoretical maximum power that can be extracted from a horizontal-axis wind turbine. It assumes that no viscous or tip losses are present and that the turbine is designed so that each turbine section operates at its ideal induction factor. No turbine can achieve the Betz limit, and power coefficients around $75 \%$ of this limit are typical in Region II, where turbines operate at their maximum power coefficient. The power coefficient is constant in this region due to modeling the airfoils at constant Reynolds number. Consequently, the power loss behaves with the same cubic shape as the total power in this region. In reality the power coefficient would 
likely decrease as wind velocity increases due to the increased effect of roughness at high Reynolds numbers. The power losses follows the same trends as airfoil lift-todrag ratios losses, with larger roughness heights and density corresponding to larger losses. For the clean case, the maximum $C_{P}$ is 0.443 . This is reduced to 0.411 for the $200 \mu \mathrm{m}$ roughness and even further reduced to 0.401 for the trip strip configuration. As seen in Section III.J, the trip strip configuration acts as a worst-case scenario for performance loss. For reference, the maximum $C_{P}$ given by Betz law is 0.593 , so the calculated values are typical of modern turbines.

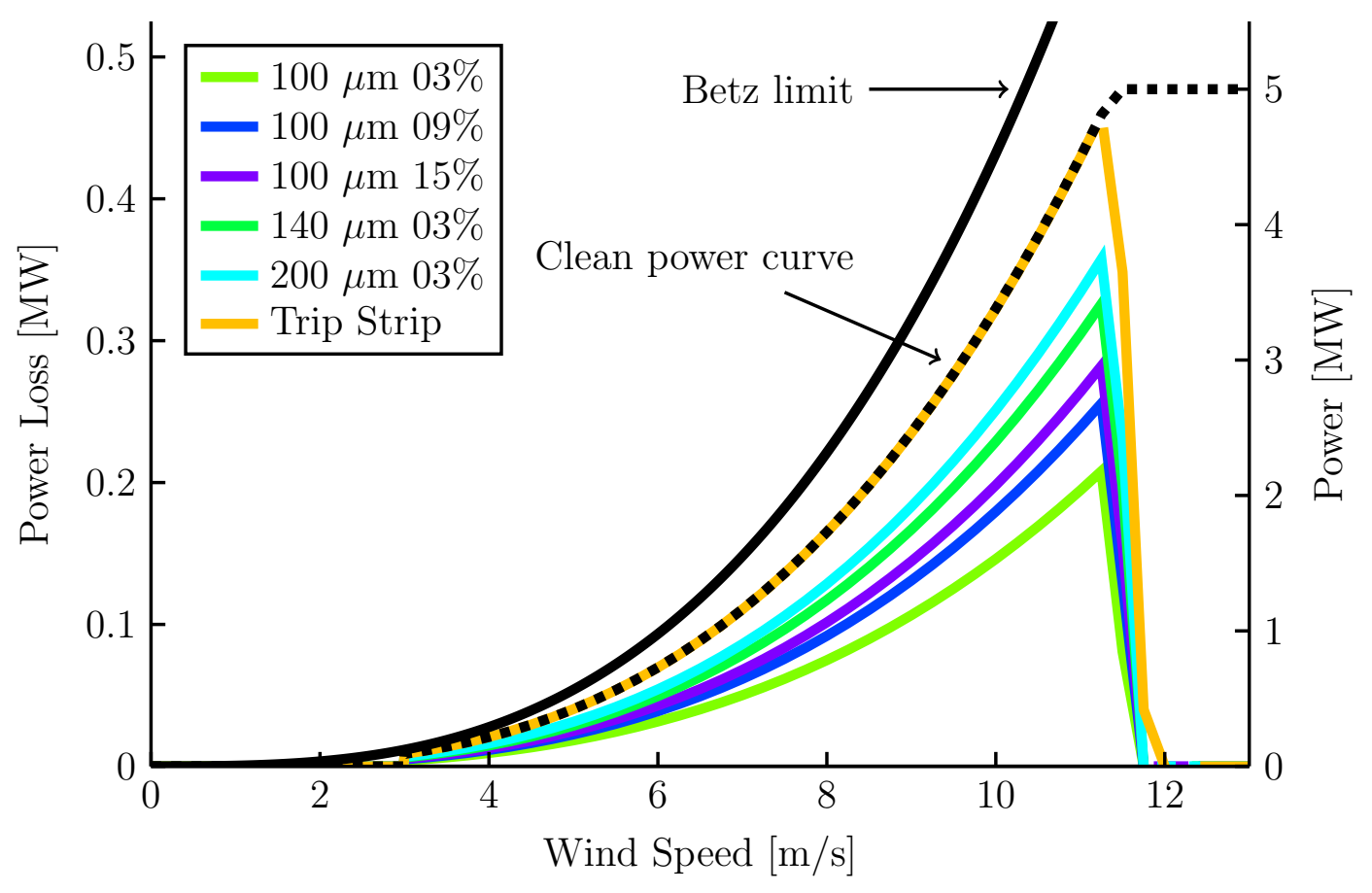

Figure IV.5: Power degradation plots. Plots correspond to the combined NACA $63_{3^{-}}$ 418 and NREL S814 turbine. Power curves (black lines) and power degradation curves (colored lines) follow the same trends in Region II of the power curve. 
An example of the AEP calculation method is given in Figure IV.6. Here, the clean power curve of the combined NACA $63_{3}-418$ and NREL S814 turbine is plotted with a dashed black line and overlaid with the PDF of the Rayleigh wind distributions. The AEP is calculated as the integral of these two functions, so maximum AEP loss occurs at wind speeds where both the PDF of wind speed and power loss are large. Percent AEP loss is maximized for Class IV wind speeds, but for most of the roughness configurations total AEP loss is greatest for Class II winds due to the larger base energy production at these speeds. Power loss is zero in Region III, so the wind classes which are concentrated in this region result in the smallest percent AEP loss.

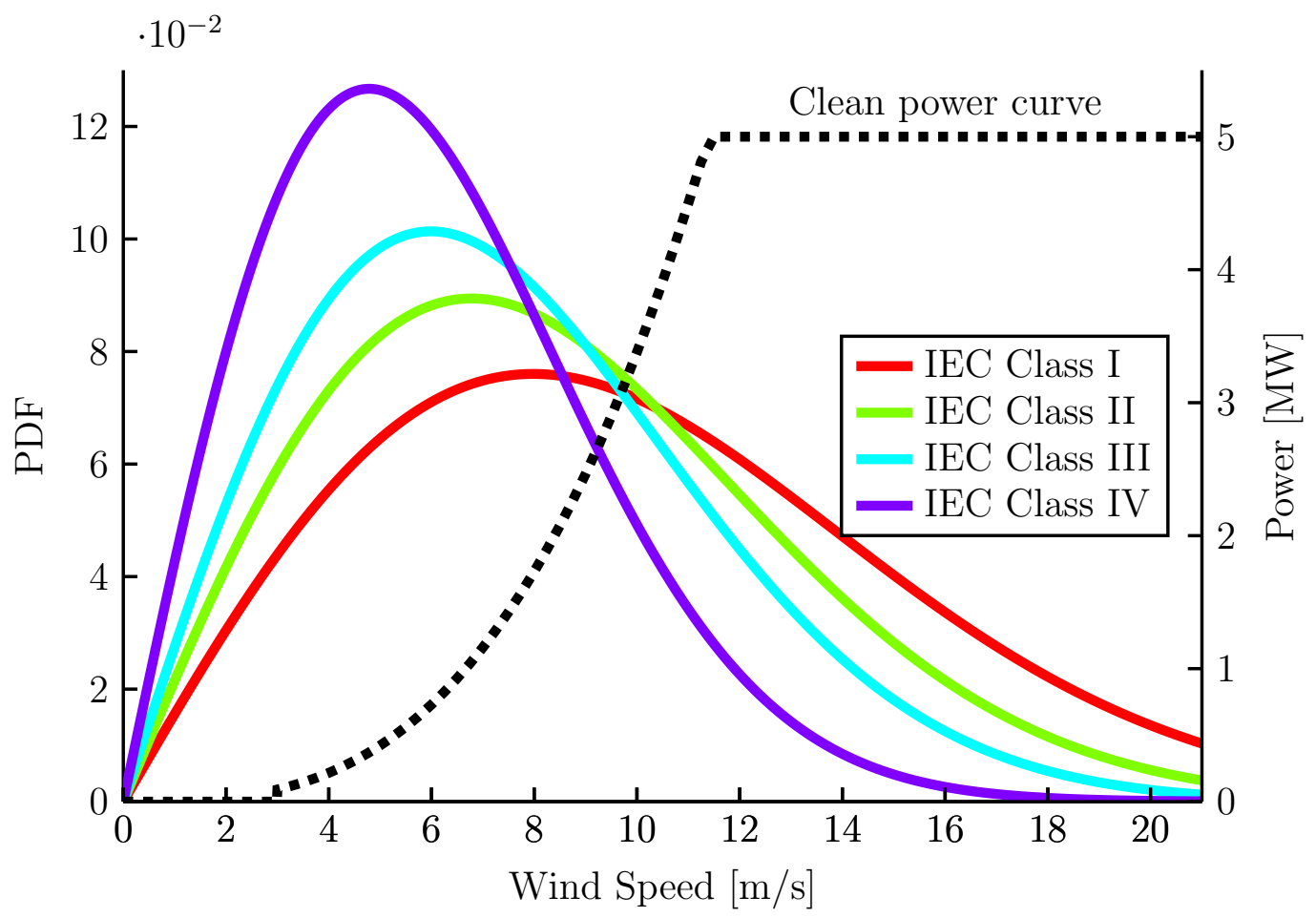

Figure IV.6: Rayleigh wind distribution plots with clean power curve overlay. Plots correspond to the combined NACA $63_{3}-418$ and NREL S814 turbine. 
Maximum power coefficient and AEP losses are summarized in Tables IV.1-IV.3. Losses are expressed as percent difference from the clean configuration values. The NACA $63_{3}-418$ turbine has the highest maximum $C_{P}$ and produces the most energy. Structural considerations limit the use of thin airfoils such as the NACA $63_{3}-418$ to outboard sections of the blade, however. The NREL S814 turbine has AEP losses which are $1 \%$ to $2 \%$ larger than the NACA $63_{3}-418$ turbine for the insect roughness cases, supporting the claim that thicker airfoils are more sensitive to roughness. For the trip strip case, differences in AEP loss are more pronounced with $3.7 \%$ difference in percent AEP loss between the NACA $63_{3}-418$ and NREL S814 turbines for Class IV wind speeds. The combined NREL S814 and NACA $63_{3}-418$ turbine shows performance which is intermediate between the two other turbines. The BEM method assumes that turbine sections are unaffected by the behavior of neighboring sections, so this is expected.

The amount of money lost due to insect contamination can be estimated by assuming a cost of energy of $\$ 0.05 / \mathrm{kW}$-hr and ideal operating conditions. For the clean case, each turbine would produce approximately $\$ 1,000,000$ annually for Class II winds. The presence of $200 \mu \mathrm{m}$ roughness would result in $\$ 30,000 ; \$ 43,000$; and $\$ 36,000$ annual losses for the NACA $63_{3}-418$, NREL S814, and combined turbines, respectively. These calculations assume that roughness is present year-round which is not typical. However, the calculation is still useful as a rough comparative tool between the turbines and provides an order-of-magnitude estimate of the performance impact. 
Table IV.1: AEP losses due to roughness on the NACA $63_{3}-418$ turbine.

\begin{tabular}{cccccc}
\hline \hline Configuration & $C_{P, \max }$ & IEC Class I & IEC Class II & IEC Class III & IEC Class IV \\
\hline Clean & 0.451 & 24.8 GW-hr & 20.3 GW-hr & 16.7 GW-hr & 10.7 GW-hr \\
$100 \mu \mathrm{m} \mathrm{03 \%}$ & $-3.2 \%$ & $-1.1 \%$ & $-1.5 \%$ & $-1.8 \%$ & $-2.4 \%$ \\
$100 \mu \mathrm{m} \mathrm{09 \%}$ & $-4.4 \%$ & $-1.6 \%$ & $-2.1 \%$ & $-2.5 \%$ & $-3.3 \%$ \\
$100 \mu \mathrm{m} \mathrm{15 \%}$ & $-5.5 \%$ & $-2.1 \%$ & $-2.7 \%$ & $-3.3 \%$ & $-4.3 \%$ \\
$140 \mu \mathrm{m} \mathrm{03 \%}$ & $-6.2 \%$ & $-2.4 \%$ & $-3.1 \%$ & $-3.8 \%$ & $-5.0 \%$ \\
$200 \mu \mathrm{m} \mathrm{03 \%}$ & $-6.1 \%$ & $-2.3 \%$ & $-3.0 \%$ & $-3.7 \%$ & $-4.9 \%$ \\
Trip Strip & $-8.0 \%$ & $-3.0 \%$ & $-3.9 \%$ & $-4.7 \%$ & $-6.2 \%$ \\
\hline \hline
\end{tabular}

Table IV.2: AEP losses due to roughness on the NREL S814 turbine.

\begin{tabular}{cccccc}
\hline \hline Configuration & $C_{P, \max }$ & IEC Class I & IEC Class II & IEC Class III & IEC Class IV \\
\hline Clean & 0.439 & $24.5 \mathrm{GW}-\mathrm{hr}$ & $20.0 \mathrm{GW}-\mathrm{hr}$ & $16.5 \mathrm{GW}-\mathrm{hr}$ & $10.5 \mathrm{GW}-\mathrm{hr}$ \\
$100 \mu \mathrm{m} \mathrm{03 \%}$ & $-6.3 \%$ & $-2.4 \%$ & $-3.1 \%$ & $-3.7 \%$ & $-4.9 \%$ \\
$100 \mu \mathrm{m} \mathrm{09 \%}$ & $-6.3 \%$ & $-2.4 \%$ & $-3.1 \%$ & $-3.7 \%$ & $-4.9 \%$ \\
$100 \mu \mathrm{m} \mathrm{15 \%}$ & $-6.5 \%$ & $-2.4 \%$ & $-3.2 \%$ & $-3.8 \%$ & $-5.0 \%$ \\
$140 \mu \mathrm{m} \mathrm{03 \%}$ & $-6.7 \%$ & $-2.5 \%$ & $-3.3 \%$ & $-4.0 \%$ & $-5.2 \%$ \\
$200 \mu \mathrm{m} \mathrm{03 \%}$ & $-8.2 \%$ & $-3.3 \%$ & $-4.3 \%$ & $-5.2 \%$ & $-6.8 \%$ \\
Trip Strip & $-12.6 \%$ & $-4.9 \%$ & $-6.4 \%$ & $-7.6 \%$ & $-9.9 \%$ \\
\hline \hline
\end{tabular}

Table IV.3: AEP losses due to roughness on the combined NACA $63_{3}-418$ and NREL S814 turbine.

\begin{tabular}{cccccc}
\hline \hline Configuration & $C_{P, \max }$ & IEC Class I & IEC Class II & IEC Class III & IEC Class IV \\
\hline Clean & 0.443 & 24.6 GW-hr & 20.1 GW-hr & 16.5 GW-hr & 10.6 GW-hr \\
$100 \mu \mathrm{m} \mathrm{03 \%}$ & $-4.3 \%$ & $-1.6 \%$ & $-2.1 \%$ & $-2.5 \%$ & $-3.3 \%$ \\
$100 \mu \mathrm{m} \mathrm{09 \%}$ & $-5.2 \%$ & $-2.0 \%$ & $-2.6 \%$ & $-3.1 \%$ & $-4.1 \%$ \\
$100 \mu \mathrm{m} \mathrm{15 \%}$ & $-5.7 \%$ & $-2.2 \%$ & $-2.8 \%$ & $-3.4 \%$ & $-4.5 \%$ \\
$140 \mu \mathrm{m} \mathrm{03 \%}$ & $-6.6 \%$ & $-2.5 \%$ & $-3.3 \%$ & $-4.0 \%$ & $-5.2 \%$ \\
$200 \mu \mathrm{m} \mathrm{03 \%}$ & $-7.2 \%$ & $-2.8 \%$ & $-3.6 \%$ & $-4.4 \%$ & $-5.7 \%$ \\
Trip Strip & $-9.5 \%$ & $-3.6 \%$ & $-4.7 \%$ & $-5.7 \%$ & $-7.4 \%$ \\
\hline \hline
\end{tabular}




\section{CHAPTER V}

\section{CONCLUSIONS \& FUTURE WORK}

This dissertation combined insect impingement prediction tools, wind tunnel testing, and BEM calculations to quantify the effect of insect roughness on wind

turbine performance for two representative airfoils. Wind farm operators and financiers frequently cite lower-than-predicted energy production, much of which may be attributable to insect-induced power losses. Past roughness sensitivity studies have used roughness modeling techniques which either do not accurately represent insect roughness or are expensive and difficult to reproduce. This work provides a test method that is both accurate and cost-effective. The technique can be adopted directly to account for roughness during blade design or used to validate computational roughness models. The use of computational roughness models will allow for more accurate wind plant performance predictions and will enable design innovation to mitigate roughness effects.

First, an insect impingement code called LEWBUG was written and used to predict insect impingement patterns on turbine blades. Insect patterns were found to rely on insect mass and drag parameters which vary between species. The housefly was found to be representative of the insect population and used to quantify the expected impingement patterns. Multiple airfoils were tested to determine the effect of airfoil thickness on insect distribution. It was found that thicker airfoils exhibit increased rates of impingement and larger impact velocities (a quantity that determines whether insects will adhere to a surface after impact). Accordingly, a $24 \%$ 
thick NREL S814 airfoil was chosen for roughness-sensitivity wind tunnel testing to act as a counterpart to similar tests of the NACA $63_{3}-418$ conducted by Ehrmann [7].

Wind tunnel testing was conducted at $R e_{c}=1.6 \times 10^{6}, 2.4 \times 10^{6}, 3.2 \times 10^{6}$, and $4.0 \times 10^{6}$ using vinyl decals to simulate insect roughness. Seven roughness configu-

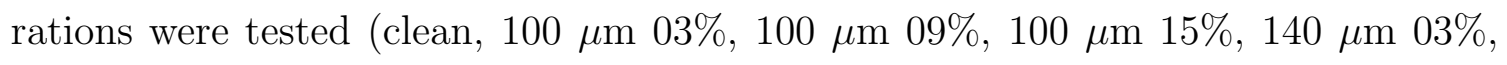
$200 \mu \mathrm{m} 03 \%$, and trip strip) with roughness locations determined by the insect impingement code. Lift, pitching moment, and drag results were obtained by pressure measurements. Transition locations were determined using infrared thermography.

Pressure measurements indicate that the presence of roughness decreases maximum lift and lift curve slope, and increases drag. Performance degradation was increased when roughness height and density was increased, with roughness height having a larger effect than density. Performance losses for the NREL S814 at positive angles of attack were similar to those observed by Ehrmann, with a loss in maximum lift-to-drag ratio of $38.0 \%$ for the $200 \mu \mathrm{m}$ roughness (compared to $36.6 \%$ on Ehrmann's NACA $63_{3}-418$ ). Behavior at low angles of attack was vastly different for the NREL S814 than the NACA $63_{3}-418$. The NREL S814 exhibited premature lower-surface stall which increased the lower-surface stall angle of attack by $8 \%$ for the $200 \mu \mathrm{m}$ roughness. Differences in lower surface performance are likely due to the added thickness and correspondingly larger pressure gradients on this surface.

Increased roughness height and density resulted in decreased bypass angles of attack on the upper airfoil surface and increased bypass angles of attack on the lower surface. This was consistent between the NACA $63_{3}-418$ and NREL S814 airfoils. On the NREL S814 airfoil's lower surface, transition location varied little with angle 
of attack because of the large adverse pressure gradient near the point of maximum airfoil thickness. For all surfaces, bypass occurred within the expected range of critical roughness Reynolds numbers.

Airfoil performance data was used to simulate soiled turbines with a BEM code. AEP losses corresponded directly to airfoil performance losses, with the largest AEP losses occurring for the largest roughness heights and densities. Turbines were simulated using the NREL 5 MW reference turbine properties with blade sections consisting entirely of NACA $63_{3}-418$ airfoils, entirely of NREL S814 airfoils, and using a combined profile. The NREL S814 turbine exhibited maximum $C_{P}$ losses due to roughness of $8.2 \%$ compared to $6.1 \%$ for the NACA $63_{3}-418$ turbine. Maximum percent AEP losses $\left(4.9 \%, 6.8 \%\right.$, and $5.7 \%$ for the NACA $63_{3}-418$, NREL S814, and combined turbines, respectively) occurred for the lowest-speed wind class. Performance losses translate to $\$ 30,000, \$ 43,000$, and $\$ 36,000$ annual losses for the three turbines in the Class II wind regime.

The study successfully quantified the roughness sensitivity of the two airfoils. Moving forward, more blades should be tested of varying thicknesses to further isolate the effect of thickness. Camber varied significantly between these airfoils, so the role of thickness itself cannot be completely determined.

More precise modeling of insect collection patterns should also be conducted during wind tunnel testing. This data is easily obtainable through LEWBUG but was utilized only to determine insect chordwise impingement limits. Variable-density insect patterns could be used to better model roughness. 
Collection patterns from LEWBUG could also be implemented with computational fluid dynamics software to create a rapid airfoil design tool. A viscous-inviscid interaction (VII) scheme which combines panel methods and boundary layer solvers would be ideal for this function as both LEWBUG and panel method solvers are computational inexpensive. These could be combined with bypass transition models such as critical roughness Reynolds number correlations to predict the performance effect of insect roughness. This tool would allow designers to tailor their airfoils to reduce both insect collection and sensitivity to roughness. 


\section{REFERENCES}

[1] Boccard, N., "Capacity factor of wind power realized values vs. estimates," Energy Policy, Vol. 37, No. 7, 2009, pp. 2679-2688.

[2] Albers, A. and Gerdes, G., "Wind farm performance verification," DEWIMagazin, Vol. 8, No. 14, 1999, pp. 24-35.

[3] White, E., Kutz, D., Freels, J., Hidore, J., Grife, R., Sun, Y., and Chao, D., "Leading-edge roughness effects on 633-418 airfoil performance," AIAA Paper, AIAA, Vol. 352, 2011.

[4] Standish, K., Rimmington, P., Laursen, J., Paulsen, H., and Nielsen, D., "Computational prediction of airfoil roughness sensitivity," AIAA Paper, AIAA, Vol. 460, 2010.

[5] Corten, G. and Veldkamp, H., "Aerodynamics: Insects can halve wind-turbine power," Nature, Vol. 412, No. 6842, 2001, pp. 41-42.

[6] Spruce, C., "Power performance of active stall wind turbines with blade contamination," European Wind Energy Conference, Athens, 2006.

[7] Ehrmann, R., Effect of Surface Roughness on Wind Turbine Performance, Ph.D. thesis, Texas A\&M University, College Station, TX, USA, 2015.

[8] Moroz, E. and Eggleston, D., "A comparison between actual insect contamination and its simulation," Wind Power, Vol. 7, 1992, pp. 418-425. 
[9] Tangler, J., Smith, B., and Jager, D., "SERI advanced wind turbine blades," Tech. Rep. TP-257-4492, National Renewable Energy Laboratory, 1992.

[10] Boermans, L. and Selen, H., "On the design of some airfols for sailplane application," LR-326, Delft University of Technology, Delft, Netherlands, 1981.

[11] Coleman, W., "The characteristics of roughness from insects as observed for twodimensional, incompressible flow past airfoils," Journal of Aerospace Sciences, Vol. 26, No. 5, 1959, pp. 264-280.

[12] Abbott, I., van Doenhoff, A., and Stivers, L., "Summary of airfoil data," TR 824, NACA, Langley Field, VA, USA.

[13] Soltani, M., Birjandi, A., and Seddighi Moorani, M., "Effect of surface contamination on the performance of a section of a wind turbine blade," Scientia Iranica, Vol. 18, No. 3, 2011, pp. 349-357.

[14] Tangler, J. and Somers, D., "NREL airfoil families for HAWTs," TP-442-7109, National Renewable Energy Laboratory, Golden, CO, USA, 1995.

[15] Eppler, R., Airfoil Design and Data, Springer Science \& Business Media, 1990.

[16] Sareen, A., Sapre, C., and Selig, M., "Effects of leading edge erosion on wind turbine blade performance," Wind Energy, Vol. 17, No. 10, 2014, pp. 1531-1542.

[17] Timmer, W. and Schaffarczyk, A., "The effect of roughness at high Reynolds numbers on the performance of aerofoil DU 97-W-300Mod," Wind Energy, Vol. 7, No. 4, 2004, pp. 295-307. 
[18] Tangler, J., Smith, B., Jager, D., and Olsen, T., "SERI thin-airfoil blade atmospheric performance test: Final results," Windpower 90, 1990, pp. 118-125.

[19] Powers, J., "Promising results from new blades," WindStats, 1990.

[20] Janiszewska, J., Ramsay, R., Hoffmann, M., and Gregorek, G., "Effects of grit roughness and pitch oscillations on the S814 airfoil," TP-442-8161, National Renewable Energy Laboratory, Golden, CO, USA, 1996.

[21] Ramsay, R., Hoffman, M., and Gregorek, G., "Effects of grit roughness and pitch oscillations on the S815 airfoil," Tech. Rep. TP-442-7820, National Renewable Energy Laboratory, Golden, CO, USA, 1996.

[22] Somers, D. and Tangler, J., "Wind tunnel test of the S814 thick root airfoil," Journal of Solar Energy Engineering, Vol. 118, No. 4, 1996, pp. 217-221.

[23] Timmer, W. and Van Rooij, R., "Summary of the Delft University wind turbine dedicated airfoils," Journal of Solar Energy Engineering, Vol. 125, No. 4, 2003, pp. $488-496$.

[24] Drela, M. and Youngren, H., XFOIL 6.9 User Guide, 2011 (accessed November 14, 2014).

[25] Van Rooij, R. and Timmer, W., "Roughness sensitivity considerations for thick rotor blade airfoils," Journal of Solar Energy Engineering, Vol. 125, No. 4, 2003, pp. $468-478$.

[26] Morkovin, M., "Bypass transition to turbulence and research desiderata," In NASA Lewis Research Center Transition in Turbines, Vol. 1, 1985, pp. 161-204. 
[27] Dryden, H., "Review of published data on the effect of roughness on transition from laminar to turbulent flow," Journal of the Aeronautical Sciences, 2012.

[28] Smith, A., "The smallest height of roughness capable of affecting boundary-layer transition," Journal of the Aerospace Sciences, 2012.

[29] Schiller, L., "Strömung in rohren," Handbuch Der Experimentalphysik, Vol. 4, Akad. Verlagsgesellschaft, Leipzig, 1932, pp. 189-192.

[30] Tani, I., "Boundary-layer transition," Annual Review of Fluid Mechanics, Vol. 1, No. 1, 1969, pp. 169-196.

[31] Downs, R., White, E., and Denissen, N., "Transient growth and transition induced by random distributed roughness," AIAA journal, Vol. 46, No. 2, 2008, pp. $451-462$.

[32] Johnson, D., "Brief measurements of insect contamination on aircraft wings," TR-2164, RAE, 1952.

[33] Maresh, J. and Bragg, M., "The role of airfoil geometry in minimizing the effect of insect contamination of laminar flow sections," AIAA 2nd Applied Aerodynamics Conference, Seattle, Washington, 1984.

[34] Bragg, M., Rime ice accretion and its effect on airfoil performance., Ph.D. thesis, Ohio State University, Columbus, Ohio, USA, 1982.

[35] Miller, D., Bond, T., Sheldon, D., Wright, W., Langhals, T., Al-Khalil, K., and Broughton, H., "Validation of NASA thermal ice protection computer codesPart 1: Program overview," TM-107397, NASA, Cleveland, OH, USA, 1997. 
[36] Hess, J. and Smith, A., "Calculation of potential flow about arbitrary bodies," Progress in Aerospace Sciences, Vol. 8, 1967, pp. 1-138.

[37] Tucker, V., "Energetic cost of locomotion in animals," Comparative Biochemistry and Physiology, Vol. 34, No. 4, 1970, pp. 841-846.

[38] Vogel, S., Life in Moving Fluids, Princeton University Press, Princeton, New Jersey, 1994.

[39] Jonkman, J., Butterfield, S., Musial, W., and Scott, G., "Definition of a 5MW reference wind turbine for offshore system development," TP-500-38060, National Renewable Energy Laboratory, Golden, CO, USA, 2009.

[40] Krishnan, K., Robison, R., Tetteh, E., Loth, E., Farrell, T., Crouch, J., and Berry, D., "Characterization of insect residue on an aerodynamic leading edge," 8th AIAA Atmospheric and Space Environments Conference, 2016, p. 3445.

[41] Timmer, W. and van Rooy, R., "Thick airfoils for HAWTs," Journal of Wind Engineering and Industrial Aerodynamics, Vol. 39, No. 1, 1992, pp. 151-160.

[42] Wilcox, B. and White, E., "Computational analysis of insect impingement patterns on wind turbine blades," Wind Energy, 2015.

[43] Bearman, P., "Corrections for the effect of ambient temperature drift on hotwire measurements in incompressible flow," DISA information, Vol. 11, No. 1, 1971, pp. 25-30. 
[44] Ehrmann, R. and White, E., "Influence of 2D steps and distributed roughness on transition on a NACA 633-418," 2nd ASME Wind Energy Symposium, 2014, pp. $13-17$.

[45] Barlow, J., Rae, W., and Pope, A., Low-Speed Wind Tunnel Testing, John Wiley \& Sons, Inc, 1999.

[46] Selig, M. and McGranahan, B., "Wind tunnel aerodynamic tests of six airfoils for use on small wind turbines," Journal of Solar Energy Engineering, Vol. 126, No. 4, 2004, pp. 986-1001.

[47] Glauert, H., "Airplane propellers," Aerodynamic Theory, Springer, 1935, pp. 169-360.

[48] Moriarty, P. and Hansen, A., "AeroDyn theory manual," TP-500-36881, National Renewable Energy Laboratory, Golden, CO, USA, 2005.

[49] Vaz, J., Pinho, J., and Mesquita, A., "An extension of BEM method applied to horizontal-axis wind turbine design," Renewable Energy, Vol. 36, No. 6, 2011, pp. $1734-1740$.

[50] Marten, D., Wendler, J., Pechlivanoglou, G., Nayeri, C., and Paschereit, C., "Qblade: an open source tool for design and simulation of horizontal and vertical axis wind turbines," International Journal of Emerging Technology and Advanced Engineering (IJETAE), Vol. 3, 2013, pp. 264-269.

[51] Montgomerie, B., "Methods for root effects, tip effects and extending the angle of attack range to \pm 180 , with application to aerodynamics for blades on wind 
turbines and propellers," R-1035-SE, FOI Swedish Defense Research Agency, Stockholm, Sweden, 2004.

[52] TC88-MT, I., "IEC 61400-3: Wind turbines-Part 1: Design requirements," International Electrotechnical Commission, 2005.

[53] Elliott, D. and Schwartz, M. N., "Development and validation of high-resolution state wind resource maps for the United States," TP-500-38127, National Renewable Energy Laboratory, Golden, CO, USA, 2005. 


\section{APPENDIX A}

\section{AIRFOIL COORDINATES}

NREL S814 airfoil coordinates.

\begin{tabular}{cccc}
\hline \hline$x / c$ & $y / c$ & $x / c$ & $y / c$ \\
\hline 1.0000 & 0.0000 & 0.0000 & 0.0000 \\
0.9963 & 0.0011 & 0.0005 & -0.0047 \\
0.9858 & 0.0047 & 0.0061 & -0.0175 \\
0.9696 & 0.0107 & 0.0164 & -0.0316 \\
0.9488 & 0.0185 & 0.0310 & -0.0465 \\
0.9238 & 0.0271 & 0.0492 & -0.0616 \\
0.8946 & 0.0357 & 0.0708 & -0.0766 \\
0.8610 & 0.0442 & 0.0952 & -0.0910 \\
0.8231 & 0.0528 & 0.1219 & -0.1041 \\
0.7817 & 0.0615 & 0.1507 & -0.1155 \\
0.7372 & 0.0702 & 0.1812 & -0.1243 \\
0.6904 & 0.0787 & 0.2132 & -0.1297 \\
0.6417 & 0.0870 & 0.2471 & -0.1308 \\
0.5920 & 0.0947 & 0.2839 & -0.1274 \\
0.5418 & 0.1017 & 0.3239 & -0.1199 \\
0.4917 & 0.1078 & 0.3675 & -0.1089 \\
0.4424 & 0.1125 & 0.4148 & -0.0951 \\
0.3944 & 0.1157 & 0.4655 & -0.0796 \\
0.3483 & 0.1170 & 0.5191 & -0.0633 \\
0.3042 & 0.1156 & 0.5749 & -0.0470 \\
0.2618 & 0.1118 & 0.6319 & -0.0317 \\
0.2211 & 0.1058 & 0.6891 & -0.0182 \\
0.1827 & 0.0982 & 0.7453 & -0.0070 \\
0.1469 & 0.0892 & 0.7990 & 0.0013 \\
0.1141 & 0.0790 & 0.8489 & 0.0067 \\
0.0848 & 0.0679 & 0.8935 & 0.0092 \\
0.0592 & 0.0561 & 0.9315 & 0.0091 \\
0.0377 & 0.0438 & 0.9620 & 0.0070 \\
0.0206 & 0.0313 & 0.9836 & 0.0038 \\
0.0083 & 0.0189 & 0.9961 & 0.0010 \\
0.0012 & 0.0070 & 1.0000 & 0.0000 \\
\hline \hline
\end{tabular}




\section{APPENDIX B}

\section{FLOW UNIFORMITY SUMMARY}

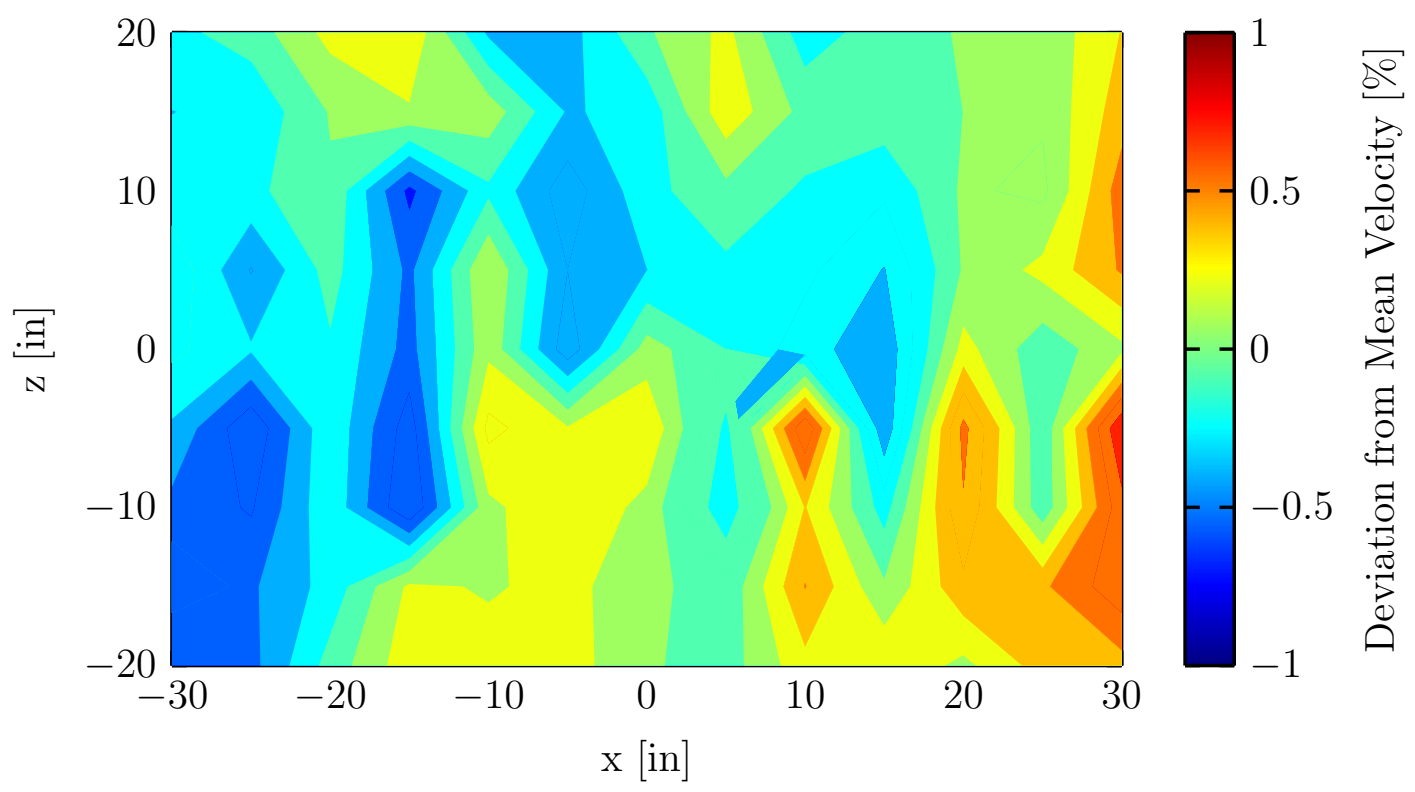

Flow uniformity plot at $R e_{c}=1.6 \times 10^{6}$. 


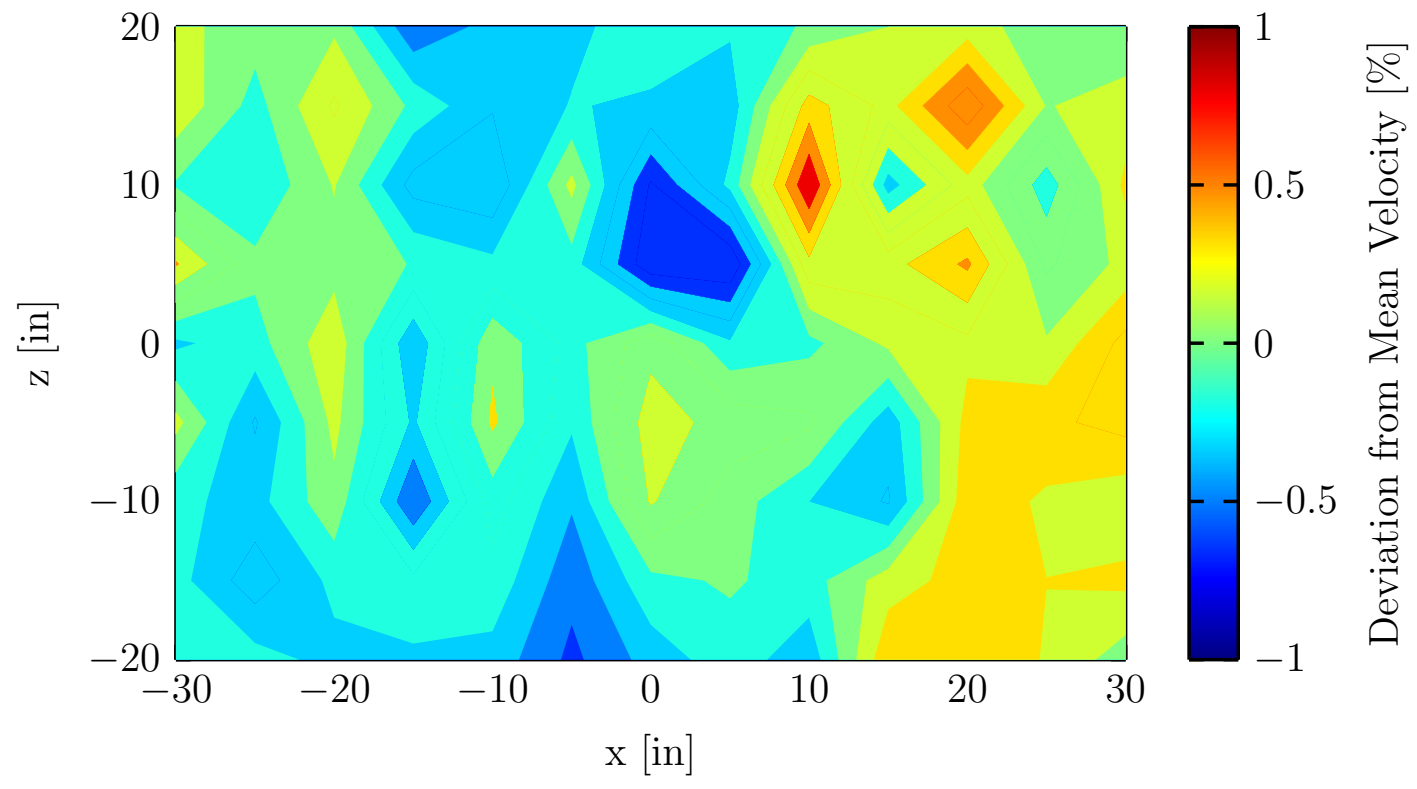

Flow uniformity plot at $R e_{c}=2.4 \times 10^{6}$. 


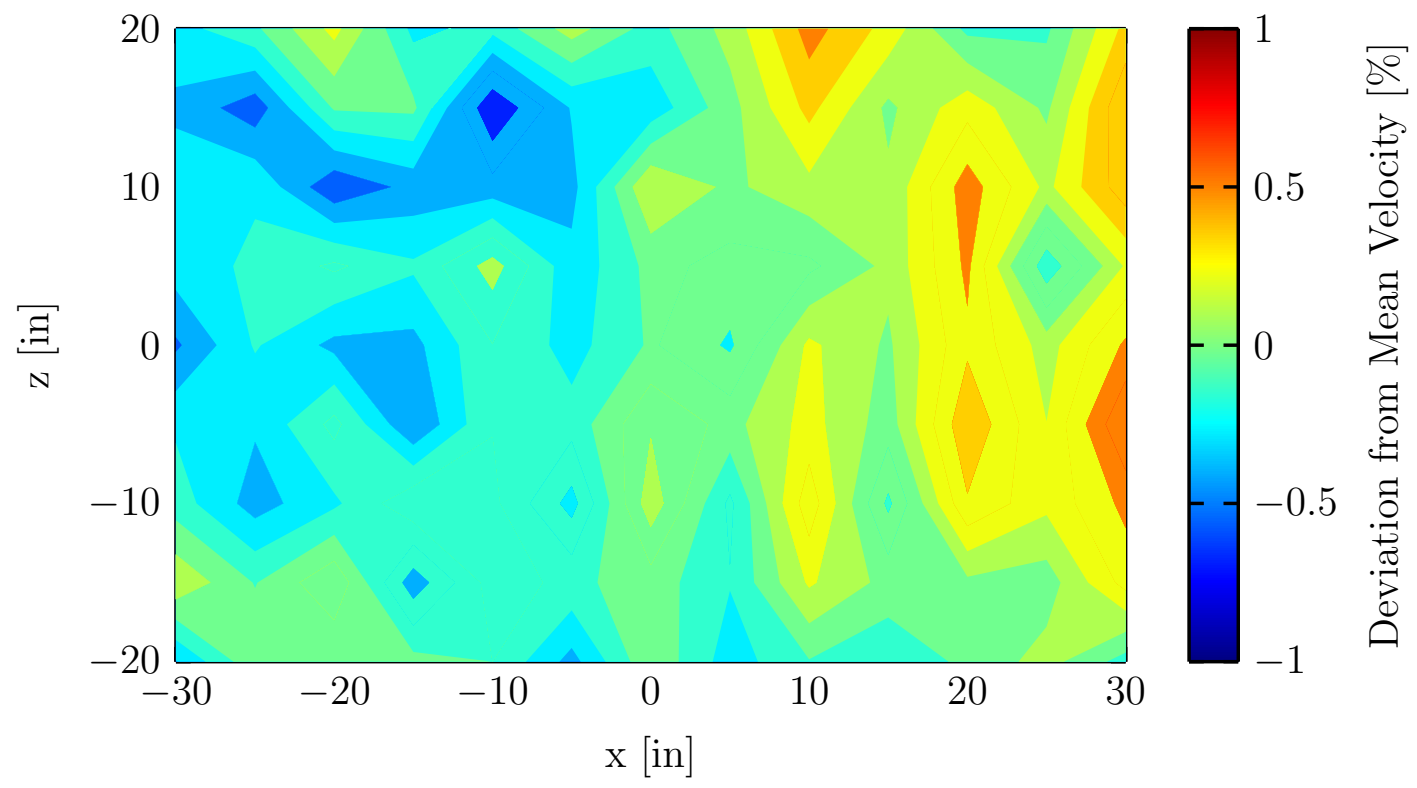

Flow uniformity plot at $R e_{c}=3.2 \times 10^{6}$. 


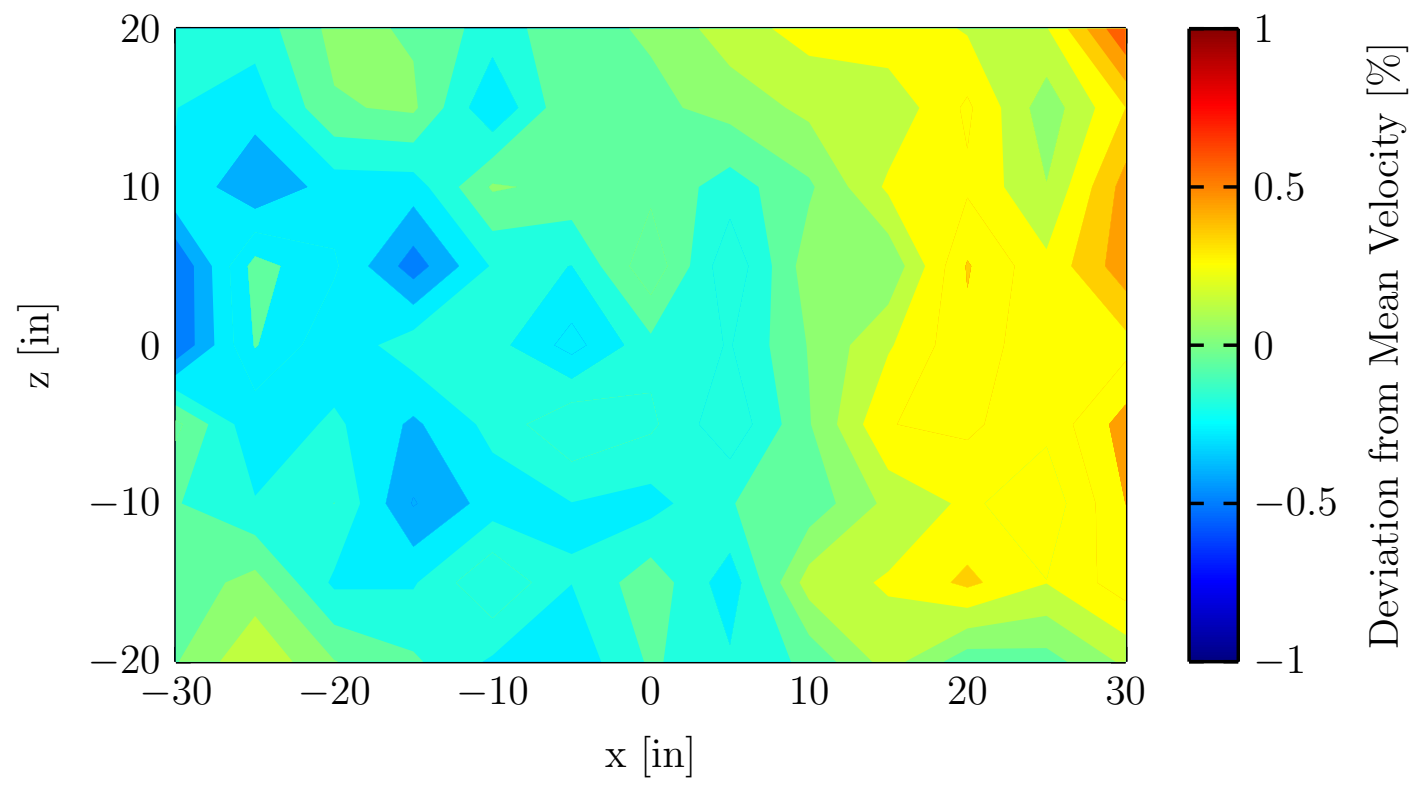

Flow uniformity plot at $R e_{c}=4.0 \times 10^{6}$. 


\section{APPENDIX C}

\section{PRESSURE TAP COORDINATES}

NREL S814 pressure tap locations.

\begin{tabular}{cccccc}
\hline \hline$x / c$ & $y / c$ & $z / c$ & $x / c$ & $y / c$ & $z / c$ \\
\hline 0.9492 & 0.0191 & 0.0000 & 0.0069 & -0.0187 & 0.2735 \\
0.8989 & 0.0345 & 0.0144 & 0.0203 & -0.0359 & 0.2793 \\
0.8478 & 0.0472 & 0.0288 & 0.0361 & -0.0510 & 0.2850 \\
0.7964 & 0.0584 & 0.0432 & 0.0532 & -0.0646 & 0.2908 \\
0.7448 & 0.0687 & 0.0576 & 0.0712 & -0.0769 & 0.2965 \\
0.6930 & 0.0783 & 0.0720 & 0.0900 & -0.0881 & 0.3023 \\
0.6411 & 0.0871 & 0.0864 & 0.1093 & -0.0982 & 0.3081 \\
0.5891 & 0.0952 & 0.1008 & 0.1292 & -0.1073 & 0.3138 \\
0.5370 & 0.1024 & 0.1152 & 0.1496 & -0.1151 & 0.3196 \\
0.4847 & 0.1085 & 0.1296 & 0.1705 & -0.1215 & 0.3253 \\
0.4323 & 0.1133 & 0.1440 & 0.2133 & -0.1297 & 0.3368 \\
0.3797 & 0.1164 & 0.1583 & 0.2351 & -0.1309 & 0.3426 \\
0.3271 & 0.1167 & 0.1727 & 0.2570 & -0.1303 & 0.3484 \\
0.2735 & 0.1131 & 0.1871 & 0.2787 & -0.1281 & 0.3541 \\
0.2518 & 0.1105 & 0.1929 & 0.3315 & -0.1182 & 0.3685 \\
0.2302 & 0.1073 & 0.1987 & 0.3824 & -0.1047 & 0.3829 \\
0.2087 & 0.1036 & 0.2044 & 0.4328 & -0.0897 & 0.3973 \\
0.1660 & 0.0943 & 0.2159 & 0.4831 & -0.0742 & 0.4117 \\
0.1449 & 0.0887 & 0.2217 & 0.5335 & -0.0590 & 0.4261 \\
0.1240 & 0.0823 & 0.2274 & 0.5841 & -0.0444 & 0.4405 \\
0.1033 & 0.0752 & 0.2332 & 0.6350 & -0.0309 & 0.4549 \\
0.0830 & 0.0672 & 0.2390 & 0.6862 & -0.0188 & 0.4693 \\
0.0632 & 0.0581 & 0.2447 & 0.7378 & -0.0084 & 0.4837 \\
0.0440 & 0.0477 & 0.2505 & 0.7897 & 0.0001 & 0.4981 \\
0.0258 & 0.0355 & 0.2562 & 0.8420 & 0.0061 & 0.5125 \\
0.0098 & 0.0207 & 0.2620 & 0.8946 & 0.0092 & 0.5269 \\
0.0001 & 0.0016 & 0.2677 & 0.9472 & 0.0076 & 0.5413 \\
\hline \hline
\end{tabular}




\section{APPENDIX D}

LIFT, PITCHING MOMENT, AND DRAG SUMMARY

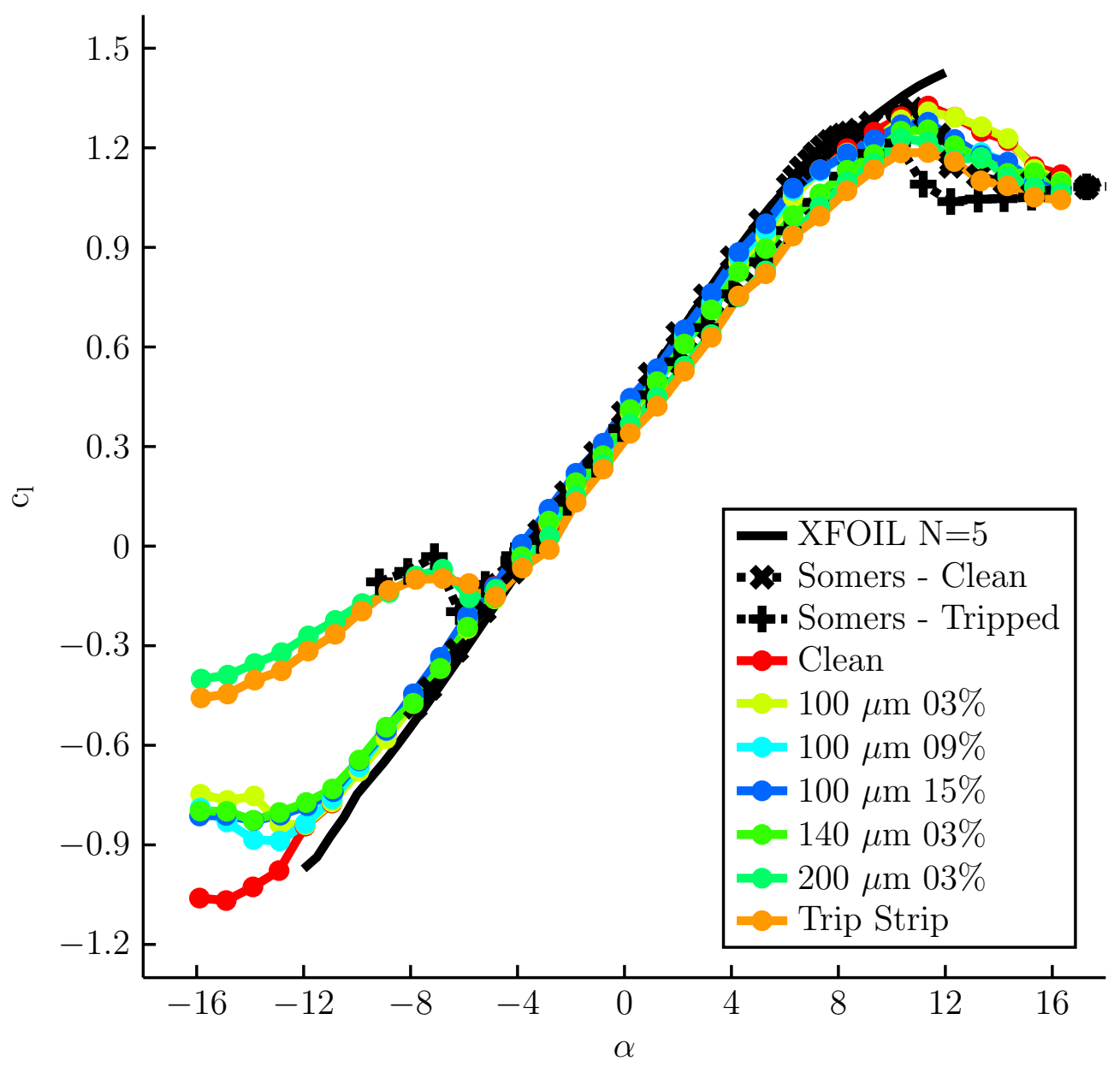

Lift variation with angle of attack for varying roughness height and density on the NREL S814 at $R e_{c}=1.6 \times 10^{6}$. 


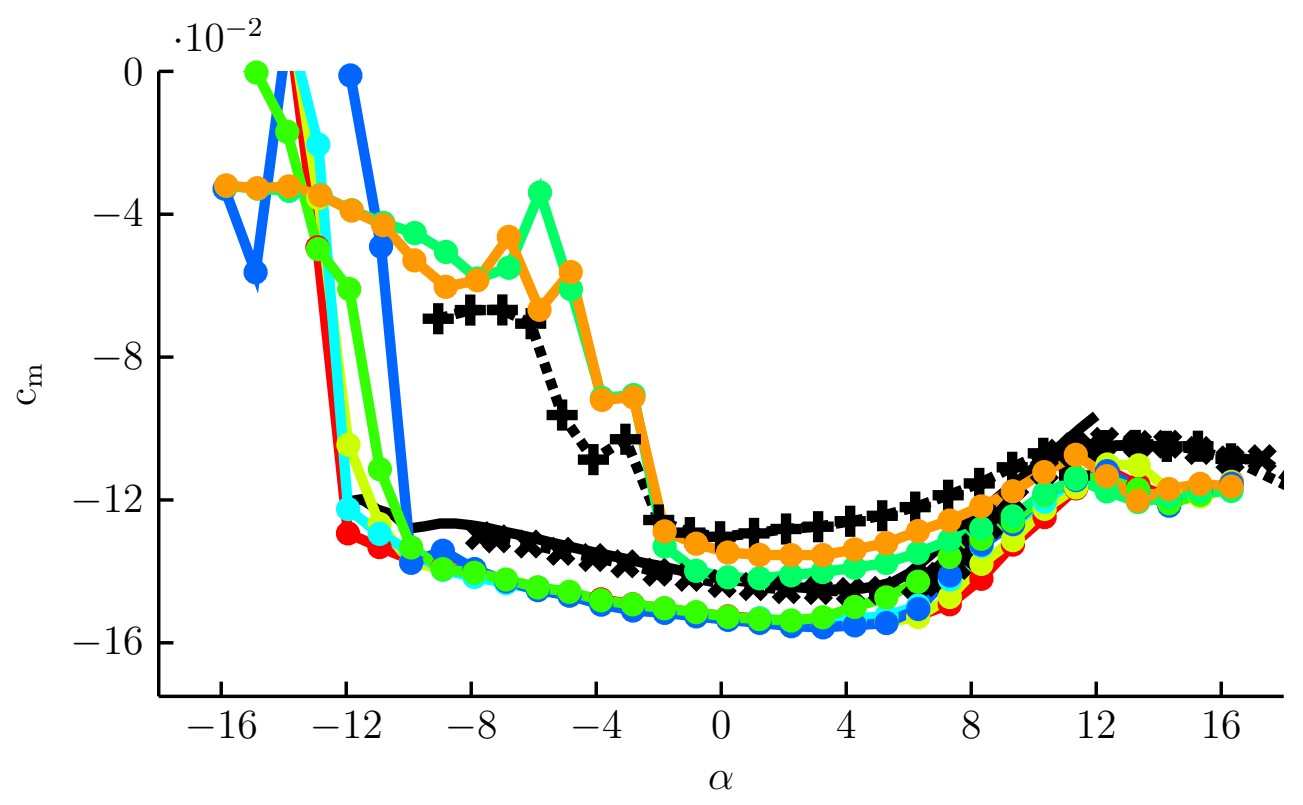

Pitching moment variation with angle of attack for varying roughness height and density on the NREL S814 at $R e_{c}=1.6 \times 10^{6}$.

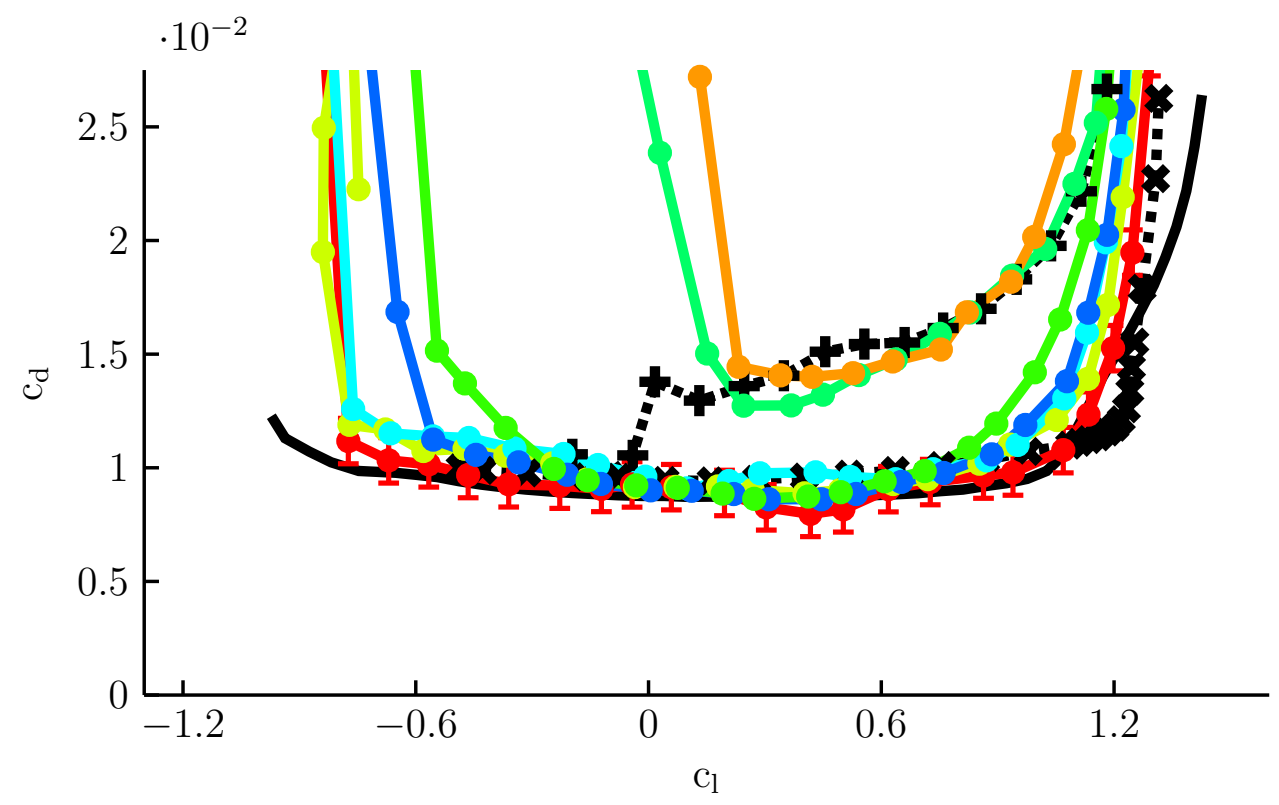

Drag variation with angle of attack for varying roughness height and density on the NREL S814 at $R e_{c}=1.6 \times 10^{6}$. 


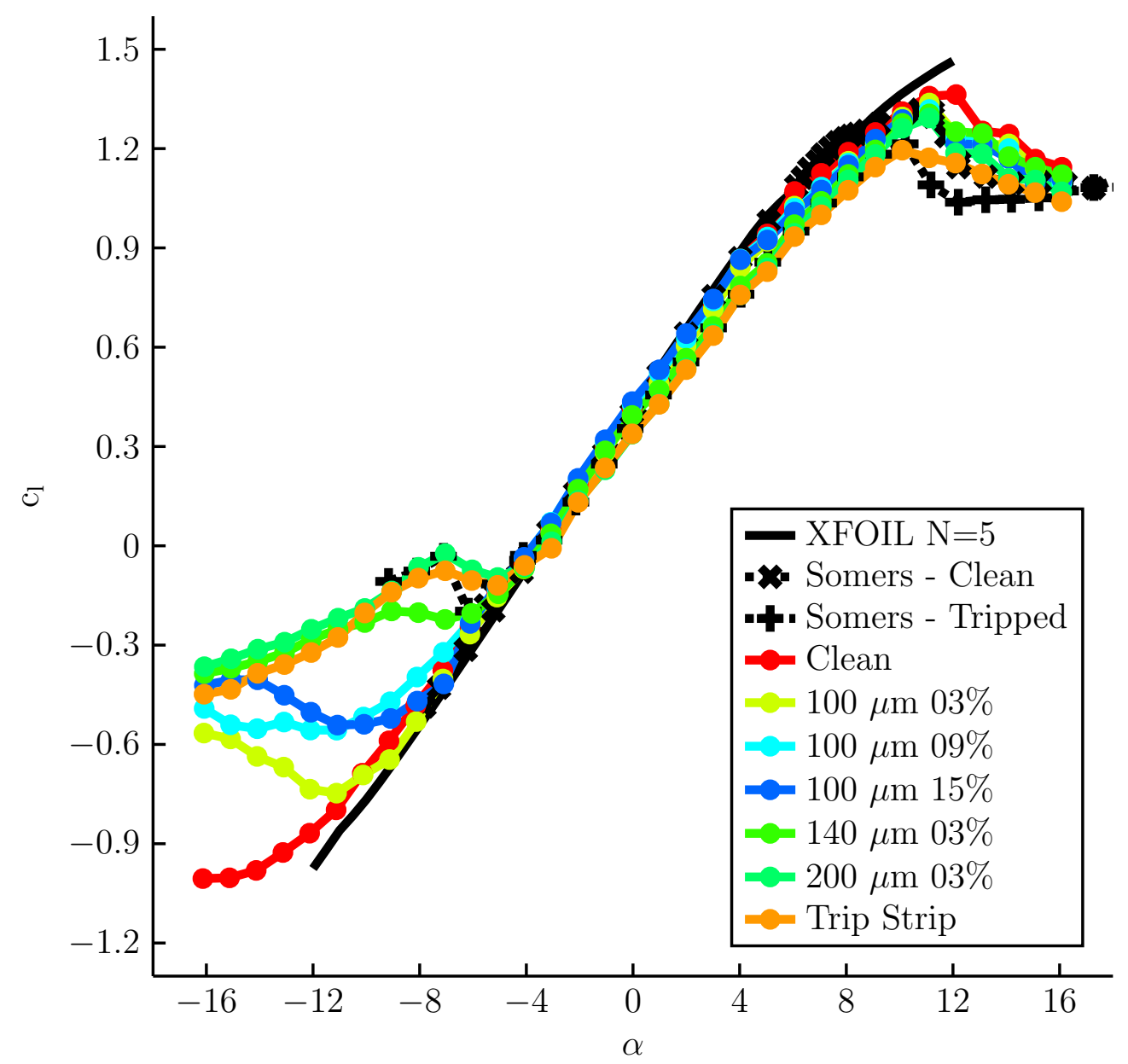

Lift variation with angle of attack for varying roughness height and density on the NREL S814 at $R e_{c}=2.4 \times 10^{6}$. 


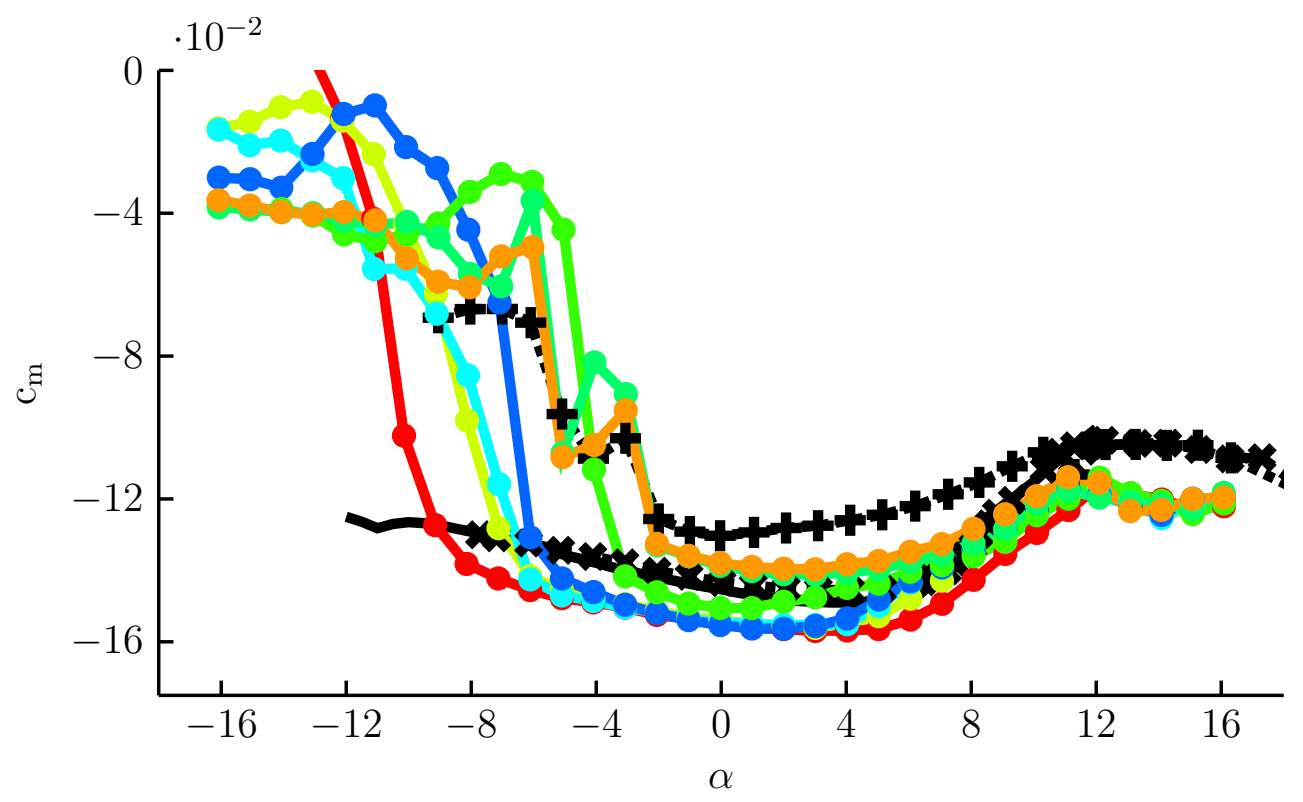

Pitching moment variation with angle of attack for varying roughness height and density on the NREL S814 at $R e_{c}=2.4 \times 10^{6}$.

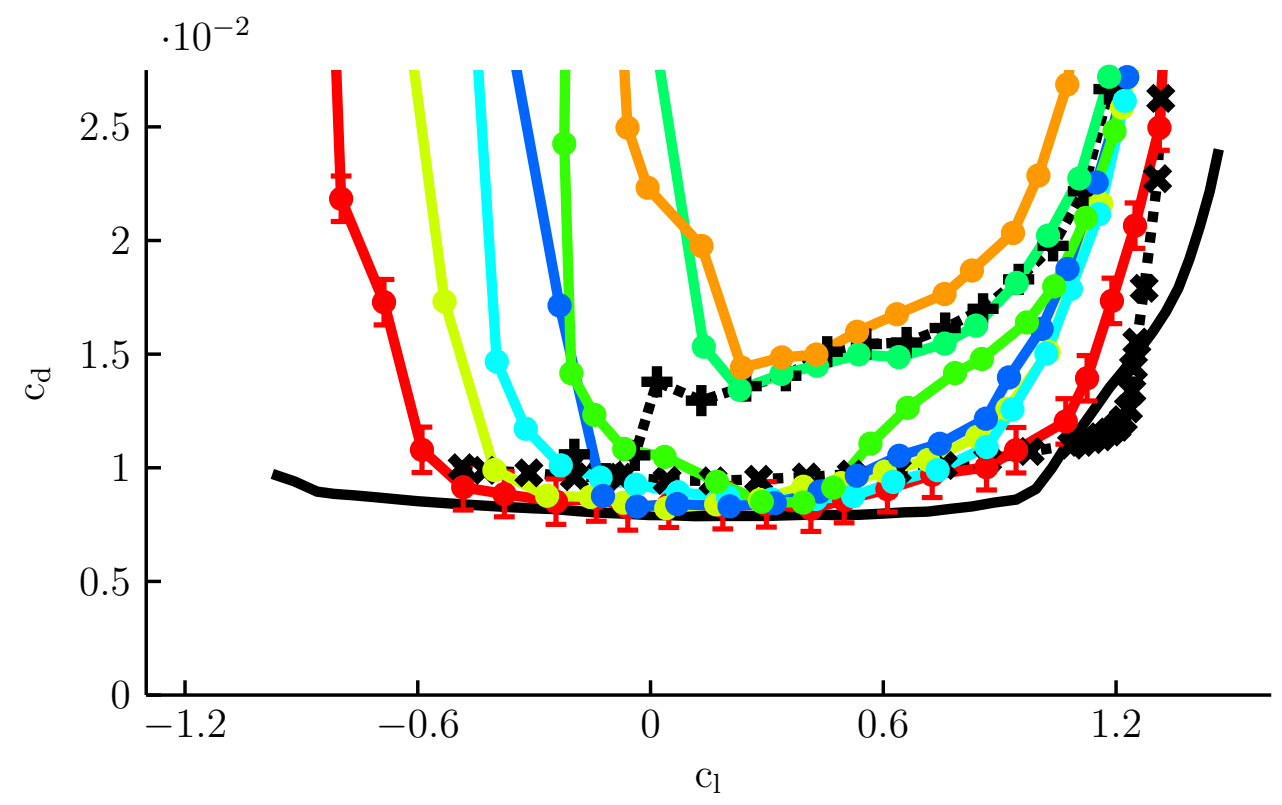

Drag variation with angle of attack for varying roughness height and density on the NREL S814 at $R e_{c}=2.4 \times 10^{6}$. 


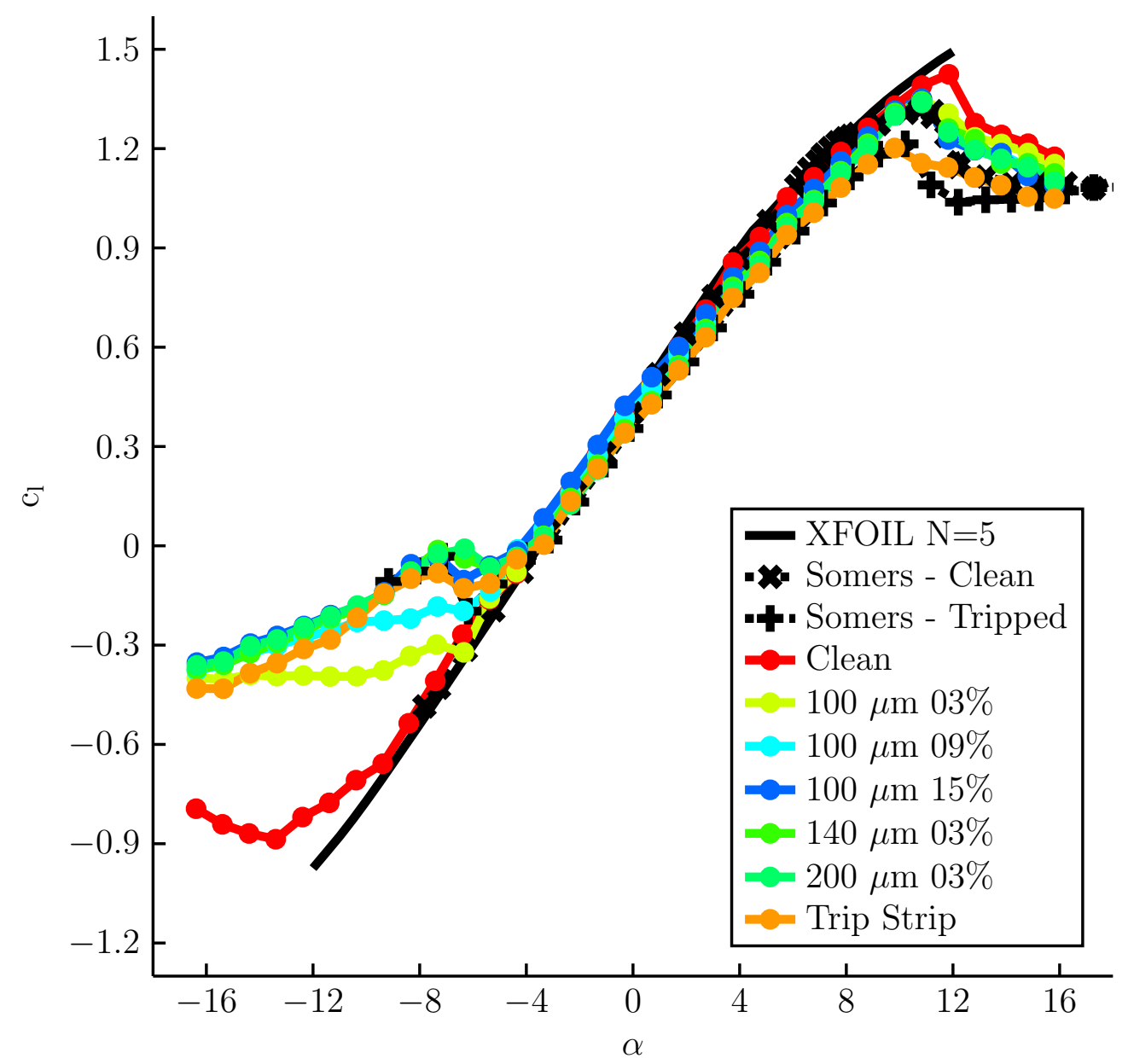

Lift variation with angle of attack for varying roughness height and density on the NREL S814 at $R e_{c}=3.2 \times 10^{6}$. 


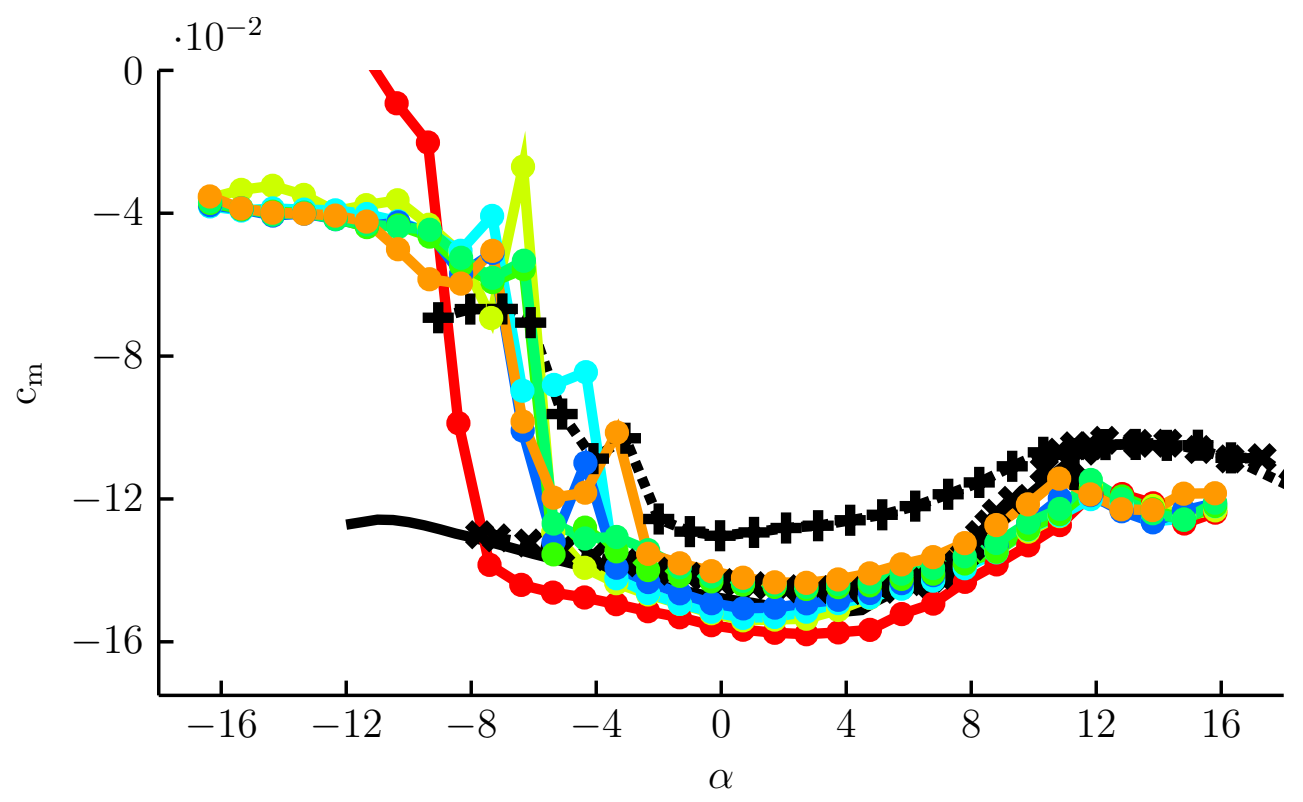

Pitching moment variation with angle of attack for varying roughness height and density on the NREL S814 at $R e_{c}=3.2 \times 10^{6}$.

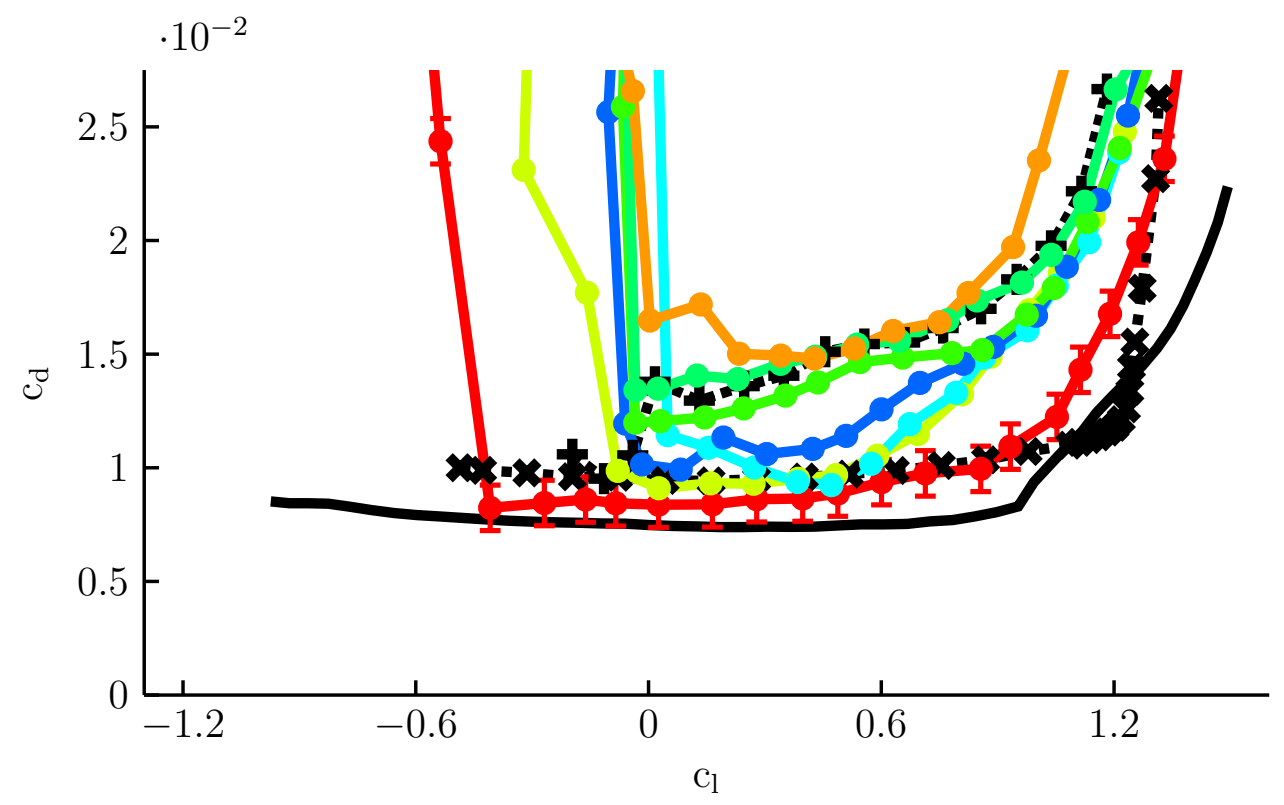

Drag variation with angle of attack for varying roughness height and density on the NREL S814 at $R e_{c}=3.2 \times 10^{6}$. 


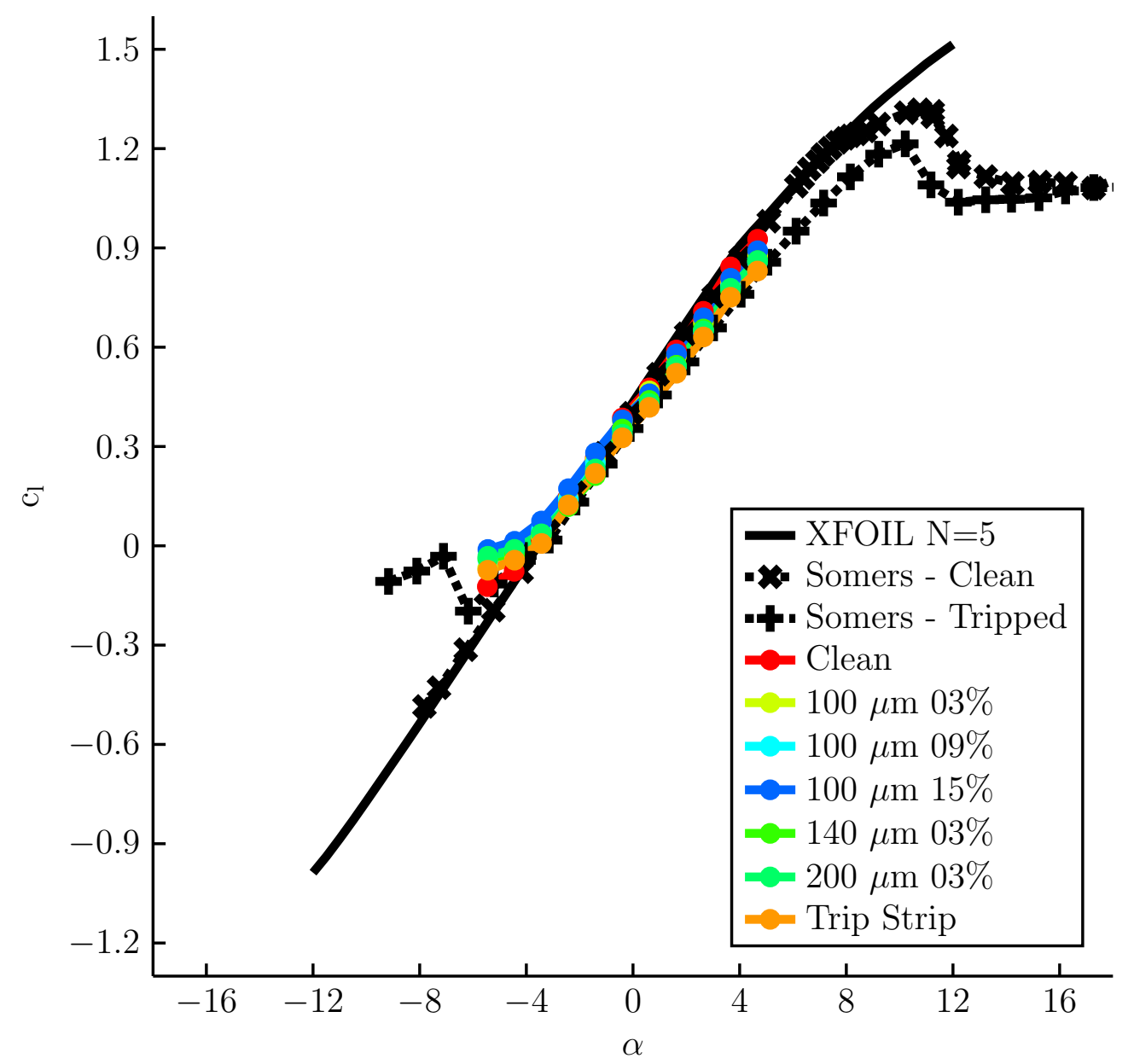

Lift variation with angle of attack for varying roughness height and density on the NREL S814 at $R e_{c}=4.0 \times 10^{6}$. 


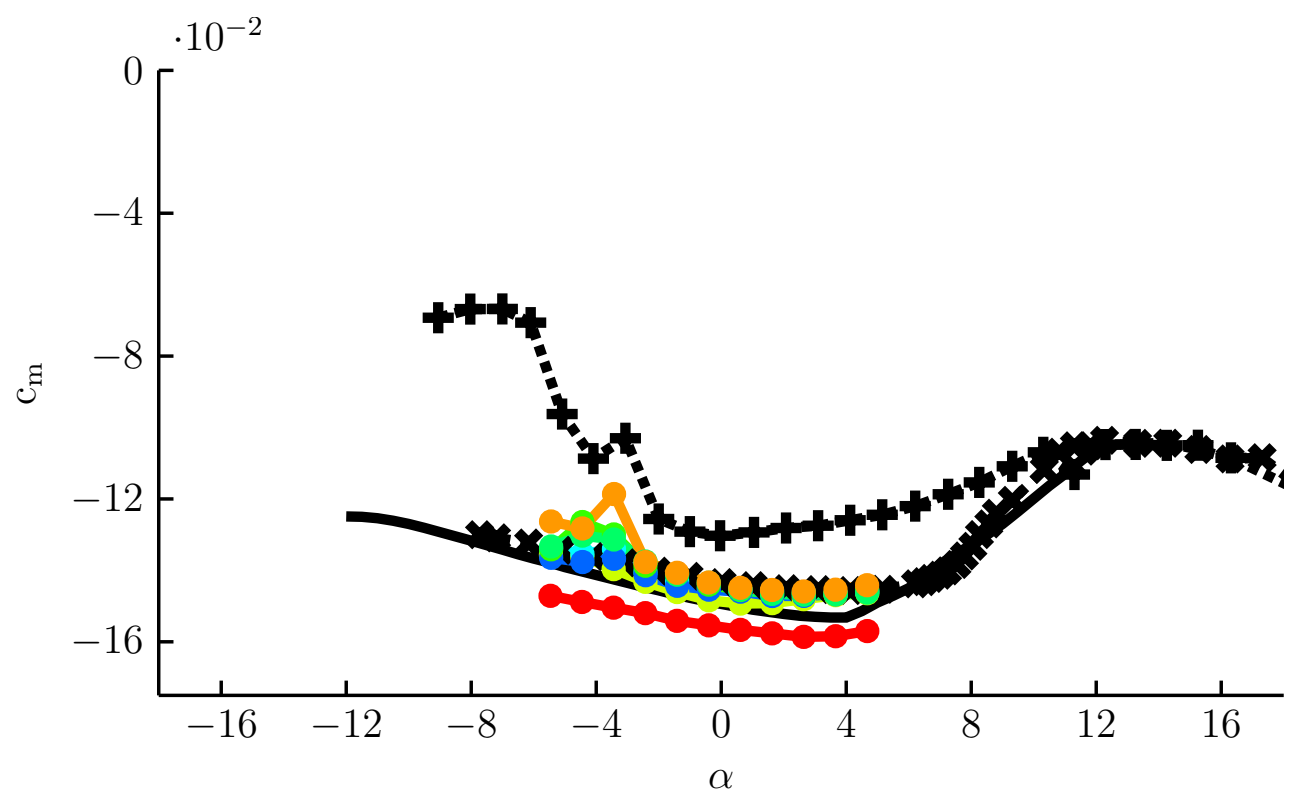

Pitching moment variation with angle of attack for varying roughness height and density on the NREL S814 at $R e_{c}=4.0 \times 10^{6}$.

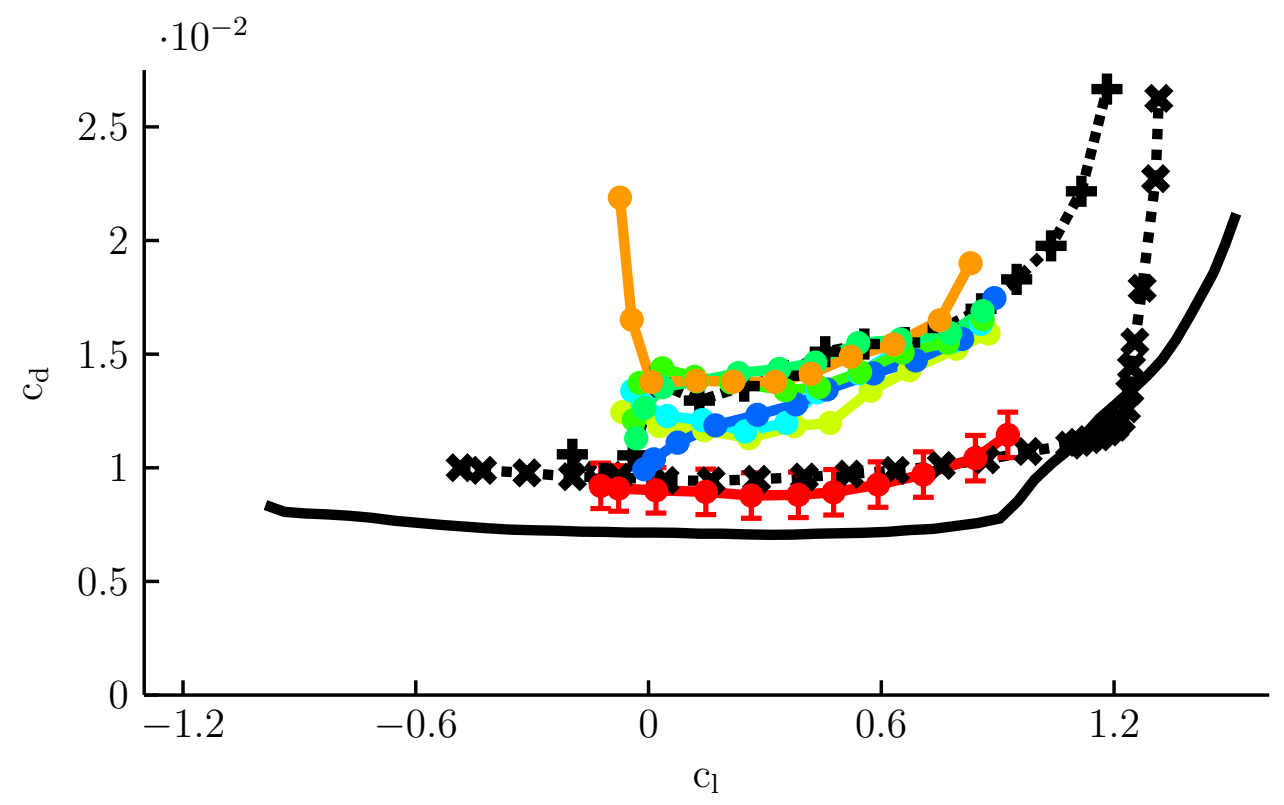

Drag variation with angle of attack for varying roughness height and density on the NREL S814 at $R e_{c}=4.0 \times 10^{6}$. 


\section{APPENDIX E}

PERFORMANCE LOSS SUMMARY

Performance losses due to roughness on NREL S814 at $R e_{c}=1.6 \times 10^{6}$.

\begin{tabular}{cccc}
\hline \hline Configuration & $d c_{l} / d \alpha$ & $c_{l, \max }$ & $\left(c_{l} / c_{d}\right)_{\max }$ \\
\hline Clean & $6.10 \mathrm{rad}^{-1}$ & 1.33 & 99.33 \\
$100 \mu \mathrm{m} \mathrm{03 \%}$ & $2.5 \%$ & $-1.3 \%$ & $-12.5 \%$ \\
$100 \mu \mathrm{m} \mathrm{09 \%}$ & $1.4 \%$ & $-3.1 \%$ & $-12.7 \%$ \\
$100 \mu \mathrm{m} \mathrm{15 \%}$ & $1.5 \%$ & $-3.4 \%$ & $-15.8 \%$ \\
$140 \mu \mathrm{m} \mathrm{03 \%}$ & $-4.0 \%$ & $-4.8 \%$ & $-23.3 \%$ \\
$200 \mu \mathrm{m} \mathrm{03 \%}$ & $-9.9 \%$ & $-6.7 \%$ & $-47.3 \%$ \\
Trip Strip & $-7.4 \%$ & $-10.2 \%$ & $-48.0 \%$ \\
\hline \hline
\end{tabular}


Performance losses due to roughness on NREL S814 at $R e_{c}=2.4 \times 10^{6}$.

\begin{tabular}{cccc}
\hline \hline Configuration & $d c_{l} / d \alpha$ & $c_{l, \max }$ & $\left(c_{l} / c_{d}\right)_{\max }$ \\
\hline Clean & $6.20 \mathrm{rad}^{-1}$ & 1.38 & 89.12 \\
$100 \mu \mathrm{m} \mathrm{03 \%}$ & $-1.5 \%$ & $-2.6 \%$ & $-16.5 \%$ \\
$100 \mu \mathrm{m} 09 \%$ & $-4.5 \%$ & $-3.9 \%$ & $-10.9 \%$ \\
$100 \mu \mathrm{m} \mathrm{15 \%}$ & $-6.9 \%$ & $-4.9 \%$ & $-20.1 \%$ \\
$140 \mu \mathrm{m} 03 \%$ & $-11.8 \%$ & $-5.0 \%$ & $-33.6 \%$ \\
$200 \mu \mathrm{m} \mathrm{03 \%}$ & $-6.8 \%$ & $-5.5 \%$ & $-41.4 \%$ \\
Trip Strip & $-8.3 \%$ & $-13.2 \%$ & $-48.4 \%$ \\
\hline \hline
\end{tabular}


Performance losses due to roughness on NREL S814 at $R e_{c}=3.2 \times 10^{6}$.

\begin{tabular}{cccc}
\hline \hline Configuration & $d c_{l} / d \alpha$ & $c_{l, \max }$ & $\left(c_{l} / c_{d}\right)_{\max }$ \\
\hline Clean & $6.26 \mathrm{rad}^{-1}$ & 1.43 & 86.74 \\
$100 \mu \mathrm{m} \mathrm{03 \%}$ & $-7.8 \%$ & $-5.9 \%$ & $-29.3 \%$ \\
$100 \mu \mathrm{m} 09 \%$ & $-9.5 \%$ & $-6.2 \%$ & $-29.8 \%$ \\
$100 \mu \mathrm{m} \mathrm{15 \%}$ & $-11.7 \%$ & $-5.3 \%$ & $-31.0 \%$ \\
$140 \mu \mathrm{m} 03 \%$ & $-5.7 \%$ & $-6.0 \%$ & $-32.4 \%$ \\
$200 \mu \mathrm{m} \mathrm{03 \%}$ & $-5.6 \%$ & $-6.4 \%$ & $-37.8 \%$ \\
Trip Strip & $-9.6 \%$ & $-16.2 \%$ & $-44.9 \%$ \\
\hline \hline
\end{tabular}




\section{APPENDIX F}

\section{TRANSITION LOCATION SUMMARY}

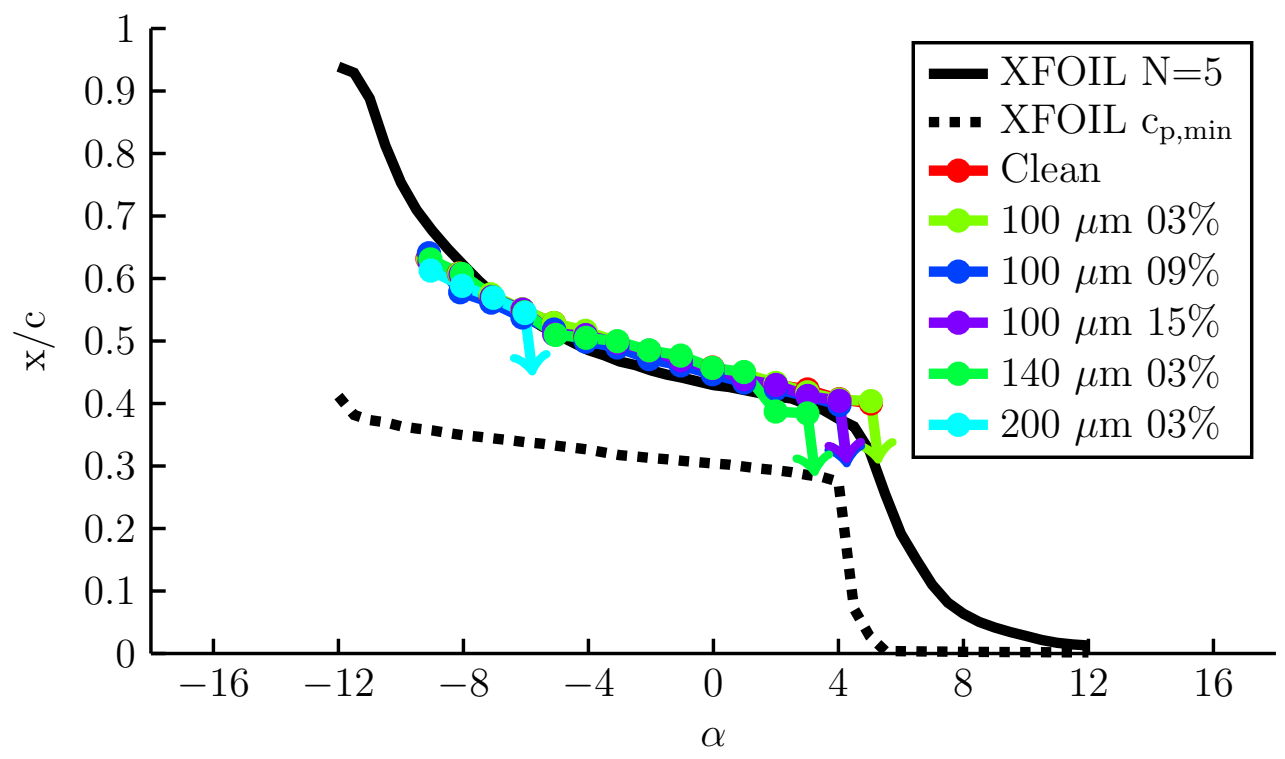

Upper surface transition location variation with angle of attack for varying roughness height and density on the NREL S814 at $R e_{c}=2.4 \times 10^{6}$. 


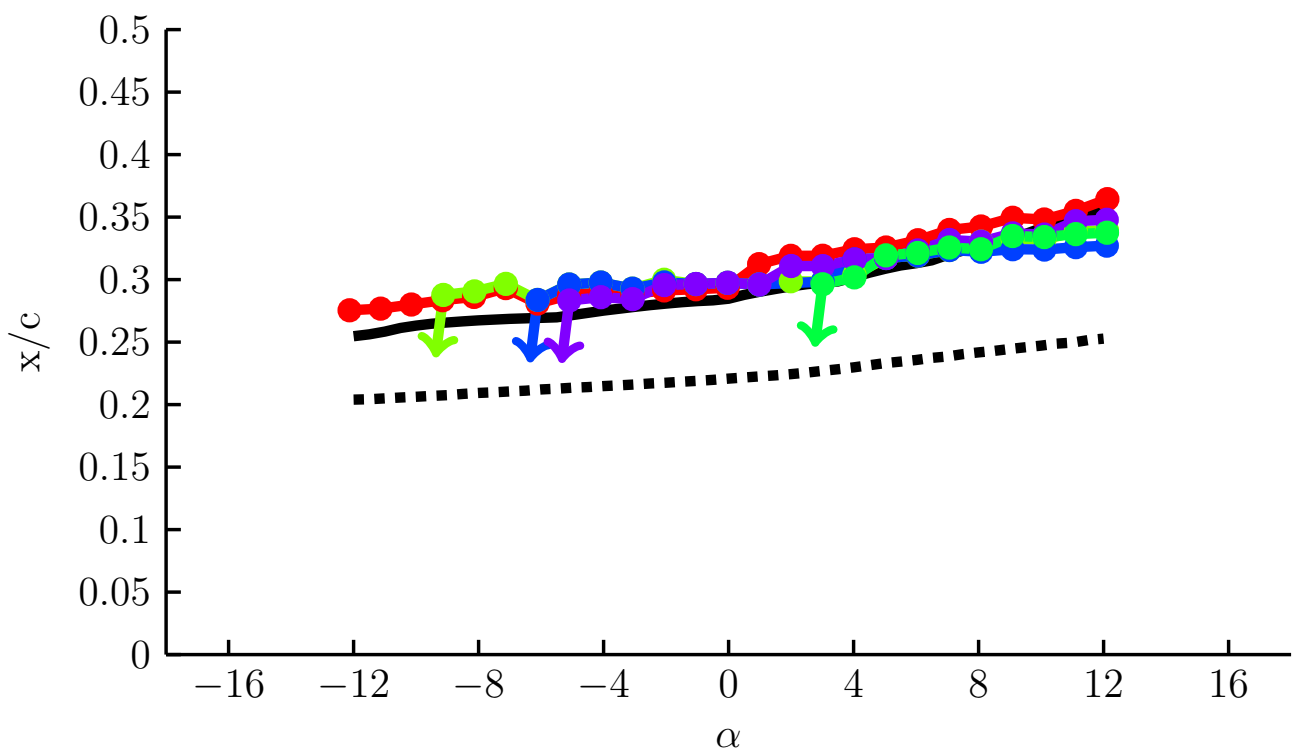

Lower surface transition location variation with angle of attack for varying roughness height and density on the NREL S814 at $R e_{c}=2.4 \times 10^{6}$. 


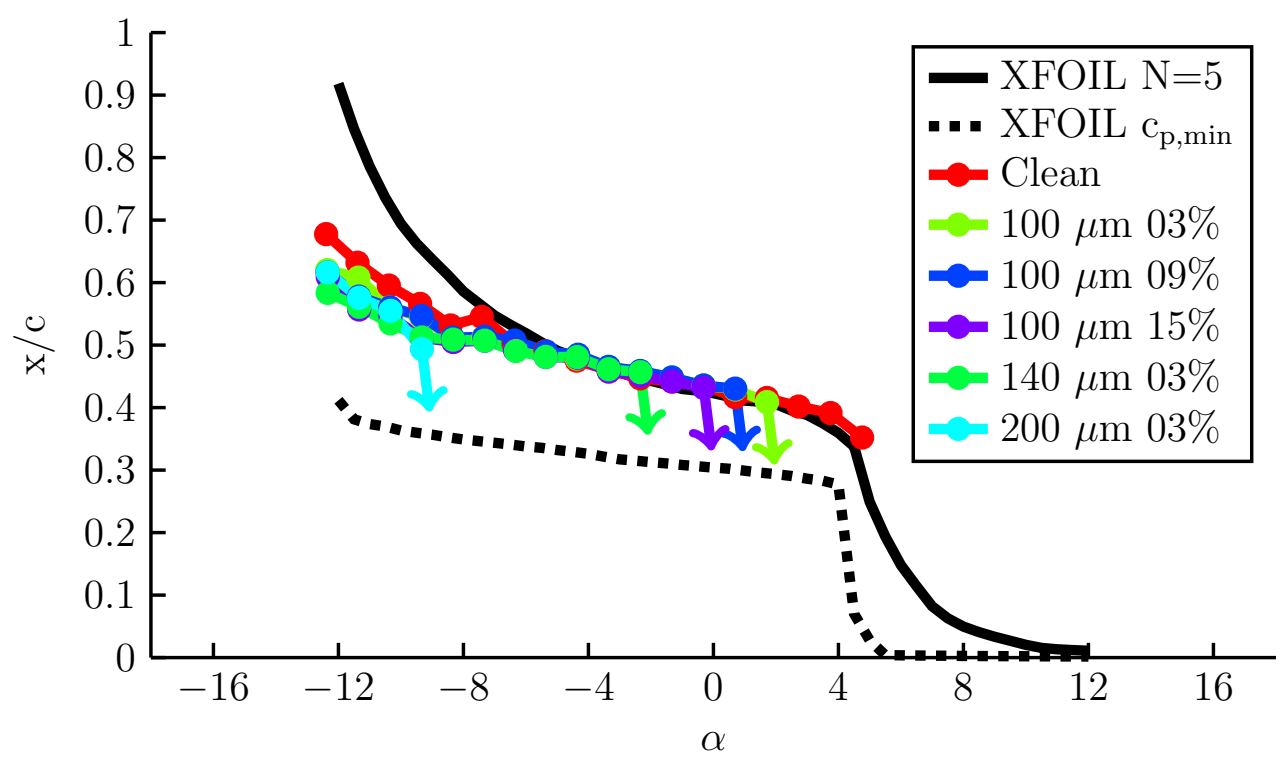

Upper surface transition location variation with angle of attack for varying roughness height and density on the NREL S814 at $R e_{c}=3.2 \times 10^{6}$. 


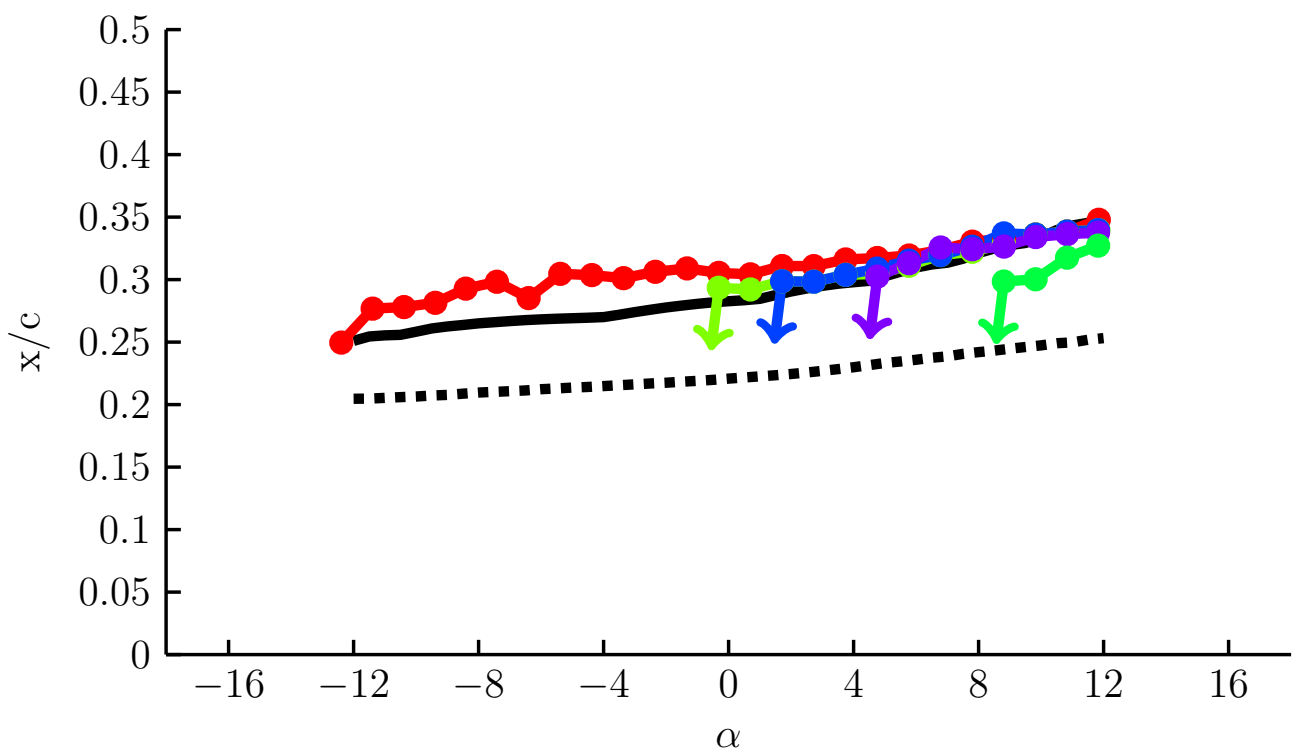

Lower surface transition location variation with angle of attack for varying roughness height and density on the NREL S814 at $R e_{c}=3.2 \times 10^{6}$. 


\section{APPENDIX G}

\section{CRITICAL REYNOLDS NUMBER SUMMARY}

Critical Reynolds number on the NREL S814 upper surface at $R e_{c}=2.4 \times 10^{6}$.

\begin{tabular}{ccc}
\hline \hline Configuration & $R e_{k, \text { crit }}$ [Experimental] & $R e_{k, \text { crit }}$ [Tani Correlation] \\
\hline $100 \mu \mathrm{m} \mathrm{03 \%}$ & $181-191$ & $168-252$ \\
$100 \mu \mathrm{m} \mathrm{09 \%}$ & $171-181$ & $168-252$ \\
$100 \mu \mathrm{m} 15 \%$ & $171-181$ & $168-252$ \\
$140 \mu \mathrm{m} \mathrm{03 \%}$ & $270-286$ & $193-289$ \\
$200 \mu \mathrm{m} \mathrm{03 \%}$ & $331-370$ & $239-358$ \\
\hline \hline
\end{tabular}

Critical Reynolds number on the NREL S814 lower surface at $R e_{c}=2.4 \times 10^{6}$.

\begin{tabular}{ccc}
\hline \hline Configuration & $R e_{k, \text { crit }}$ [Experimental] & $R e_{k, \text { crit }}$ [Tani Correlation] \\
\hline $100 \mu \mathrm{m} \mathrm{03 \%}$ & $163-164$ & $168-252$ \\
$100 \mu \mathrm{m} \mathrm{09 \%}$ & $160-162$ & $168-252$ \\
$100 \mu \mathrm{m} \mathrm{15 \%}$ & $158-160$ & $168-252$ \\
$140 \mu \mathrm{m} \mathrm{03 \%}$ & $217-226$ & $193-289$ \\
$200 \mu \mathrm{m} \mathrm{03 \%}$ & - & $239-358$ \\
\hline \hline
\end{tabular}


Critical Reynolds number on the NREL S814 upper surface at $R e_{c}=3.2 \times 10^{6}$.

\begin{tabular}{ccc}
\hline \hline Configuration & $R e_{k, \text { crit }}$ [Experimental] & $R e_{k, \text { crit }}$ [Tani Correlation] \\
\hline $100 \mu \mathrm{m} \mathrm{03 \%}$ & $227-241$ & $168-252$ \\
$100 \mu \mathrm{m} \mathrm{09 \%}$ & $214-227$ & $168-252$ \\
$100 \mu \mathrm{m} 15 \%$ & $201-214$ & $168-252$ \\
$140 \mu \mathrm{m} \mathrm{03 \%}$ & $296-313$ & $193-289$ \\
$200 \mu \mathrm{m} \mathrm{03 \%}$ & $278-342$ & $239-358$ \\
\hline \hline
\end{tabular}

Critical Reynolds number on the NREL S814 lower surface at $R e_{c}=3.2 \times 10^{6}$.

\begin{tabular}{ccc}
\hline \hline Configuration & $R e_{k, \text { crit }}[$ Experimental] & $R e_{k, \text { crit }}$ [Tani Correlation] \\
\hline $100 \mu \mathrm{m} \mathrm{03 \%}$ & $213-219$ & $168-252$ \\
$100 \mu \mathrm{m} \mathrm{09 \%}$ & $199-206$ & $168-252$ \\
$100 \mu \mathrm{m} \mathrm{15 \%}$ & $176-184$ & $168-252$ \\
$140 \mu \mathrm{m} \mathrm{03 \%}$ & $237-252$ & $193-289$ \\
$200 \mu \mathrm{m} \mathrm{03 \%}$ & - & $239-358$ \\
\hline \hline
\end{tabular}




\section{APPENDIX H}

TURBINE SIMULATION GEOMETRY

Turbine geometry for the NACA $63_{3}-418$ simulation turbine.

\begin{tabular}{cccc}
\hline \hline $\mathrm{r}[\mathrm{m}]$ & Airfoil Name & Twist $\left[^{\circ}\right]$ & Chord $[\mathrm{m}]$ \\
\hline 0.00 & Cylinder1 & 13.31 & 3.00 \\
6.30 & Cylinder1 & 13.31 & 3.75 \\
7.88 & Cylinder2 & 13.31 & 4.13 \\
12.60 & NACA 63 $33^{-418}$ & 13.31 & 4.50 \\
31.50 & NACA 63 $33^{-418}$ & 6.54 & 3.38 \\
34.65 & NACA 63 $33^{-418}$ & 5.36 & 3.19 \\
63.00 & NACA 63 $33^{-418}$ & 0.00 & 1.50 \\
\hline \hline
\end{tabular}


Turbine geometry for the NREL S814 simulation turbine.

\begin{tabular}{cccc}
\hline \hline $\mathrm{r}[\mathrm{m}]$ & Airfoil Name & Twist [] & Chord [m] \\
\hline 0.00 & Cylinder1 & 13.31 & 3.00 \\
6.30 & Cylinder1 & 13.31 & 3.75 \\
7.88 & Cylinder2 & 13.31 & 4.13 \\
12.60 & NREL S814 & 13.31 & 4.50 \\
31.50 & NREL S814 & 6.54 & 3.38 \\
34.65 & NREL S814 & 5.36 & 3.19 \\
63.00 & NREL S814 & 0.00 & 1.50 \\
\hline \hline
\end{tabular}


Turbine geometry for the combined NACA $63_{3}-418$ and NREL S814 simulation turbine.

\begin{tabular}{cccc}
\hline \hline $\mathrm{r}[\mathrm{m}]$ & Airfoil Name & ${\text { Twist }\left[{ }^{\circ}\right]}$ & Chord $[\mathrm{m}]$ \\
\hline 0.00 & Cylinder1 & 13.31 & 3.00 \\
6.30 & Cylinder1 & 13.31 & 3.75 \\
7.88 & Cylinder2 & 13.31 & 4.13 \\
12.60 & NREL S814 & 13.31 & 4.50 \\
31.50 & NREL S814 & 6.54 & 3.38 \\
34.65 & NACA $63_{3}-418$ & 5.36 & 3.19 \\
63.00 & NACA $63_{3}-418$ & 0.00 & 1.50 \\
\hline \hline
\end{tabular}

